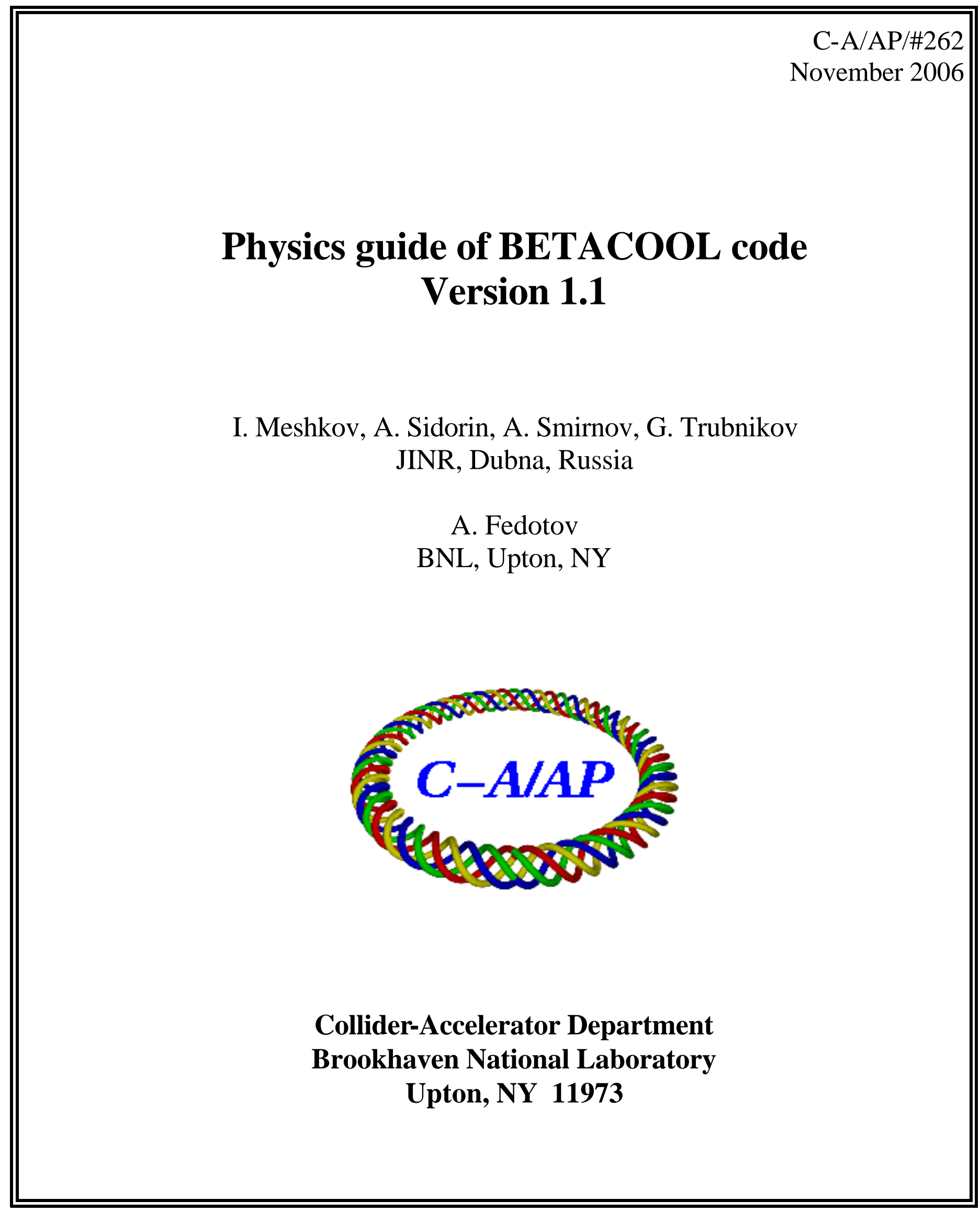




\title{
Physics guide of BETACOOL code Version 1.1
}

\author{
I. Meshkov, A. Sidorin, A. Smirnov, G. Trubnikov \\ JINR, Dubna, Russia
}

A. Fedotov

BNL, Upton, NY, USA

General goal of the BETACOOL program is to simulate long term processes (in comparison with the ion revolution period) leading to variation of the ion distribution function in 6 dimensional phase space. The ion beam motion inside a storage ring is assumed to be stable and it is treated in linear approximation.

BETACOOL code was developed in collaboration with many scientific centers:

BNL, Upton, USA

Fermilab, Batavia, USA

Tech-X, Boulder, USA

RIKEN, Wako, Japan

NIRS, Chiba, Japan

Kyoto Univ., Japan

CERN, Geneva, Switzerland

GSI, Darmstadt, Germany

FZJ, Juelich, Germany

Erlangen Univ., Germany

Munich Univ., Germany

ITEP, Moscow, Russia

BINP, Novosibirsk, Russia

Uppsala Univ., Sweden

Stockholm Univ., Sweden

In general, ion beam dynamics within BETACOOL can be simulated taking into account various effects, such as Electron Cooling, Intrabeam Scattering, Rest Gas Scattering, Stochastic Cooling, Colliding Beams effects, Recombination, Target Scattering and others. Depending on the task, one can use an arbitrary combination of these effects in simulations.

The Physics guide described in this report summarizes only two major effects of Electron Cooling and Intrabeam Scattering which are typically used in simulations of electron cooling. The description is given as three parts with independent references to sections and equations within each of the parts. This guide includes the following parts:

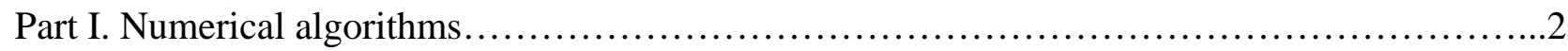

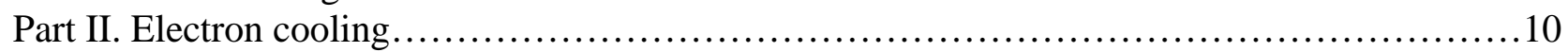

Part III. Intrabeam scattering ...................................................... 44 


\section{Part I. Numerical algorithms}

\section{Introduction}

Initially, BETACOOL [1] code was developed as a program for simulation of particle dynamics in ion storage rings under the action of electron cooling force. Further development led to addition of other effects and numerical algorithms for tracking.

First algorithm of numerical tracking is RMS Dynamics which calculates evolution in time of the r.m.s. (root mean square) parameters of the ion beam distribution function and particle number. RMS Dynamics algorithm is based on solution of equation for the second order moments of the distribution function. Characteristic times of the evolution of the beam parameters for a few general effects are calculated under assumption of Gaussian shape of the distribution function.

Second algorithm of tracking is Model Beam approach. It was developed on the basis of SIMCOOL code which was originally written by BINP group at Novosibirsk. This algorithm uses a few thousands of test particles with an arbitrary distribution. The action from IBS on each of the test particles is calculated from the instantaneous distribution of the test particles. This algorithm has a good accuracy when the distribution of test particles is close to Gaussian. Some modification of this method was made for simulation of IBS in the case of non Gaussian distribution.

Evolution of the ion distribution function is described by the Fokker-Plank equation. In general case, the friction and diffusion terms depend on the distribution function. However, in some cases when the effects acting on the distribution function do not lead to change of its shape, the FokkerPlank equation can be reduced to equation for the second order moments of the distribution function.

In a general case, the Fokker-Plank equation can be reduced to the Langevin equation in invariant or momentum space. The Model Beam algorithm realizes solution of the Langevin equation in momentum space using Monte Carlo method. In the framework of this algorithm the ion beam is presented as a particle array. Each particle is presented as a 6 co-ordinate vector: $\vec{X}=\left(x, \frac{p_{x}}{p}, y, \frac{p_{y}}{p}, s-s_{0}, \frac{\Delta p}{p}\right)$, where $x$ and $y$ are the horizontal and vertical co-ordinates, $p_{x}$ and $p_{y}$ are corresponding momentum components, $s-s_{0}$ is the distance from the bunch center (in the case of coasting beam - distance from a reference particle), $\Delta p$ is the particle momentum deviation from momentum of reference particle $p$. Action of each of the effects is simulated as variation of the particle momentum according to:

$$
\left(p_{x, y, s} / p\right)_{f i n}=\left(p_{x, y, s} / p\right)_{i n}+\Lambda_{x, y, s} \Delta T+\sqrt{D_{x, y, s} \Delta T \xi_{x, y, s}},
$$

where $p_{s}$ is the particle longitudinal momentum deviation, subscript in corresponds to initial momentum value, subscript fin corresponds to final particle momentum after action of the effect, $\Lambda$ and $D$ are the drift and diffusion terms for the corresponding degree of freedom, $\Delta T$ is a step of the integration over time, $\xi$ is a random number for Gaussian distribution with unit dispersion.

The third algorithm, which is called MD Tracking, uses real particles with arbitrary distribution. This special method, named Molecular Dynamics (MD) [2], is used for simulation of Intrabeam Scattering (IBS) in the ion beam. This algorithm assumes that particles have periodical distribution in the longitudinal direction. Usual number of particles per cell is about 10-100. It can simulate the 
crystalline state of ion beam for very low temperature in the beam rest frame. MD simulation can be used for testing of analytical formulas for IBS effect also. However, it presently works only for unbunched ion beam. Therefore, we do not describe this algorithm in this report.

Three different tracking algorithms described above allow to benchmark them against each other and to test the validity of action due to different physical effects. If initially the Gaussian distribution is chosen then the evolution of r.m.s. parameters should be the same while the shape of the distribution doesn't change.

\section{RMS Dynamics algorithm}

\subsection{Physical model}

The physical model which can be investigated with this algorithm is based on the following general assumptions:

1) ion beam has Gaussian distribution over all degrees of freedom, and is not changed during the process.

2) algorithm for analysis of the problem is considered as a solution of the equations for r.m.s. values of the beam phase space volumes of three degrees of freedom.

3) maxima of all the distribution functions coincide with equilibrium orbit.

The evolution of the ion beam parameters during its motion inside the storage ring is described by the following system of four differential equations:

$$
\left\{\begin{array}{l}
\frac{\varepsilon_{\text {hor }}}{d t}=\varepsilon_{\text {hor }} \sum_{j} \frac{1}{\tau_{\text {hor }}}, \\
\frac{\varepsilon_{v e r}}{d t}=\varepsilon_{\text {ver }} \sum_{j} \frac{1}{\tau_{v e r}}, \\
\frac{\varepsilon_{\text {lon }}}{d t}=\varepsilon_{\text {lon }} \sum_{j} \frac{1}{\tau_{\text {lon }}}, \\
\frac{d N}{d t}=N \sum_{j} \frac{1}{\tau_{\text {life }}},
\end{array}\right.
$$

where $N$ is the number of particles. For the transverse degrees of freedom parameters $\varepsilon_{h o r}$ and $\varepsilon_{v e r}$ correspond to the horizontal and vertical emittances, for the longitudinal degree of freedom it is given by the following expression:

$$
\varepsilon_{\text {lon }}= \begin{cases}\left(\frac{\Delta p}{p}\right)^{2}, & \text { coasting beam } \\ \left(\frac{\Delta p}{p}\right)^{2}+\frac{1}{\Omega_{s}{ }^{2}}\left[\frac{d}{d t}\left(\frac{\Delta p}{p}\right)\right]^{2}, & \text { bunched beam } .\end{cases}
$$

In Eq. (1.3) the upper line corresponds to a coasting beam, lower line to a bunched beam with constant parameters (for variable synchrotron frequency it is necessary to use adiabatic invariant instead of energy; presently a depression of the synchrotron tune due to the action of the beam 
space charge is not taken into account during dynamics simulation). $\Omega_{\mathrm{s}}-$ is the synchrotron frequency. Therefore, the second expression in Eq. (1.3) corresponds to the square of momentum oscillation amplitude.

Characteristic times $\left(\tau_{h o r}, \tau_{v e r}, \tau_{\text {lon }}\right)$ are functions of all three emittances and particle number, and have positive sign for a heating process and negative for cooling. The negative sign of the lifetime $\left(\tau_{\text {life }}\right.$ ) corresponds to the particle loss. The sign of the lifetime can be positive in the presence of particle injection, when particle number increases.

Index $j$ in Eq. (1.2) is the number of processes (effects) involved in the calculations. The program structure is designed in a way which allows to include any effect into calculation, if the effect can be described by cooling or heating rates.

During numerical solution of the system (1.2) the parameters, which characterize beam stability are also calculated. They are the incoherent betatron tune shift value, the depression of the synchrotron tune, dimensionless parameters describing the beam in the framework of the longitudinal and transverse coherent instabilities.

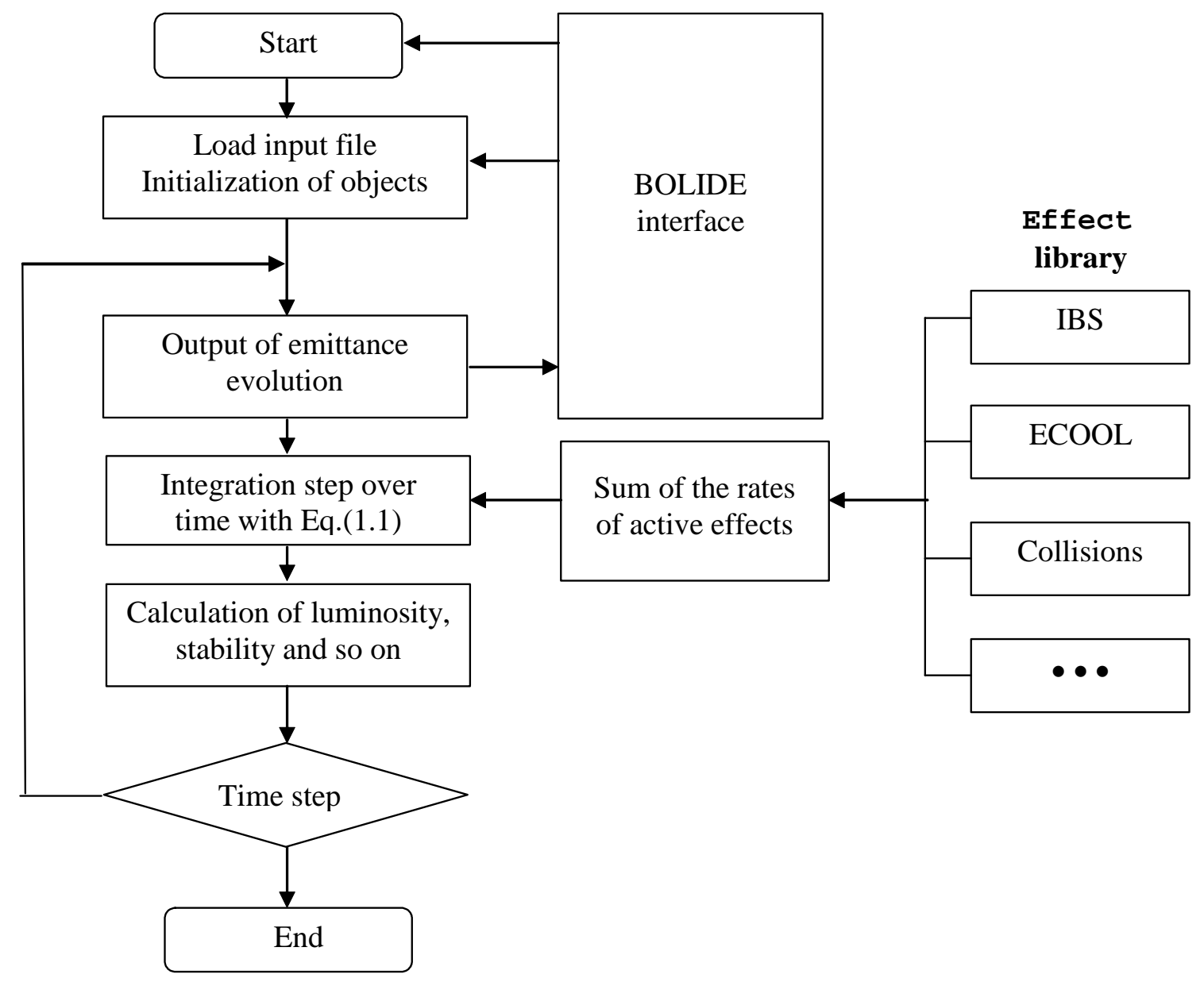

Fig. 1.1 Block-scheme of the r.m.s. beam parameter evolution simulation

Numerical solution of the system (1.2) is performed using Euler method with automatic step variation. In principle, the system can be solved using arbitrary method - for example, one of the Runge-Kutta methods. Choice of the procedure for integration is determined by optimization of the calculation speed. Algorithm of numerical integration of the system of equations (1.2) is realized in the program as illustrated in the Fig. 1.1. 
Further development of the code is related to the modifications of this basic model. Such improvement is necessary due to several disadvantages of the model. For example, an assumption of Gaussian shape of the ion distribution function. This assumption is more or less realistic in an equilibrium state of the ion beam when the equilibrium is determined by many processes of stochastic nature. If the equilibrium does not exist due to a fast loss of particles or during initial stage of the beam cooling, the ion distribution function can be far from Gaussian. The same situation takes place in an experiment with internal targets which dimensions are not coinciding with the ion beam dimensions. The ionization energy losses of the ion beam in the target can not be correctly calculated in the framework of existing model also.

Investigation of the ion beam dynamics for an arbitrary shape of the distribution function can be performed using multi particle simulation. The structure of basic objects of the code, such as the models of the ion ring and the ion beam, are developed in a way which allows to realize the multi particle simulation using Monte Carlo method without any change of the program structure.

\section{Model Beam algorithm}

The Model Beam algorithm was borrowed from the SIMCOOL code. Implementation of this algorithm was very useful for the benchmarking of several numerical models between the codes. This algorithm was further developed within the BETACOOL code.

\subsection{Physical model}

Investigation of ion beam dynamics for an arbitrary distribution function is performed using multi particle simulation in the framework of Model Beam algorithm. In this algorithm the ion beam is represented by an array of modeled particles. The heating and cooling processes involved in simulations result in a change of the particle momentum components and number of particles, which are calculated in accordance with the time step of dynamic simulation.

Each effect is located in some position of the ring characterized by the ring lattice functions. Transformation of the beam inside the ring is provided using linear matrix with random phase advance between the locations of the effects.

The numerical realization of the algorithm described by the formula (1.1) has the following peculiarity. The regular variation of the particle momentum due to action of drift term can be rewritten as

$$
\left(p_{x, y, s} / p\right)_{f i n}=\left(p_{x, y, s} / p\right)_{i n}\left(1+\frac{\Lambda_{x, y, s}}{\left(p_{x, y, s} / p\right)_{i n}} \Delta T\right)
$$

For large value of $\Delta T$ the absolute value of the term $\frac{\Lambda_{x, y, s}}{\left(p_{x, y, s} / p\right)_{i n}} \Delta T$ can be larger than unity (in the case of cooling this term has a negative sign). In this case direct application of the formula (2.1) will lead to change in sign of corresponding momentum component and can lead also to increase of its absolute value. This situation corresponds to artificial diffusion heating of the beam due to numerical algorithm. To avoid this "numerical" diffusion for $\left|\frac{\Lambda_{x, y, s}}{\left(p_{x, y, s} / p\right)_{i n}} \Delta T\right|>1$ the formula (2.1) is transformed to the following form 


$$
\left(p_{x, y, s} / p\right)_{f i n}=\left(p_{x, y, s} / p\right)_{i n} \times \exp \left\{\frac{\Lambda_{x, y, s}}{\left(p_{x, y, s} / p\right)_{i n}} \Delta T\right\},
$$

which includes the (2.1) as a limiting case for small $\Delta T$.

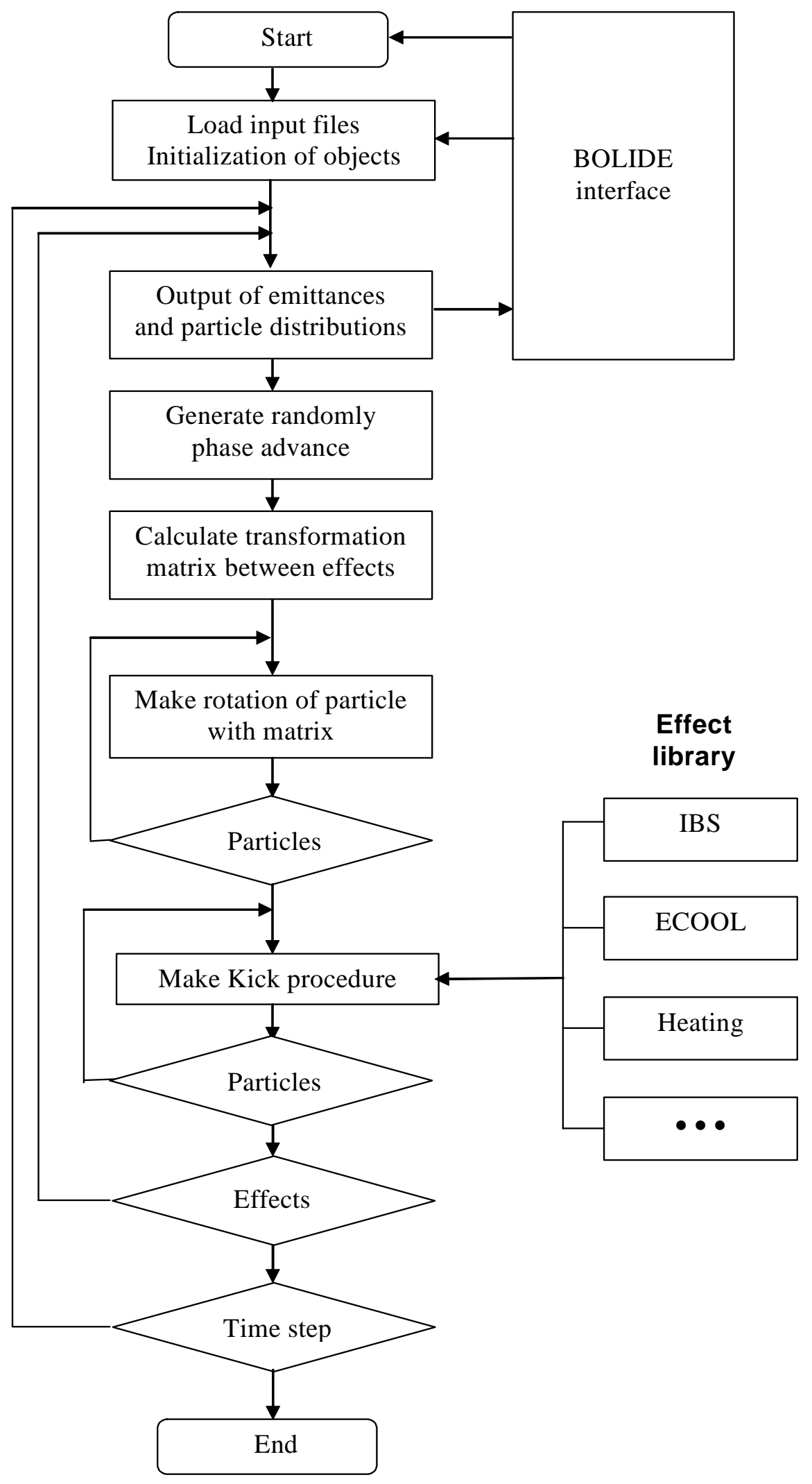

Fig. 2.1. Block scheme of the Model Beam algorithm. 
The Model Beam algorithm can be illustrated by the scheme presented in the Fig. 2.1, includes the following steps:

- initially the storage ring optic structure is loaded from external file (MAD output file, for example), mean ring parameters and RF system parameters are loaded from input BETACOOL file, - in the initial position of the ring an array of particles is generated using random number generator and matched with the ring lattice functions and synchrotron function,

- on each cycle over the effects, the transformation matrix between two effects is calculated from its lattice functions, phase advance is generated randomly,

- each particle is rotated according to the transformation matrix,

- kick procedure from the current effect is applied to each particle.

\subsection{The beam rotation with the matrix}

To avoid the problems related to the beam mismatching and coupling between longitudinal and transverse coordinates the beam rotation in the ring is provided in the following steps.

The betatron coordinates are transformed according to the coefficients of the ring matrix in the point of the array generation:

$$
\left(\begin{array}{c}
x \\
x^{\prime}
\end{array}\right)_{i+1}=\left(\begin{array}{ll}
R_{11} & R_{12} \\
R_{21} & R_{22}
\end{array}\right)\left(\begin{array}{c}
x \\
x^{\prime}
\end{array}\right)_{i},\left(\begin{array}{c}
y \\
y^{\prime}
\end{array}\right)_{i+1}=\left(\begin{array}{ll}
R_{33} & R_{34} \\
R_{43} & R_{44}
\end{array}\right)\left(\begin{array}{c}
y \\
y^{\prime}
\end{array}\right)_{i},
$$

where notation of the matrix coefficients is explained in the following table:

$$
M_{R I N G}=\left(\begin{array}{llllll}
R_{11} & R_{12} & R_{13} & R_{14} & R_{15} & R_{16} \\
R_{21} & R_{22} & R_{23} & R_{24} & R_{25} & R_{26} \\
R_{31} & R_{32} & R_{33} & R_{34} & R_{35} & R_{36} \\
R_{41} & R_{42} & R_{43} & R_{44} & R_{45} & R_{46} \\
R_{51} & R_{52} & R_{53} & R_{54} & R_{55} & R_{56} \\
R_{61} & R_{62} & R_{63} & R_{64} & R_{65} & R_{66}
\end{array}\right) .
$$

The beam rotation in the longitudinal plane is provided with the usual rotation matrix:

$$
\left(\begin{array}{c}
s-s_{0} \\
\frac{\Delta p}{p}
\end{array}\right)_{i+1}=\left(\begin{array}{cc}
\cos \mu & B_{s} \sin \mu \\
-\frac{1}{B_{s}} \sin \mu & \cos \mu
\end{array}\right)\left(\begin{array}{c}
s-s_{0} \\
\frac{\Delta p}{p}
\end{array}\right)_{i}
$$

where $\mu$ value can be arbitrary non-zero, $B_{s}$ - synchrotron function.

After the beam rotation in the longitudinal plane new values of the particles transverse co-ordinates are calculated from the betatron ones and new value of the longitudinal momentum deviation. 


\subsection{Kick procedure for IBS}

In the case of IBS calculation based on mean growth rates, the mean growth rates are calculated in accordance with one of the analytical models implemented in BETACOOL and the ring structure loaded from output MAD file. When the growth rates are known one can calculate mean square of the scattering angle taking into account multiplication factor. The mean square angle after one revolution in the ring is equal:

$$
\left\langle\theta^{2}\right\rangle=\frac{\varepsilon}{\beta} \frac{T_{r e v}}{\tau},
$$

where $\beta$ is the beta function in the point of the particle array generation, $\tau$ is characteristic growth time for the corresponding degree of freedom. Here, the angular deviation of the particle trajectory means relative momentum components: $\theta_{x, y}=\frac{p_{x, y}}{p}, \theta_{s}=\frac{\Delta p}{p}$. After $N_{t u r n}$ revolutions in the ring the square of the scattering angle is equal to the sum of the square angles of each revolution:

$$
\left\langle\theta^{2}\right\rangle=\frac{\varepsilon}{\beta} \frac{T_{r e v}}{\tau} N_{\text {turn }}
$$

The variation of the particle trajectory angular deviation is calculated according to

$$
\Delta \theta=\sqrt{\left\langle\theta^{2}\right\rangle} \cdot \xi
$$

where $\xi$ is the random value with Gaussian distribution of unit dispersion.

\subsection{Kick procedure for Electron Cooling (ECOOL)}

In the framework of Model Beam algorithm the action of the electron cooling on the ion momentum components is calculated in accordance with the cooler representation as a thin lens. In this case the ion angle variation is calculated as

$$
\Delta \theta=\frac{F}{M c^{2} \beta^{2} \gamma} l_{\text {cool }} N_{\text {turn }}
$$

where $F$ is the friction force in laboratory rest frame (LRF), $M$ is the ion mass, $l_{\text {cool }}$ is the cooling section length, $N_{\text {turn }}$ is the number of revolutions used as a multiplication factor. The ion coordinates change is neglected inside the cooler. The friction force components can be calculated using any of the friction force formulae and any of the models of electron beam implemented in the BETACOOL.

\subsection{Kick procedure for effect of Additional Heating}

An effect of Additional Heating can be applied for different kind of kicks. An angle variation in the case of constant and linear heating (or cooling) is calculated with formula: 


$$
\Delta \theta_{i}=\theta_{i} \times\left\{\begin{array}{cc}
\frac{b}{\varepsilon_{i}} \cdot \frac{T_{r e v}}{\tau_{i}} N_{\text {turn }}, & \frac{b}{\varepsilon_{i}} \cdot \frac{T_{r e v}}{\tau_{i}} N_{\text {turn }}>-1 \\
\exp \left(\frac{b}{\varepsilon_{i}} \cdot \frac{T_{r e v}}{\tau_{i}} N_{\text {turn }}\right)-1, & \frac{b}{\varepsilon_{i}} \cdot \frac{T_{r e v}}{\tau_{i}} N_{\text {turn }} \leq-1
\end{array},\right.
$$

where $\theta_{i}$ - angles, $i=0 \div 2$ - index of degree of freedoms: $i=0$ - horizontal, $i=1-$ vertical, $i=2-$ longitudinal, $T_{\text {rev }}$ - revolution period, $\tau_{i}$ - heating growth rates, $N_{\text {turn }}$ - number of turns, $b=1$ for any case and $b=2$ for longitudinal direction in the case of a bunched beam. In the case of constant rates $\varepsilon_{i}=1$. In the case of linear rates $\varepsilon_{0}$ corresponds to horizontal emittance, $\varepsilon_{1}$ corresponds to vertical emittance, $\varepsilon_{2}$ corresponds to momentum spread in accordance with Eq.(1.3).

The diffusion heating applies random kicks to momentums of ions. In the case of diffusion heating with constant power the kick doesn't depend on the current values of emittances. In other case the diffusion heating kick depends on current emittances:

$$
\begin{aligned}
& \Delta \theta_{\text {hor }}=\sqrt{\frac{4}{\beta_{x}} \cdot \frac{T_{\text {rev }}}{\tau_{\text {hor }}} N_{\text {turn }} \cdot \varepsilon_{\text {hor }}^{a}} \cdot \xi \\
& \Delta \theta_{\text {ver }}=\sqrt{\frac{4}{\beta_{y}} \cdot \frac{T_{\text {rev }}}{\tau_{\text {ver }}} N_{\text {turn }} \cdot \varepsilon_{\text {ver }}^{a}} \cdot \xi \\
& \Delta \theta_{\text {lon }}=\sqrt{2 b \cdot \frac{T_{\text {rev }}}{\tau_{\text {lon }}} N_{\text {turn }} \cdot \varepsilon_{\text {lon }}^{a} \cdot \xi}
\end{aligned}
$$

where $\beta_{x}, \beta_{y}$ are horizontal and vertical beta functions, $a=1$ for diffusion heating with constant power and $a=-1$ for diffusion heating which depends on current emittances, $\xi$ is the random value with Gaussian distribution of unit dispersion.

\section{References}

[1] I.N. Meshkov, A.O. Sidorin, A.V. Smirnov, E.M. Syresin, G.V. Trubnikov, P.R. Zenkevich, "Simulation of Electron Cooling Process in Storage Rings Using BETACOOL Program" , Proceedings of Beam Cooling and Related Topics, Bad Honnef, Germany, 2001.

[2] V. Avilov, "Calculation of Electrostatic Energy of Planar Lattices", Solid State Communications, v.44, No.4 p.555-558 (1982).

[3] M. Martini. Intrabeam scattering in the ACOOL-AA machines. CERN PS/84-9 AA, Geneva (1984).

[4] V.V. Parkhomchuk, New insights in the theory of electron cooling, NIM A 441 (2000) 9-17, p.9 


\section{Part II. Electron cooling}

\section{Introduction}

To solve the problems related with the cooling process a hierarchy of objects was developed in the framework of the BETACOOL program. Structure of the electron cooling [1] effect permits to extract procedures at different levels and to include them into calculation of the cooling process in other programs. The cooling simulation is based on a friction force calculation in the particle rest frame. The next layer of the simulation is related to cooler representation as a map, transforming particle coordinates from the entrance to the exit of the cooling section. The map of the cooler can be used directly in the framework of the Molecular Dynamics algorithm, or in other tracking procedures. On the basis of the map one can calculate kick of the ion momentum after crossing the cooling section which is necessary for simulation of the ion distribution evolution within the Model Beam algorithm. The map of the cooler is also used for the calculation of the cooling rate which is necessary for RMS dynamics simulation.

In this chapter we describe structure of the electron cooler representation. Briefly we discuss the theory of the friction force calculation based on the binary collision model and describe analytical models for the friction force calculation used in BETACOOL. Calculation of the cooler map is based on a model of electron beam that provides transformation of the ion velocity to the frame related with the electron beam and takes into account real geometry of the cooler. There are several models of electron beam which are presently available for simulations. Algorithm for calculation of the ion momentum kick after crossing of the cooler is described in Section 5. The cooling rate calculation can be performed using two models of the ion beam - the cooling rates for "rms particle", or cooling rates for the ion beam with Gaussian distribution in all degrees of freedom (see Section 6).

\section{Structure of the algorithm}

The uniform way of the friction force calculation is an application of the corresponding formulae given in particle reference frame (PRF), which moves with average particle velocity $V_{0}$. For analytic expressions of the friction force the transformation of the ion velocity into PRF and the force components back to the laboratory reference frame (LRF) can be provided also analytically, so that one can use in the formulae written in LRF as well. However, direct numerical calculation of the friction force is usually provided in PRF. To have a possibility to use the same algorithm for analytic expressions and for results of numerical simulations, the transformation between the reference frames was realized as a part of the cooling calculation algorithm.

In this case, the calculation procedure requires transformation of certain parameters from LRF to PRF and, after the calculation of the friction force components in PRF, their transformation back to LRF. The ion velocity components $V_{x, z}$ in PRF are equal to

$$
V_{x, z}=\gamma \beta c \theta_{x, z}, \quad V_{s}=\beta c \theta_{s}
$$

To transform the friction force components from PRF to LRF the following expressions are used

$$
F_{x, z} \equiv\left(F_{x, z}\right)_{L R F}=\frac{1}{\gamma}\left(F_{x, z}\right)_{P R F}, F_{s} \equiv\left(F_{s}\right)_{L R F}=\left(F_{s}\right)_{P R F}
$$


Here $x, z$ are the horizontal and vertical co-ordinates, $\theta_{x, z}=\frac{P_{x, z}}{P}, \theta_{s}=\frac{\Delta P}{P}, P=\beta \gamma M c$ is the longitudinal component of the reference particle momentum, $P_{x, z}$ are the transverse components of the ion momentum, $\Delta P$ is the longitudinal momentum deviation, $s-s_{0}$ is the ion longitudinal distance from the bunch center (the reference particle), $\beta=V_{0} / c, \gamma=\sqrt{1-\beta^{2}}, c$ is the speed of light, $V_{0}$ - velocity of the reference particle.

In PRF the friction force is a function of two components of the ion velocity: across and along the magnetic field line (or electron beam axis in the non-magnetized case) and the force has also only two components - transverse and longitudinal. The transverse component of the ion velocity is calculated as:

$$
v_{i, \perp}=\sqrt{V_{x}^{2}+V_{z}^{2}}
$$

The friction force for $x$ and $z$ components is calculated from the transverse component in accordance with:

$$
F_{x}=\frac{V_{x}}{v_{i, \perp}} F_{\perp}, F_{z}=\frac{V_{z}}{v_{i, \perp}} F_{\perp} .
$$

Such a model presumes that gradient of the electron beam density is negligible inside a region of effective interaction between ion and electrons.

In the present version of the program the friction force components in PRF can be calculated using one of the analytic formulae from the friction force library:

- Budker's,

- non magnetized,

- by Derbenev - Skrinsky - Meshkov,

- by Parkhomchuk,

- by Toepffer,

described in the next chapter. The friction force can be also represented by a numerical Table and read into the code from the file containing results of numerical calculations. Such force representation is called "Tabulated". In addition, the method "Electron array" allows one to calculate component of the force for an arbitrary electron distribution. The library is realized as a set of independent procedures. Each of them obtains at the entrance two components of the ion velocity and returns two components of the friction force. Each procedure uses for calculations the same list of input parameters. This list includes the following parameters of the ion and electron beam:

- the ion atomic and charge numbers,

- the magnetic field value,

- local electron transverse and longitudinal velocity spreads,

- local effective electron velocity spread,

- local electron density,

- the ion time of flight the cooling section,

- electron beam radius.

Each procedure of the friction force calculation does not require a total list of these parameters, but, for universal usage of the friction force, all the parameters should be determined before the friction force calculation. Transformation of the ion velocity from LRF to PRF, transformation of the friction force components from PRF to LRF, and calculation of the list of parameters for friction force calculation are provided by a model of the electron beam. 
A variety of models of the electron beam is available:

- cylinder of round cross-section with uniform density distribution,

- cylinder of elliptical cross-section and Gaussian density distribution of electrons in the transverse planes,

- ellipsoidal bunch with Gaussian density distribution in all co-ordinates.

- hollow transverse distribution of electron beam

- array of particles read from external file

First model of the electron beam corresponds to traditional electron cooling system and this model takes into account space charge effects in the electron beam. Second and third models are oriented to simulation of the electron cooling in $\mathrm{GeV}$ ion energy range. The elliptical cylinder can be used for modeling of coasting electron beam circulating in small ring. The Gaussian bunch corresponds to RF accelerated electron beam. Each model of the electron beam calculates the local parameters of the electron beam in the frame referenced to the electron beam orbit.

To take into account displacement and misalignment of the electron beam and influence of the magnetic field line curvature one needs to provide transformation of the ion co-ordinates and velocity components from the frame referenced to the ion beam equilibrium orbit to the frame referenced to the electron beam orbit and back. In the case of bunched ion and electron beams one needs to transform the ion longitudinal co-ordinate measured relatively to the center of the ion bunch to the distance from the center of the electron bunch. The model of the electron beam provides all these transformations also.

All the models of the electron beam are used in the same way: the model obtains at the entrance the ion coordinates in the form of vector:

$$
\vec{X}=\left\{x, \theta_{x}, z, \theta_{z}, s-s_{0}, \theta_{s}\right\},
$$

in the laboratory frame referenced to the ion beam equilibrium orbit and returns three components of the friction force in the same frame.

The electron cooler representation in the form of a map (which is necessary for tracking procedures) is based on the electron beam model. The right hand sides of the motion equation are calculated by an addition (to the force components) of the terms describing drift motion of the ion in the cooling section. Influence of the magnetic field and space charge fields of the electron beam as well as the ion motion distortion at the entrance of the cooling section can be taken into account also. The solution of the ion motion equation is provided by one of the numerical methods developed in the program. The ion motion equation can be solved in the thin lens approximation or by solution of the motion equation using Euler or 4th order Runge-Kutta method.

The map of the cooler obtains at the entrance the initial ion co-ordinates and returns the ion coordinates at the exit of the cooler and probability of the ion loss due to recombination with the electrons. The particle loss probability is calculated under assumption that the ion velocity is less than the one of electrons.

The map of the cooler is used as a basis for electron cooling representation as an effect acting on the ion distribution function. Electron cooling, as an effect, includes two standard procedures. One of them provides kick of the momentum components for all ions in the model beam and calculates the particle losses due to recombination in the cooling section. Other procedure calculates characteristic times of the ion beam rms emittance variation and the beam lifetime. The characteristic times can be calculated using two model of the ion beam: single particle cooling times and cooling times for 
Gaussian beam obtained using Monte-Carlo method. Electron cooling, as an effect, obtains at the entrance the ion beam in one of the form using in the program and returns the ion beam in the same form with parameters changed by action of the electron cooling.

The described structure of the electron cooling object permits to use uniformly all the models developed at the same layer of the hierarchy. Each model at some level can use all the models at the lower layers in arbitrary combination. Each layer of the hierarchy can be extracted from the BETACOOL and used as an independent object in another program.

\section{Friction force in the particle rest frame}

\subsection{Non-magnetized electron beam}

\subsubsection{Binary collision model}

The friction force acting on ion is determined by Coulomb collisions with electrons (Fig. 3.1). The electron with velocity $v_{e}$ in the PRF colliding with the ion which has velocity $V$ at impact parameter $\rho$ obtains the transverse momentum $\Delta p_{\perp}$ relatively to the vector $\vec{U}=\vec{V}-\vec{v}_{e}$ :

$$
p_{\perp} \equiv \Delta p_{\perp}=\frac{2 Z e^{2}}{\left|\vec{V}-\vec{v}_{e}\right| \rho}
$$

$Z e, e$ are the charges of the ion and electron. Due to conservation of the total particle momentum $p_{\mu}$ = const the appearance of the transverse momentum $p_{\perp}$ of electron leads to the following change of its longitudinal momentum:

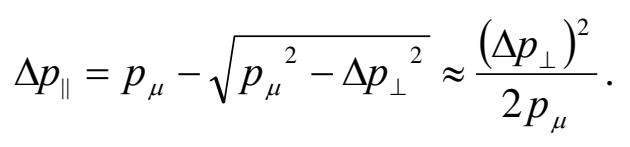

The electron energy changes by the value:

$$
\Delta E_{e}=\frac{\Delta p_{\perp}^{2}}{2 m}
$$

which is equal to the change of the ion energy $\Delta E_{i}$. Here $m$ is the electron mass.

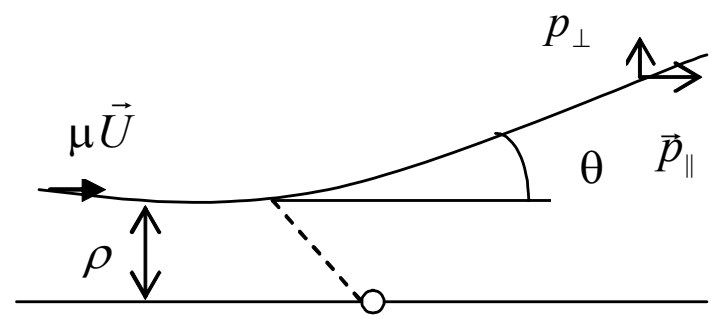

Fig. 3.1. Two-body problem 
Integration over impact parameter $\rho$ gives us the ion energy loss per unit of the length:

$$
\frac{d E_{i}}{d s}=2 \pi \int_{\rho_{\min }}^{\rho_{\max }} n_{e} \Delta E_{i} \rho d \rho
$$

Here $n_{e}$ is the electron density, $\rho_{\max }, \rho_{\min }-$ minimum and maximum impact parameters of the collision. The minimal impact parameter $\rho_{\min }$ approximately corresponds to electron scattering by the angle of $\pi / 2$. The maximum impact parameter corresponds to the distance between particles when the effective interaction is possible. The friction force in the extremely cold electron beam is equal to:

$$
F=-\frac{d E_{i}}{d s}=-\frac{4 \pi Z^{2} n_{e} e^{4}}{m V^{2}} \ln \frac{\rho_{\max }}{\rho_{\min }}
$$

\subsubsection{Numerical integration}

When the electrons are distributed over velocities in accordance with the function $f\left(v_{e}\right)$ the friction force can be evaluated by numerical integration of the following formula [2-5]:

$$
\vec{F}=-\frac{4 \pi n_{e} e^{4} Z^{2}}{m} \int \ln \left(\frac{\rho_{\max }}{\rho_{\min }}\right) \frac{\vec{V}-\vec{v}_{e}}{\left|\vec{V}-\vec{v}_{e}\right|^{3}} f\left(v_{e}\right) d^{3} v_{e}
$$

The Coulomb logarithm $\ln \frac{\rho_{\max }}{\rho_{\min }}$ is kept under the integral because the minimal impact parameter depends on electron velocity:

$$
\rho_{\min }=\frac{Z e^{2}}{m} \frac{1}{\left|\vec{V}-\vec{v}_{e}\right|^{2}} .
$$

In the case of uniform velocity distribution of electrons, the electron beam can be described by the temperature $T_{e}$ and r.m.s. electron velocity spread $\Delta_{e}$ over each of the three dimensions, that are connected with each other in accordance with

$$
T_{e}=m \Delta_{e}^{2}
$$

For a given value of the ion velocity the maximum impact parameter is constant and it is determined by dynamic shielding radius or the ion time of flight through the electron cloud. Radius of the dynamic shielding sphere coincides with Debay radius:

$$
\rho_{D}=\frac{\Delta_{e}}{\omega_{p}}
$$

when the ion velocity is less than the electron r.m.s velocity spread $\Delta_{e}$. The plasma frequency $\omega_{p}$ is equal to 


$$
\omega_{p}=\sqrt{\frac{4 \pi n_{e} e^{2}}{m}} .
$$

When the ion velocity is sufficiently larger than the electron velocity spread it determines the shielding radius

$$
\rho_{s h}=\frac{V}{\omega_{p}} .
$$

Both of the formulas (3.9) and (3.11) can be combined together to have a smooth dependence of the shielding radius on the ion velocity:

$$
\rho_{s h}=\frac{\sqrt{V^{2}+\Delta_{e}^{2}}}{\omega_{p}}
$$

In the case, when the shielding sphere does not contain sufficient number of electrons to compensate the ion charge (such a situation may take place in the case of magnetized electron beam with low longitudinal velocity spread) it has to be increased in accordance with the electron beam density and the ion charge. In the program this radius is estimated from the expression

$$
n_{e} \rho^{3} \sim 3 Z
$$

As a result, the maximum impact parameter is calculated as a minimum from three values:

$$
\rho_{\max }=\min \left\{\max \left(\rho_{s h}, \quad \sqrt[3]{\frac{3 Z}{n_{e}}}\right), V \tau\right\} .
$$

The second term describes the distance, which the ion passes inside the electron beam. Here $\tau$ is the ion time of flight the cooling section in the PRF:

$$
\tau=\frac{l_{\text {cool }}}{\beta \gamma c} \text {. }
$$

In the case of axial symmetry the electron distribution function can be written in the following form:

$$
f\left(v_{e}\right)=\left(\frac{1}{2 \pi}\right)^{3 / 2} \frac{1}{\Delta_{\perp}^{2} \Delta_{\|}} \exp \left(-\frac{v_{\perp}^{2}}{2 \Delta_{\perp}^{2}}-\frac{v_{\|}^{2}}{2 \Delta_{\|}^{2}}\right)
$$

where $\Delta_{\perp}$ and $\Delta_{\|}$are the electron rms velocity spreads in the transverse and longitudinal direction correspondingly.

Asymmetry of the electron distribution function can appear, for instance, due to electrostatic acceleration of the electron beam. In this case the temperatures of transverse and longitudinal degrees of freedom are different (see details in [4]): 


$$
T_{\perp} \approx T_{\text {cathode }}+T_{\text {optics }}, \quad T_{\|}=\frac{T_{\text {cathode }, \text { eff }}^{2}}{\beta^{2} \gamma^{2} m c^{2}}+e^{2} n_{e}^{1 / 3},
$$

where $T_{\text {cathode }}$ is the cathode temperature, $T_{\text {optics }}$ describes an additional transverse velocity spread due to distortions during electron beam transportation to the cooling section (this temperature includes also the incoherent drift motion in the crossed fields (see chapter 4.2.)). The effective cathode temperature used for longitudinal temperature calculation includes a term determined by amplitude of the accelerating voltage ripple.

In the general case, for example for RF electron beam acceleration, the temperatures of the transverse and longitudinal degrees of freedom can be calculated from electron beam parameters as follows:

$$
\begin{array}{r}
T_{\perp}=m c^{2} \beta^{2} \gamma^{2} \theta^{2}, \\
T_{\|}=m c^{2} \beta^{2}\left(\frac{\Delta p}{p}\right)^{2},
\end{array}
$$

where $\theta$ is r.m.s. angular spread and $\Delta p / p$ - r.m.s. momentum spread of electrons in the cooling section. The angular spread can be a function of radial co-ordinates due to the drift motion of electrons or oscillations of the beam envelope. The relation between temperatures and corresponding rms velocity spread in particle rest frame (PRF) is determined similarly to formula (3.8):

$$
\begin{aligned}
& T_{\|}=m \Delta_{\|}{ }^{2}, \\
& T_{\perp}=m \Delta_{\perp}{ }^{2} .
\end{aligned}
$$

The shielding cloud in the case of non uniform distribution has an ellipsoidal shape which can be approximated by the sphere of radius calculated using effective electron velocity spread:

$$
\Delta_{e}^{2}=\Delta_{\perp}^{2}+\Delta_{\|}^{2}
$$

The components of the friction force (3.1) can be calculated in cylindrical co-ordinate system as follows:

$$
\begin{aligned}
& F_{\perp}=-\sqrt{\frac{2}{\pi}} \frac{Z^{2} e^{4} n_{e}}{m \Delta_{\perp}^{2} \Delta_{\|}} \int_{0}^{\infty} \int_{-\infty}^{\infty} \int_{0}^{2 \pi} \ln \left(\frac{\rho_{\max }}{\rho_{\min }}\right) \frac{\left(V_{\perp}-v_{\perp} \cos \varphi\right) \exp \left(-\frac{v_{\perp}^{2}}{2 \Delta_{\perp}^{2}}-\frac{v_{\|}^{2}}{2 \Delta_{\|}^{2}}\right)}{\left(\left(V_{\|}-v_{\|}\right)^{2}+\left(V_{\perp}-v_{\perp} \cos \varphi\right)^{2}+v_{\perp}^{2} \sin ^{2} \varphi\right)^{3 / 2}} v_{\perp} d \varphi d v_{\|} d v_{\perp}, \\
& F_{\|}=-\sqrt{\frac{2}{\pi}} \frac{Z^{2} e^{4} n_{e}}{m \Delta_{\perp}^{2} \Delta_{\|}} \int_{0}^{\infty} \int_{-\infty}^{\infty} \int_{0}^{2 \pi} \ln \left(\frac{\rho_{\max }}{\rho_{\min }}\right) \frac{\left.v_{\|}^{2}\right) \exp \left(-\frac{v_{\perp}^{2}}{2 \Delta_{\perp}^{2}}-\frac{v_{\perp}}{2 \Delta_{\|}^{2}}\right)}{\left(\left(V_{\|}-v_{\|}\right)^{2}+\left(V_{\perp}-v_{\perp} \cos \varphi\right)^{2}+v_{\perp}^{2} \sin ^{2} \varphi\right)^{3 / 2}} v_{\perp} d \varphi d v_{\|} d v_{\perp} .
\end{aligned}
$$

In numerical calculations, within an accuracy of about $2 \%$ the upper limit of the integrals over velocity components can be replaced from infinity to three corresponding rms values and integration over $\varphi$ can be performed from 0 to $\pi$ due to symmetry of the formulae. In this case the friction force components can be calculated as: 


$$
\begin{aligned}
& F_{\perp}=-\frac{4 \pi Z^{2} e^{4} n_{e}}{m \cdot I n t} \int_{0}^{3 \Delta_{\perp}} \int_{-3 \Delta_{\|}}^{3 \Delta_{\|}} \int_{0}^{\pi} \ln \left(\frac{\rho_{\max }}{\rho_{\min }}\right) \frac{\left(V_{\perp}-v_{\perp} \cos \varphi\right) \exp \left(-\frac{v_{\perp}^{2}}{2 \Delta_{\perp}^{2}}-\frac{v_{\|}^{2}}{2 \Delta_{\|}^{2}}\right)}{\left(\left(V_{\|}-v_{\|}\right)^{2}+\left(V_{\perp}-v_{\perp} \cos \varphi\right)^{2}+v_{\perp}^{2} \sin ^{2} \varphi\right)^{3 / 2}} v_{\perp} d \varphi d v_{\|} d v_{\perp} \\
& F_{\|}=-\frac{4 \pi Z^{2} e^{4} n_{e}}{m \cdot \operatorname{Int}} \int_{0}^{3 \Delta_{\perp}} \int_{-3 \Delta_{\|}}^{3 \Delta_{\|}} \int_{0}^{\pi} \ln \left(\frac{\rho_{\max }}{\rho_{\min }}\right) \frac{\left(V_{\|}-v_{\|}\right) \exp \left(-\frac{v_{\perp}^{2}}{2 \Delta_{\perp}^{2}}-\frac{v_{\|}^{2}}{2 \Delta_{\|}^{2}}\right)}{\left(\left(V_{\|}-v_{\|}\right)^{2}+\left(V_{\perp}-v_{\perp} \cos \varphi\right)^{2}+v_{\perp}^{2} \sin ^{2} \varphi\right)^{3 / 2}} v_{\perp} d \varphi d v_{\|} d v_{\perp}
\end{aligned}
$$

where the normalization factor is calculated in accordance with:

$$
I n t=\int_{0}^{3 \Delta_{\perp}} \int_{-3 \Delta_{\|}}^{3 \Delta_{\|}} \int_{0}^{\pi} \exp \left(-\frac{v_{\perp}^{2}}{2 \Delta_{\perp}^{2}}-\frac{v_{\|}^{2}}{2 \Delta_{\|}^{2}}\right) v_{\perp} d \varphi d v_{\|} d v_{\perp} .
$$

The minimal impact parameter is the following function of the electron velocity components:

$$
\rho_{\min }=\frac{Z e^{2}}{m_{e}} \frac{1}{\left(V_{\|}-v_{\|}\right)^{2}+\left(V_{\perp}-v_{\perp} \cos \varphi\right)^{2}+v_{\perp}^{2} \sin ^{2} \varphi} .
$$

At the ion velocity $V>>\Delta_{\|}, \Delta_{\perp}$ the minimal impact parameter becomes to be constant:

$$
\rho_{\min }=\frac{Z e^{2}}{m_{e}} \frac{1}{V_{\perp}^{2}+V_{\|}^{2}},
$$

and Coulomb logarithm can be removed from the integral. At extremely small ion velocity the calculation of the minimal impact parameter in accordance with the formula (3.25) leads to zero friction force value, when $\rho_{\min }>\rho_{\max }$. One can avoid this problem introducing mean minimal impact parameter in accordance with

$$
\rho_{\min }=\frac{Z e^{2}}{m_{e}} \frac{1}{V_{\perp}^{2}+V_{\|}^{2}+\Delta_{\perp}^{2}+\Delta_{\|}^{2}} .
$$

When the Coulomb logarithm $L_{C}$ is constant the two of three integrals in (3.21) can be calculated analytically, and the friction force components can be written in accordance with Binney's formulae [3]:

$$
\begin{aligned}
& F_{\perp}=2 \sqrt{2 \pi} \frac{n_{e} e^{4} Z^{2} L_{C}}{m} \frac{V_{\perp}}{\Delta_{\perp}^{3}} I n t_{\perp} \\
& F_{\|}=2 \sqrt{2 \pi} \frac{n_{e} e^{4} Z^{2} L_{C}}{m} \frac{V_{\|}}{\Delta_{\perp}^{3}} I n t_{\|},
\end{aligned}
$$

where $I n t_{\perp}$ and $I n t_{\|}$are the following integrals: 


$$
\begin{aligned}
& I n t_{\perp}=\int_{0}^{\infty} \frac{\exp \left(-\frac{V_{\perp}^{2}}{2 \Delta_{\perp}^{2}} \frac{1}{1+q}-\frac{V_{\|}^{2}}{2 \Delta_{\perp}^{2}} \frac{1}{\left(\Delta_{\|} / \Delta_{\perp}\right)^{2}+q}\right)}{(1+q)^{2}\left(\left(\Delta_{\|} / \Delta_{\perp}\right)^{2}+q\right)^{1 / 2}} d q, \\
& \operatorname{Int}_{\|}=\int_{0}^{\infty} \frac{\exp \left(-\frac{V_{\perp}^{2}}{2 \Delta_{\perp}^{2}} \frac{1}{1+q}-\frac{V_{\|}^{2}}{2 \Delta_{\perp}^{2}} \frac{1}{\left(\Delta_{\|} / \Delta_{\perp}\right)^{2}+q}\right)}{(1+q)\left(\left(\Delta_{\|} / \Delta_{\perp}\right)^{2}+q\right)^{3 / 2}} d q \text {. }
\end{aligned}
$$

In the case of uniform Maxwellian distribution (when $\Delta_{\|}=\Delta_{\perp}=\Delta_{e}$ ) the integrals (3.28) coincide with each other and can be evaluated analytically, which was first done by Chandrasekhar [2]. This formula is implemented in BETACOOL under notation of Budker's formula for electron cooling time. The friction force in this case is given by:

$$
\begin{gathered}
\vec{F}=-\frac{\vec{V}}{V^{3}} \frac{4 \pi n_{e} e^{4} Z^{2} L_{C}}{m} \varphi\left(\frac{V}{\Delta_{e}}\right), \\
\text { where } \varphi(x)=\sqrt{\frac{2}{\pi}} \int_{0}^{x} e^{-y^{2} / 2} d y-\sqrt{\frac{2}{\pi}} x e^{-x^{2} / 2} .
\end{gathered}
$$

The formulae (3.22) give the same result when the logarithm is taken out of the integrals.

\subsubsection{Asymptotic representations for flattened velocity distribution}

For fast simulation of the cooling process one can use different asymptotic formulae. For example, the asymptotic formulas can be derived based on the Coulomb analogy (see Refs. [4]-[5]).

In the case, when transverse velocity spread of electrons is substantially larger than longitudinal one the friction force can be approximated in three ranges of the ion velocity. In accordance with Meshkov's asymptotes [4] the force components are calculated with the following formulae.

I. High velocity $V \geq \Delta_{\perp}$, here longitudinal and transverse components of the friction force are equal:

$$
\vec{F}=-\frac{4 \pi Z^{2} e^{4} n_{e} L_{C}}{m} \frac{\vec{V}}{V^{3}}
$$

and in this range the friction force shape coincides with formula (3.29).

II. Low velocity $\Delta_{\|} \leq V<\Delta_{\perp}$. Here, the transverse component of the friction force is given by the following expression:

$$
F_{\perp}=-\frac{4 \pi Z^{2} e^{4} n_{e} L_{C}}{m} \cdot \frac{V_{\perp}}{\Delta_{\perp}^{3}}
$$

and longitudinal one:

$$
F_{\|}=-\frac{4 \pi Z^{2} e^{4} n_{e} L_{C}}{m} \frac{V_{\|}}{\left|V_{\|}\right| \Delta_{\perp}^{2}} .
$$


III. Superlow velocity $V<\Delta_{\|}$. Here the transverse component of the friction force is equal to zero, the longitudinal component is given by:

$$
F_{\|}=-\frac{4 \pi Z^{2} e^{4} n_{e} L_{C}}{m} \frac{V_{\|}}{\Delta_{\|} \Delta_{\perp}^{2}} .
$$

The minimal impact parameter in the Coulomb logarithm is equal to:

$$
\rho_{\text {min }}=\frac{Z e^{2}}{m} \frac{1}{V^{2}+\Delta_{e}^{2}},
$$

where $\Delta_{e}$ is given by the formula (3.20).

The asymptotes by Ya. Derbenev for the longitudinal component of the force have the following form:

$$
\begin{gathered}
F_{\|}=-\frac{4 \pi Z^{2} e^{4} n_{e} L\left(V_{\|}\right)}{m} \frac{V_{\|}}{\Delta_{\|} \Delta_{\perp}^{2}} \sqrt{\frac{2}{\pi}}, \quad \text { if } V<\Delta_{\|} . \\
F_{\|}=-\frac{4 \pi Z^{2} e^{4} n_{e}}{m}\left[L\left(V_{\|}\right) \frac{V_{\|}}{\left|V_{\|}\right| \Delta_{\perp}^{2}}-\sqrt{\frac{\pi}{2}} L\left(\Delta_{\perp}\right) \frac{V_{\|}}{\Delta_{\perp}^{3}}\right], \quad \text { if } \Delta_{\perp}>V>>\Delta_{\|} .
\end{gathered}
$$

Here the Coulomb logarithms are calculated in accordance with the following formulae:

$$
\begin{aligned}
& L\left(V_{\|}\right)=\ln \left(\sqrt{\frac{V_{\|}^{2} m}{4 \pi n_{e} e^{2}}} / \rho_{\min }\right), \\
& L\left(\Delta_{\perp}\right)=\ln \left(\sqrt{\frac{\Delta_{\perp}^{2} m}{4 \pi n_{e} e^{2}}} / \rho_{\min }\right) .
\end{aligned}
$$

In order to provide uniform usage of the formulae in the program the friction force calculation is realized in three ranges of the ion velocity similarly to Meshkov's asymptotes.

I. High velocity $V \geq \Delta_{\perp}$, here longitudinal and transverse components of the friction force are equal:

$$
\begin{gathered}
F_{\perp}=-\frac{4 \pi Z^{2} e^{4} n_{e} L_{C}}{m} \frac{V_{\perp}}{V^{3}}, \\
F_{\|}=-\frac{4 \pi Z^{2} e^{4} n_{e}}{m}\left(L_{C} \frac{V_{\|}}{V^{3}}-\sqrt{\frac{2}{\pi}} L\left(\Delta_{\perp}\right) \frac{1}{V_{\|}^{2}}\right) .
\end{gathered}
$$

II. Low velocity $\Delta_{\|} \leq V<\Delta_{\perp}$. Here the transverse component of the friction force is given by the following expression: 


$$
F_{\perp}=-\frac{4 \pi Z^{2} e^{4} n_{e} L_{C}}{m} \cdot \frac{V_{\perp}}{\Delta_{\perp}^{3}},
$$

and longitudinal one:

$$
F_{\|}=-\frac{4 \pi Z^{2} e^{4} n_{e}}{m}\left(L_{C} \frac{V_{\|}}{\sqrt{V_{\|}^{2}+\Delta_{\|}^{2}} \Delta_{\perp}^{2}}-\sqrt{\frac{2}{\pi}} L\left(\Delta_{\perp}\right) \frac{V_{\|}}{\Delta_{\perp}^{3}}\right) .
$$

III. Superlow velocity $V<\Delta_{\|}$. Here the transverse component of the friction force is equal to zero, the longitudinal component is given by:

$$
F_{\|}=-\frac{4 \pi Z^{2} e^{4} n_{e}}{m} L_{C} \frac{V_{\|}}{\sqrt{V_{\|}^{2}+\Delta_{\|}^{2}} \Delta_{\perp}^{2}} .
$$

These formulae in the case of $V_{\perp}=0$ give the correct result for the longitudinal component of the friction force (3.45), (3.46) and have a correct asymptotes at high ion velocity. The transverse component of the force is calculated in accordance with Meshkov's representation.

\subsection{Magnetized electron beam.}

In the magnetized electron beam, when the maximum impact parameter (3.14) is larger than radius of electron Larmor rotation so called "magnetized collisions" between ion and electron take place. In this case the electron is attracted by the ion, which pulls it along the magnetic field line [5]. In different ranges of the ion velocity and impact parameter three type of collisions are possible: fast, adiabatic and magnetized.

\subsubsection{Magnetized collisions}

Practical analytic expressions for the friction force in strong magnetic field were worked out by Ya. Derbenev and A. Skrinsky [5]. For ion collisions with electrons at the impact parameter higher than the mean radius of electron Larmor rotation

$$
\rho_{\perp}=\frac{c m \Delta_{\perp}}{e B}
$$

the friction force in the particle rest frame can be expressed as follows [5]:

$$
\vec{F}=\frac{2 \pi Z^{2} e^{4} n_{e}}{m} \frac{\partial}{\partial \vec{V}} \int\left[\frac{V_{\perp}^{2}}{U^{3}} L_{M}+\frac{2}{U}\right] f\left(v_{e}\right) d v_{e},
$$

where $U=\sqrt{V_{\perp}^{2}+\left(V_{\|}-v_{e}\right)^{2}}$ - the relative velocity of the ion and electron "Larmor circle". $f\left(v_{e}\right)$ is the electron distribution over longitudinal velocity, in the case of Maxwellian distribution with rms velocity spread of $\Delta_{\|}$it is expressed as

$$
f\left(v_{e}\right)=\frac{1}{\sqrt{2 \pi \Delta_{\|}}} \exp \left(-\frac{v_{e}^{2}}{2 \Delta_{\|}^{2}}\right)
$$


Maximum impact parameter in the Coulomb logarithm for magnetized collisions

$$
L_{M}=\ln \left(\frac{\rho_{\max }}{\rho_{\perp}}\right)
$$

is calculated as usual (3.14).

The formula (3.45) has asymptotes in the region of small and large ion velocities. When $\mathrm{V}>\Delta_{\|}$ the electron distribution can be approximated by delta-function $f\left(v_{e}\right)=\delta\left(v_{e}\right)$, and integration in (3.45) gives:

$$
\begin{aligned}
& F_{\|}=-V_{\|} \frac{2 \pi Z^{2} e^{4} n_{e}}{m V^{3}}\left(\frac{3 V_{\perp}^{2}}{V^{2}} L_{M}+2\right), \\
& F_{\perp}=-V_{\perp} \frac{2 \pi Z^{2} e^{4} n_{e} L_{M}}{m V^{3}} \frac{V_{\perp}^{2}-2 V_{\|}^{2}}{V^{2}} .
\end{aligned}
$$

When the ion velocity is sufficiently less then electron velocity spread $\mathrm{V}<<\Delta_{\|}$the friction force can be expressed as [5]:

$$
\begin{gathered}
F_{\|} \approx-2 \sqrt{2 \pi \frac{Z^{2} e^{4} L_{M}}{m \Delta_{\|}^{3}} V_{\|},} \\
F_{\perp} \approx-2 \sqrt{2 \pi} \frac{Z^{2} e^{4} L_{M}}{m \Delta_{\|}^{3}} \ln \left(\frac{\Delta_{\|}}{V_{\perp}}\right) V_{\perp} .
\end{gathered}
$$

In the general case the friction force components can be calculated numerically as the following 1dimensional integrals (see Eq. (3.45)):

$$
\begin{gathered}
F_{\|}\left(V_{\perp}, V_{\|}\right)=-\frac{2 \pi Z^{2} e^{4} n_{e}}{m} \int\left(L_{M} \frac{3 V_{\perp}^{2}\left(V_{\|}-v_{e}\right)}{\left(V_{\perp}^{2}+\left(V_{\|}-v_{e}\right)^{2}\right)^{5 / 2}}+2 \frac{V_{\|}-v_{e}}{\left(V_{\perp}^{2}+\left(V_{\|}-v_{e}\right)^{2}\right)^{3 / 2}}\right) f\left(v_{e}\right) d v_{e} . \\
F_{\perp}\left(V_{\perp}, V_{\|}\right)=-\frac{2 \pi Z^{2} e^{4} n_{e} L_{M}}{m} \int \frac{V_{\perp}\left(V_{\perp}^{2}-2\left(V_{\|}-v_{e}\right)^{2}\right)}{\left(V_{\perp}^{2}+\left(V_{\|}-v_{e}\right)^{2}\right)^{5 / 2}} f\left(v_{e}\right) d v_{e}
\end{gathered}
$$

Note, that Eq. (3.45) was derived using the approach of dielectric linear plasma response. The resulting non-logarithmic term in Eq. (3.45) and (3.52) is due to collective plasma waves. It should be taken into account only at large ion velocity $\mathrm{V} \gg \Delta_{\|}$. In the region of small ion velocity the longitudinal component of the friction force is

$$
F_{\|}\left(V_{\perp}, V_{\|}\right)=-\frac{2 \pi Z^{2} e^{4} n_{e} L_{M}}{m} \int \frac{3 V_{\perp}^{2}\left(V_{\|}-v_{e}\right)}{\left(V_{\perp}^{2}+\left(V_{\|}-v_{e}\right)^{2}\right)^{5 / 2}} f\left(v_{e}\right) d v_{e} .
$$

General problem in numerical integration of $(3.52,3.53,3.54)$ is singularity of the integrants at $\mathrm{V}_{\perp}, \mathrm{V}_{\|} \rightarrow 0$. In the integral (3.54) $\mathrm{V}_{\perp}$ can be moved out from the integration

$$
\int \frac{\left(V_{\|}-v_{e}\right)}{\left(V_{\perp}^{2}+\left(V_{\|}-v_{e}\right)^{2}\right)^{5 / 2}} \exp \left(-\frac{v_{e}^{2}}{2 \Delta_{\|}^{2}}\right) d v_{e}
$$

but one gets singularity at $\mathrm{V}_{\perp}=0$. However, the friction force at zero ion transverse velocity has a finite value given by the formula: 


$$
F_{\|}\left(0, V_{\|}\right)=-V_{\|} \frac{4 \pi Z^{2} e^{4} n_{e} L_{M}}{m \sqrt{2 \pi} \Delta_{\|}^{3}} \exp \left(-\frac{V_{\|}^{2}}{2 \Delta_{\|}^{2}}\right) .
$$

To avoid numerical difficulties the integrals $(3.52,3.54)$ can be replaced by their asymptotes $(3.50$, 3.51) at the ion velocity region $\mathrm{V} \ll \Delta_{\|}$and integral (3.54) can be replaced by its accurate value (3.56) at $\mathrm{V}_{\perp} \ll \Delta_{\|}$.

One can avoid numerical problems in integration of $(3.52,3.54)$ at small ion velocity and provide calculations without usage of analytic asymptotes using algorithm proposed by D. Pestrikov [6]. The integral (3.55) can be rewritten in the following form:

$$
\begin{aligned}
& F_{\|}\left(V_{\perp}, V_{\|}\right)=-\frac{2 \pi Z^{2} e^{4} n_{e} L_{M}}{m} \int \frac{V_{\perp}^{2}}{\left(V_{\perp}^{2}+\left(V_{\|}-v_{e}\right)^{2}\right)^{3 / 2}} \frac{d f\left(v_{e}\right)}{d v_{e}} d v_{e}, \\
& F_{\perp}\left(V_{\perp}, V_{\|}\right)=-\frac{2 \pi Z^{2} e^{4} n_{e} L_{M}}{m} \int \frac{V_{\perp}\left(V_{\|}-v_{e}\right)}{\left(V_{\perp}^{2}+\left(V_{\|}-v_{e}\right)^{2}\right)^{3 / 2}} \frac{d f\left(v_{e}\right)}{d v_{e}} d v_{e} .
\end{aligned}
$$

Using (3.56) and substitutions $x=\frac{v_{e}}{\Delta_{\|}}, y=\frac{V_{\|}}{\Delta_{\|}}, z=\frac{V_{\perp}}{\Delta_{\|}}$one can write these integrals in the following form:

$$
\begin{aligned}
& F_{\|}=-\frac{2 \pi Z^{2} e^{4} n_{e} L_{M}}{m \sqrt{2 \pi} \Delta_{\|}^{2}} \int_{-\infty}^{\infty} \frac{z^{2} x}{\left(z^{2}+(y-x)^{2}\right)^{3 / 2}} \exp \left(-\frac{x^{2}}{2}\right) d x, \\
& F_{\perp}=-\frac{2 \pi Z^{2} e^{4} n_{e} L_{M}}{m \sqrt{2 \pi} \Delta_{\|}^{2}} \int_{-\infty}^{\infty} \frac{z y x}{\left(z^{2}+(y-x)^{2}\right)^{3 / 2}} \exp \left(-\frac{x^{2}}{2}\right) d x,
\end{aligned}
$$

Changing the integration variable by $x=y+|z| \tan \alpha$ one can reduce these integrals to the form with nonsingular integrant:

$$
\begin{gathered}
F_{\|}=-\frac{2 \pi Z^{2} e^{4} n_{e} L_{M}}{m \sqrt{2 \pi} \Delta_{\|}^{2}} \int_{-\pi / 2}^{\pi / 2}(y \cos \alpha+z \sin \alpha) \exp \left(-\frac{(y+|z| \tan \alpha)^{2}}{2}\right) d \alpha, \\
F_{\perp}=-\operatorname{sgn}(z) \frac{2 \pi Z^{2} e^{4} n_{e} L_{M}}{m \sqrt{2 \pi \Delta_{\|}^{2}}} \int_{-\pi / 2}^{\pi / 2} \tan \alpha(y \cos \alpha+z \sin \alpha) \exp \left(-\frac{(y+|z| \tan \alpha)^{2}}{2}\right) d \alpha .
\end{gathered}
$$

Integral (3.61) at $z=0$ can be calculated analytically [6] and gives the formula (3.56).

\subsubsection{Friction force at small impact parameters}

When the impact parameter is less than the radius of the electron Larmor rotation $\rho<\rho_{\perp}$ the influence of the magnetic field can be neglected and the friction force can be calculated in accordance with:

$$
\vec{F}=-\frac{4 \pi n_{e} e^{4} Z^{2}}{m} \int\left(L_{F}+N_{c o l l} L_{A}\right) \frac{\vec{V}-\vec{v}_{e}}{\left|\vec{V}_{i}-\vec{v}_{e}\right|^{3}} f\left(v_{e}\right) d^{3} v_{e}
$$


The range of impact parameters from $\rho_{\min }$ to $\rho_{\perp}$ in (3.63) is divided by two regions: the region where

$$
\rho_{\min }<\rho<\rho_{F}
$$

corresponds to so called "fast collisions" and in the region of

$$
\rho_{F}<\rho<\rho_{\perp}
$$

the ion can collide with the same electron a few times during its movement through the cooling section. The last region corresponds to so called "adiabatic" or "cycling" collisions. The intermediate impact parameter $\rho_{F}$ can be estimated as the following:

$$
\rho_{F}=\frac{\sqrt{V^{2}+\Delta_{\|}^{2}}}{\omega_{B}} \equiv \rho_{\perp} \frac{\sqrt{V^{2}+\Delta_{\|}^{2}}}{\Delta_{\perp}},
$$

and corresponding Coulomb logarithms are:

$$
L_{A}=\ln \frac{\rho_{\perp}}{\rho_{F}}, L_{F}=\ln \frac{\rho_{F}}{\rho_{\min }} .
$$

The number of multiple adiabatic collisions of the ion with the same electron is [4]:

$$
N_{\text {coll }} \cong 1+\frac{\Delta_{\perp}}{\pi \sqrt{V^{2}+\Delta_{\|}^{2}}} .
$$

The minimum impact parameter is calculated as usual:

$$
\rho_{\min }=\frac{Z e^{2}}{m} \frac{1}{\left|\vec{V}-\vec{v}_{e}\right|^{2}} .
$$

\subsubsection{Asymptotic representation [4]}

Summarizing asymptotic presentations for all types of collisions one can write [4]:

$$
F_{\perp} \approx-\frac{2 \pi Z^{2} e^{4} n_{e}}{m} v_{\perp}\left\{\begin{array}{c}
\frac{1}{v^{3}}\left(2 L_{F}+\frac{V_{\perp}^{2}-2 V_{\|}^{2}}{V^{2}} L_{M}\right),\{I\} \\
\frac{2}{\Delta_{\perp}^{3}}\left(L_{F}+N_{c o l} L_{A}\right)+\frac{V_{\perp}^{2}-2 V_{\|}^{2}}{V^{2}} \frac{L_{M}}{V^{3}},\{I I\} \\
\frac{2}{\Delta_{\perp}^{3}}\left(L_{F}+N_{c o l} L_{A}\right)+\frac{L_{M}}{\Delta_{\|}^{3}},\{I I I\}
\end{array}\right.
$$




$$
F_{\|} \approx-\frac{2 \pi Z^{2} e^{4} n_{e}}{m} v_{\|}\left\{\begin{array}{c}
\frac{1}{v^{3}}\left(2 L_{F}+\frac{3 V_{\perp}^{2}}{V^{2}} L_{M}+2\right),\{I\} \\
\frac{2}{\Delta_{\perp}^{2} V_{\|}}\left(L_{F}+N_{c o l} L_{A}\right)+\left(\frac{3 V_{\perp}^{2}}{V^{2}} L_{M}+2\right) \frac{1}{V^{3}},\left\{I I_{a}\right\} \\
\frac{2}{\Delta_{\perp}^{2} \Delta_{\|}}\left(L_{F}+N_{c o l} L_{A}\right)+\frac{L_{M}}{\Delta_{\|}^{3}},\left\{I I_{b}, I I I\right\}
\end{array}\right.
$$

Ion velocity domains $I, I I=I I_{a}+I I_{b}$, and $I I I$ are shown in Fig. 3.3.

In these formulae the Coulomb logarithms are defined as follows:

$$
L_{M}=\ln \frac{R}{k \rho_{\perp}}, L_{A}=\ln \frac{k \rho_{\perp}}{\rho_{F}}, L_{F}=\ln \frac{\rho_{F}}{\rho_{\min }} .
$$

Note that if argument of the logarithm is less than 1, then the logarithm value has to be set to zero. It means that the corresponding type of collisions is absent at given parameters. The minimum impact parameter is given by the formula

$$
\rho_{\min }=\frac{Z e^{2}}{m_{e}} \frac{1}{V^{2}+\Delta_{\|}^{2}},
$$

and dynamic shielding radius in the formula (3.12) for the maximum impact parameter is determined by the electron longitudinal velocity spread:

$$
\rho_{s h}=\frac{\sqrt{V^{2}+\Delta_{\|}^{2}}}{\omega_{p}} \text {. }
$$

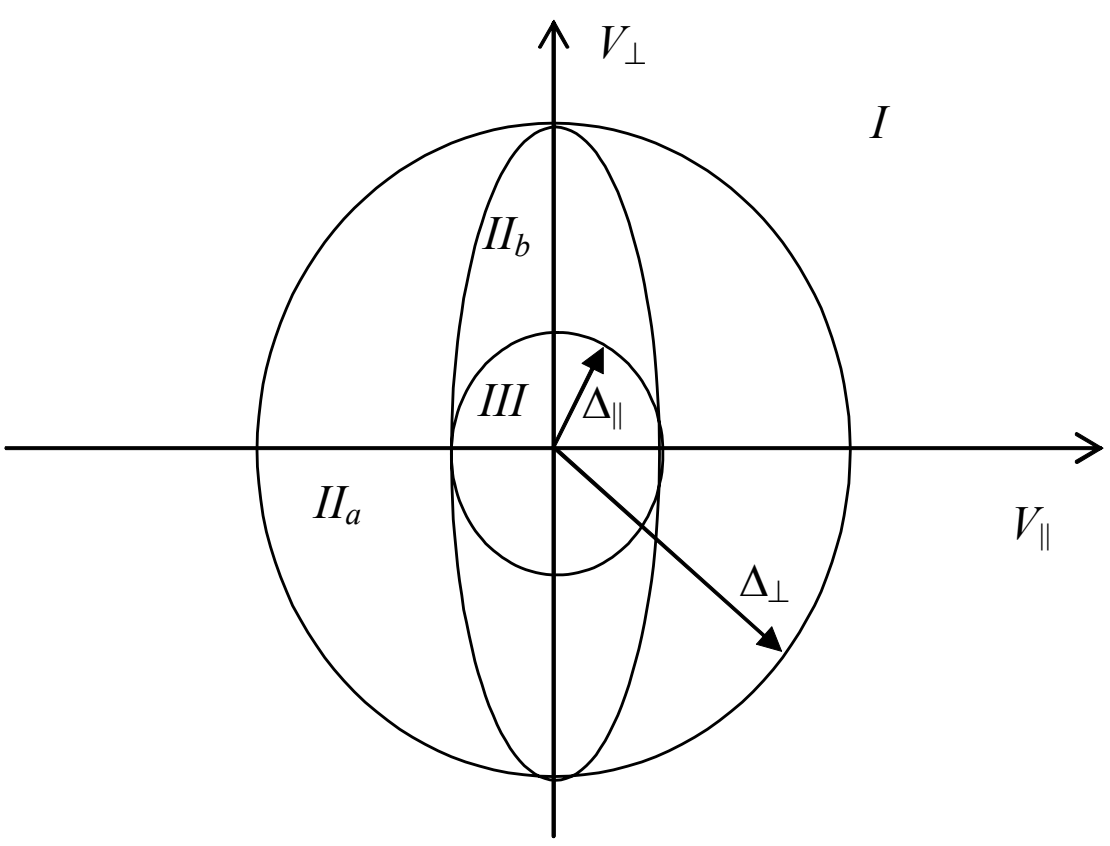

Fig.3.3. Domains in the velocity space for the friction force calculation. $V_{\perp}, V_{\|}$are the ion velocity components in PRF; $\Delta_{\perp}$ is the transverse electron velocity spread, $\Delta_{\|}$is the longitudinal one.

Coefficient $k$ in the formulae (3.70) was introduced to smooth the friction force shape. In the original paper it was chosen to be 2 , in the program now it is used as an additional input parameter. 


\subsubsection{Formula by Erlangen University}

The BETACOOL code also includes the friction force formula calculated by C. Toepffer (Erlangen Univ.) in the framework of binary collision model [13]. The unperturbed motion of electron is a helix with the Larmor radius:

$$
\rho_{\perp}=\frac{c m v_{\perp}}{e B}
$$

and the pitch determined by longitudinal velocity. The ion velocity variation is calculated iteratively and at impact parameters larger than the Larmor radius one can obtain solution in a closed form for two limiting cases:

$$
\delta=\frac{c m \sqrt{V_{\perp}^{2}+\left(V_{\|}-v_{\|}\right)^{2}}}{e B}>>\rho_{\perp}
$$

and $\delta<\rho_{\perp}$, where $\delta$ is the pitch of the helix as seen from the ion.

Correspondingly, the friction force includes three components related to different types of collision:

- fast collisions at impact parameters less than radius of electron rotation

- collisions with "tight" helices,

- collisions with "stretched" helices.

In the case of axial symmetry the electron distribution function is described by (3.16). For the fast collisions the formula is analogous to the non-magnetized collisions. The components of the friction force for fast collisions can be calculated in cylindrical coordinate system by Formulae (3.21). However, here both impact parameters - minimum and maximum - are now the functions of the electron velocity:

$$
\begin{gathered}
\rho_{\text {min }}=\frac{Z e^{2}}{m_{e}} \frac{1}{\left(V_{\|}-v_{\|}\right)^{2}+\left(V_{\perp}-v_{\perp} \cos \varphi\right)^{2}+v_{\perp}^{2} \sin ^{2} \varphi}, \\
\rho_{\text {max }}=\rho_{\perp}=\frac{c m v_{\perp}}{e B} .
\end{gathered}
$$

The friction force in collisions with tight helices [13]:

$$
\begin{aligned}
F_{\|, t}\left(V_{\perp}, V_{\|}\right) & =-\frac{4 \pi Z^{2} e^{4} n_{e} \quad 1}{m \quad \Delta_{\perp}^{2} \sqrt{2 \pi} \Delta_{\|}} \int \frac{V_{\perp}^{2}\left(V_{\|}-v_{\|}\right)}{\left(V_{\perp}^{2}+\left(V_{\|}-v_{\|}\right)^{2}\right)^{5 / 2}} \exp \left(-\frac{v_{\|}^{2}}{2 \Delta_{\|}^{2}}\right) \times \\
& \times \int_{0}^{\infty} \ln \left(\frac{\rho_{\max }}{\max \left(\rho_{\perp}, \delta\right)}\right) \exp \left(-\frac{v_{\perp}^{2}}{2 \Delta_{\perp}^{2}}\right) v_{\perp} d v_{\perp} d v_{\|}, \\
F_{\perp, t}\left(V_{\perp}, V_{\|}\right)= & -\frac{4 \pi Z^{2} e^{4} n_{e} 1}{m \Delta_{\perp}^{2} \sqrt{2 \pi} \Delta_{\|}} \int \frac{V_{\perp}\left(V_{\perp}^{2}-\left(V_{\|}-v_{\|}\right)^{2}\right)}{2\left(V_{\perp}^{2}+\left(V_{\|}-v_{\|}\right)^{2}\right)^{5 / 2}} \exp \left(-\frac{v_{\|}^{2}}{2 \Delta_{\|}^{2}}\right) \times \\
& \times \int_{0}^{\infty} \ln \left(\frac{\rho_{\max }}{\max \left(\rho_{\perp}, \delta\right)}\right) \exp \left(-\frac{v_{\perp}^{2}}{2 \Delta_{\perp}^{2}}\right) v_{\perp} d v_{\perp} d v_{\|},
\end{aligned}
$$


where

$$
\delta=\frac{c m \sqrt{V_{\perp}^{2}+\left(V_{\|}-v_{\|}\right)^{2}}}{e B} .
$$

For stretched helices [13]:

$$
\begin{aligned}
& F_{\|, s}\left(V_{\perp}, V_{\|}\right)=-\frac{4 \pi Z^{2} e^{4} n_{e}}{m} \frac{1}{\Delta_{\perp}^{2} \sqrt{2 \pi} \Delta_{\|}} \int \frac{V_{\|}-v_{\|}}{\left(V_{\perp}^{2}+\left(V_{\|}-v_{\|}\right)^{2}\right)^{3 / 2}} \exp \left(-\frac{v_{\|}^{2}}{2 \Delta_{\|}^{2}}\right) \\
& \int_{0}^{\infty} \ln \left(\frac{\min \left(\delta, \rho_{\max }\right)}{\min \left(\rho_{\perp}, \rho_{\max }\right)}\right) \exp \left(-\frac{v_{\perp}^{2}}{2 \Delta_{\perp}^{2}}\right) v_{\perp} d v_{\perp} d v_{\|} \text {, } \\
& F_{\perp, s}\left(V_{\perp}, V_{\|}\right)=-\frac{4 \pi Z^{2} e^{4} n_{e}}{m} \frac{V_{\perp}}{\Delta_{\perp}^{2} \sqrt{2 \pi \Delta_{\|}}} \int \frac{1}{\left(V_{\perp}^{2}+\left(V_{\|}-v_{\|}\right)^{2}\right)^{3 / 2}} \exp \left(-\frac{v_{\|}^{2}}{2 \Delta_{\|}^{2}}\right) \\
& \int_{0}^{\infty} \ln \left(\frac{\min \left(\delta, \rho_{\max }\right)}{\min \left(\rho_{\perp}, \rho_{\max }\right)}\right) \exp \left(-\frac{v_{\perp}^{2}}{2 \Delta_{\perp}^{2}}\right) v_{\perp} d v_{\perp} d v_{\|} \text {. }
\end{aligned}
$$

When $\mathrm{V}>>\Delta_{\|}$the electron distribution can be approximated by delta-function $f\left(v_{\|}\right)=\delta\left(v_{\|}\right)$. In this case integration over electron velocity components can be provided independently. The friction force components for tight helices can be expressed in the following form:

$$
\begin{gathered}
F_{\|}=-V_{\|} \frac{4 \pi Z^{2} e^{4} n_{e}}{m V^{3}} \frac{V_{\perp}^{2}}{V^{2}} L_{M}, \\
F_{\perp}=-V_{\perp} \frac{4 \pi Z^{2} e^{4} n_{e} L_{M}}{m V^{3}} \frac{V_{\perp}^{2}-V_{\|}^{2}}{V^{2}} .
\end{gathered}
$$

Here, the Coulomb logarithm is determined by the expression

$$
L_{M}=\frac{1}{\Delta_{\perp}^{2}} \int_{0}^{\infty} \ln \left(\frac{\rho_{\max }}{\max \left(\rho_{\perp}, \delta\right)}\right) \exp \left(-\frac{v_{\perp}^{2}}{2 \Delta_{\perp}^{2}}\right) v_{\perp} d v_{\perp} \approx \ln \left(\frac{\rho_{\text {max }}}{\left\langle\rho_{\perp}\right\rangle}\right),
$$

at $\delta=\frac{c m \sqrt{V_{\perp}^{2}+V_{\|}^{2}}}{e B}$. Within an accuracy of definition of the logarithm these formulae coincide with the one derived in the limit of an infinite magnetic field using binary collision approach by $\mathrm{V}$. Parkhomchuk [14]. Note, that apart from the non-logarithmic term, which comes from collective plasma response and thus is absent in the binary collision approach, Eq. (3.80)-(3.81) are similar to Eqs. (3.48)-(3.49) obtained via dielectric plasma approach [5]. The remaining difference is attributed to different cutoff parameters being used in two different approaches [16].

In the same approximation $V \gg \Delta_{\|}$the formulae for collisions with stretched helices can be rewritten in the form:

$$
\vec{F} \approx-\vec{V} \frac{4 \pi Z^{2} e^{4} n_{e}}{m} \frac{1}{\left(V^{2}+\Delta_{\|}^{2}\right)^{3 / 2}}\left(\ln \left(\frac{\rho_{\max }}{\left\langle\rho_{\perp}\right\rangle}\right)+\ln \left(\frac{\omega_{p}}{\omega_{B}}\right)\right),
$$


where $\omega_{p}, \omega_{B}$ are the plasma and cyclotron frequencies. This formula is valid at $\frac{V}{\Delta_{\perp}}>>1$ and its structure is similar to semi-empirical formula by Parkhomchuk [7].

\subsubsection{Semi-empirical formula by Parkhomchuk}

A semi-empirical formula for calculation of the friction force in magnetized electron beam can be written as [7]:

$$
\vec{F}=-\vec{V} \frac{4 Z^{2} e^{4} n_{e} L_{P}}{m} \frac{1}{\left(V^{2}+\Delta_{e, e f f}^{2}\right)^{3 / 2}},
$$

where $\Delta_{e, e f f}$ is the effective electron velocity spread with taking into account variations of the magnetic field line displacement ("oscillation") in the transverse direction. The Coulomb logarithm is given by the expression:

$$
L_{P}=\ln \left(\frac{\rho_{\max }+\rho_{\min }+\rho_{\perp}}{\rho_{\min }+\rho_{\perp}}\right)
$$

Here the minimum impact parameter is calculated in accordance with

$$
\rho_{\min }=\frac{Z e^{2}}{m} \frac{1}{V^{2}+\Delta_{e, e f f}^{2}} .
$$

Maximum impact parameter in accordance with original formula is calculated as:

$$
\rho_{\max }=\frac{v_{i}}{1 / \tau_{\text {flight }}+\omega_{p}},
$$

where $\tau_{\text {flight }}$ is the ion time of flight the cooling section (3.15), $\omega_{p}$ is the plasma frequency (3.10).

This formula presumes that

$$
\Delta_{e, e f f}<<\Delta_{\perp}
$$

Recently, the accuracy of available analytic representations of the magnetized friction force was explored in detail via direct numerical simulations. The results and conclusions about available formulas are summarized in Ref. [8].

\subsection{3-D non-magnetized force and arbitrary distribution}

\subsubsection{3-D force}

In addition to analytic formulae for the non-magnetized and magnetized friction force which can be used in BETACCOL (described in Sections 3.1-3.2), a numerical algorithm was recently 
implemented in BETACOOL which allows to calculated friction force for an arbitrary distribution of electrons, represented by an array of particles.

In the absence of longitudinal magnetic field in the cooling section the electron motion in transverse planes is uncoupled. Correspondingly, the electron bunch can have different velocity spreads in horizontal and vertical planes. In this case the friction force can not be presented as a sum of radial and longitudinal components, but it is a vector with all three different components. The components of 3D friction force can be calculated as an integral over electron velocity for a given distribution function. In the case of Gaussian electron bunch the distribution function in velocity can be approximated as

$$
f\left(v_{e}\right)=\left(\frac{1}{2 \pi}\right)^{3 / 2} \frac{1}{\Delta_{x} \Delta_{y} \Delta_{\|}} \exp \left(-\frac{v_{x}^{2}}{2 \Delta_{x}^{2}}-\frac{v_{y}^{2}}{2 \Delta_{y}^{2}}-\frac{v_{\|}^{2}}{2 \Delta_{\|}^{2}}\right),
$$

where $\Delta_{x, y, \|}$ are the electron velocity spreads in horizontal, vertical and longitudinal planes.

The friction force is calculated in accordance with the definition:

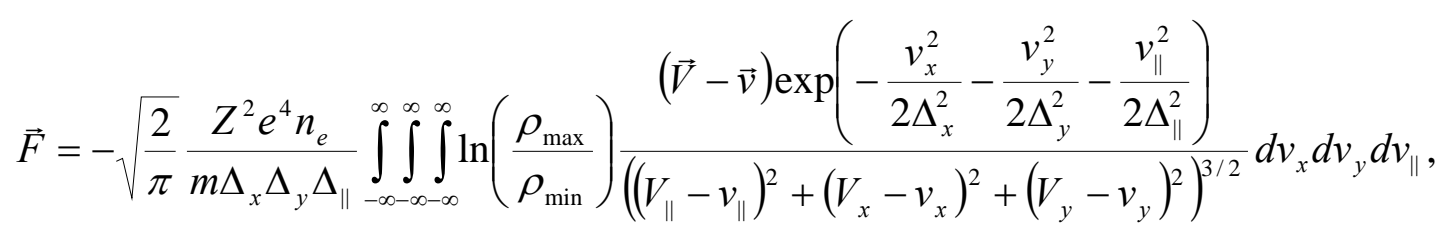

where minimum impact parameter is a function of the electron velocity $\vec{v}$ :

$$
\rho_{\min }=\frac{Z e^{2}}{m} \frac{1}{|\vec{V}-\vec{v}|^{2}} .
$$

The maximum impact parameter is calculated as usual:

$$
\rho_{\max }=\min \left\{\rho_{s h}, V \tau\right\}
$$

where the shielding radius is equal to $\rho_{s h}=\frac{\Delta_{e}}{\omega_{p}}$, when $V<\Delta_{e}$ and $\rho_{s h}=\frac{V}{\omega_{p}}$, when $V>\Delta_{e}$. Here $\Delta_{\mathrm{e}}$ is the total electron velocity spread:

$$
\Delta_{e}=\sqrt{\Delta_{x}^{2}+\Delta_{y}^{2}+\Delta_{\|}^{2}}
$$

and the plasma frequency is described by Formula (3.10):

$$
\omega_{p}=\sqrt{\frac{4 \pi n_{e} e^{2}}{m}} .
$$

In the case when undulator option is enabled the minimum impact parameter is calculated as:

$$
\rho_{\min }=\max \left(\rho_{\min }, \frac{e B \lambda^{2}}{4 \pi^{2} p c}\right) \text {, }
$$


where $B$ is the undulator field, $\lambda$ - its wavelength.

The 3D model of the friction force implemented in BETACOOL can be used correctly only in the case when electron beam is represented as an array of particles.

\subsubsection{Electron beam as an array of particles}

For the friction force calculation the program uses local parameters of the electron bunch calculated as functions of the ion co-ordinates. In the electron array the program finds $N_{l o c}$ electrons having minimum distance to the ion position. The value of $N_{l o c}$ is input in the edit window "Number of nearest particles". For obtained array of $N_{\text {loc }}$ electrons the program calculates mean and root mean square parameters for all the coordinates and velocity components.

The density and the velocity spreads evaluated for the local array can be used for the friction force calculation in accordance with the analytical formulae or asymptotic representation of the friction force. Usage of approximate formulae sufficiently speeds up the simulations but does not take into account asymmetry of the distribution function in the transverse plane. If the asymmetry is significant one can use formulae for 3D friction force. The corresponding electron rms velocity spreads are calculated as:

$$
\Delta_{x, y}=c \beta \gamma \sigma_{x^{\prime}, y^{\prime}},
$$

where $\sigma_{x^{\prime}, y^{\prime}}$ are the rms angular spreads of the local electrons.

Another possibility is to calculate the friction force using velocity components of the local electrons directly. For this purpose, the velocities of the local electrons are recalculated into the Particle Rest Frame. The distribution function of the local electrons in the velocity space is given as a series of $\delta$ - functions:

$$
f(v)=\frac{1}{N_{l o c}} \sum_{j=1}^{N_{\text {loc }}} \delta\left(\vec{v}-\vec{v}_{j}\right)
$$

Also, in the friction force expression, the integral over the distribution function is transformed into series. In this case the friction force components are calculated as follows:

$$
F_{\alpha}=\frac{4 \pi n_{e} Z^{2} e^{4}}{m} \frac{1}{N_{l o c}} \sum_{j=1}^{N_{\text {loc }}} \frac{\left(V_{\alpha}-v_{j, \alpha}\right) L_{C, j}}{\left.\left(V_{x}-v_{j, x}\right)^{2}+\left(V_{y}-v_{j, y}\right)^{2}+\left(V_{z}-v_{j, z}\right)^{2}\right)^{3}},
$$

where $V_{\alpha}$ are the components of ion velocity in the particle rest frame, $v_{j, \alpha}$ - the velocity components of $j$-th electron $(\alpha=\mathrm{x}, \mathrm{y}, \mathrm{z})$. The minimum impact parameter in the Coulomb logarithm $L_{C, j}$ is calculated via velocity of $j$-th electron:

$$
\rho_{\min , j}=\frac{Z e^{2}}{m} \frac{1}{\left|\vec{V}-\vec{v}_{j}\right|^{2}}
$$

There is a possibility to compare the cooling process dynamics for real (6-D) and Gaussian distribution of the electrons. BETACOOL generates an array with Gaussian distribution in all 
degrees of freedom, when the radio button "From file - Gaussian" is in the position "Gaussian" in the visual form. In this case, the rms values of electron co-ordinates and momentum components from the corresponding edit windows of the visual form are used as input parameters, as well as the number of particles in array, which determines dimension of the created array.

\subsection{Probability of the particle loss}

Probability in PRF of the electron capture by the ion during its passage in the cooling section is expressed by the following formula:

$$
\frac{d P_{l o s s}}{d t}=\frac{\alpha_{r} n_{e}}{\gamma^{2}}
$$

where $n_{e}$ is the local electron density in LRF. Under assumption that ion velocity in PRF is substantially less than the one of the electrons and for the flattened electron velocity distribution, the recombination coefficient $\alpha_{\mathrm{r}}$ is $[9,12]$ :

$$
\alpha_{r}=A \cdot Z^{2} \sqrt{\frac{1}{T_{\perp}}}\left[\ln \left(\frac{11.32 Z}{\sqrt{T_{\perp}}}\right)+0.14\left(\frac{T_{\perp}}{Z^{2}}\right)^{1 / 3}\right], A=3.02 \cdot 10^{-13} \frac{\mathrm{cm}^{3}}{\mathrm{~s}}
$$

In the case when the electron velocity spread depends on position inside the electron beam (for example, if space-charge effects in the electron beam are essential, or, when one uses results of the electron dynamics simulation performed using external program), the recombination coefficient as well as the local electron density is a function of the ion coordinates. Correspondingly, the particle loss probability after crossing the cooling section is calculated by numerical integration along the ion trajectory. This integration is performed together with solution of the ion motion equation in the cooling section, which is provided by a model of the electron beam. The algorithm is described in more details in the next sections.

\subsubsection{Simulation of ion-electron recombination in the presence of undulator field}

Electron cooling at RHIC [15] using non-magnetized electron beam sufficiently simplifies the cooler design. Generation and acceleration of the electron bunch without longitudinal magnetic field permits to reach low value of emittance in the cooling section. General problem of such a scheme is high recombination rate at low electron temperature. Suppression of the ion recombination with electrons in the cooling section using helical undulator field was proposed for RHIC in [11]. In the presence of the undulator field, trajectories of all electrons have the same coherent azimuth angle $\theta$, determined by the undulator period $\lambda$ and field value $B$ at the axis:

$$
\theta=\frac{e B \lambda}{2 \pi p c}
$$

where $p$ is the electron momentum. Since the recombination cross section is approximately inversely proportional to the electron energy in PRF, the ion beam life time can be sufficiently improved.

One can expect that at impact parameters significantly larger than electron rotation radius in helical undulator 


$$
r_{0}=\frac{\theta \lambda}{2 \pi}=\frac{e B \lambda^{2}}{4 \pi^{2} p c}
$$

kinematics of a binary collision will be similar to Rutherford scattering of a free electron. Thus the minimum impact parameter $\rho_{\min }$ in presence of the undulator field has to be replaced by $r_{0}$ value. At larger impact parameters the friction force can be calculated without taking into account coherent electron velocity.

The recombination coefficient is determined via recombination cross section $\sigma$ as

$$
\alpha_{r}=\int\left(V_{i}-v_{e}\right) \sigma\left(V_{i}-v_{e}\right) f\left(v_{e}\right) d^{3} v_{e},
$$

And has to be calculated taking into account the coherent transverse electron velocity. For the recombination rate calculation one can use the distribution function

$$
f(v) d^{3} v=\left(\frac{m}{2 \pi}\right)^{3 / 2} \frac{1}{T_{\perp} \sqrt{T_{I I}}} e^{-m\left(v_{\perp}+v_{\text {und }}\right)^{2} / 2 T_{\perp}-m v_{I I}^{2} / 2 T_{I I}} 2 \pi v_{\perp} d v_{\perp} d v_{I I}
$$

where $v_{\text {und }}$ is the electron azimuth velocity due to rotation in the undulator field:

$$
v_{\text {und }}=c \beta \gamma \theta
$$

The ion beam life time due to recombination in the cooling section is calculated via recombination coefficient $\alpha_{r}$ by the following formula:

$$
\frac{1}{N} \frac{d N}{d t}=-\frac{\alpha_{r} n_{e}}{\gamma^{2}} \frac{l_{c o o l}}{C}
$$

here $C$ is the ring circumference. Under assumption that ion velocity in PRF is substantially less than electron one the $\alpha_{r}$ is calculated in PRF by averaging of the recombination cross section over electron distribution function:

$$
\alpha_{r}=\langle v \sigma(v)\rangle
$$

where $v$ is the velocity of electrons.

The recombination cross section can be calculated with good accuracy using the following formula $[9,12]$ :

$$
\sigma=A\left(\frac{h v_{0}}{E}\right)\left(\ln \sqrt{\frac{h v_{0}}{E}}+0.1402+0.525\left(\frac{E}{h v_{0}}\right)^{1 / 3}\right)
$$


where $A=2^{4} 3^{-3 / 2} h e^{2} /\left(m_{e}^{3} c^{2}\right)=2.11 \times 10^{-22} \mathrm{~cm}^{2}, h v_{0}=13.6 \cdot Z^{2} \mathrm{eV}$ is the ion ground state binding energy. The electron kinetic energy $E=\frac{m_{e} v_{e}^{2}}{2}$. In presence of the undulator field it has to be calculated as:

$$
E=\frac{m}{2}\left(\left(v_{\perp}+v_{\text {und }}\right)^{2}+v_{\|}^{2}\right)
$$

The formula for flattened distribution can be rewritten in the form adopted for numerical integration:

$$
\alpha_{r}=\frac{1}{\operatorname{Int}} \int_{0}^{3 \Delta_{\perp}} \int_{-3 \Delta_{\|}}^{3 \Delta_{\|}} \sigma(E) \sqrt{\left(v_{\perp}+v_{u n d}\right)^{2}+v_{\|}^{2}} \exp \left(-\frac{\left(v_{\perp}+v_{u n d}\right)^{2}}{2 \Delta_{\perp}^{2}}-\frac{v_{\|}^{2}}{2 \Delta_{\|}^{2}}\right) v_{\perp} d v_{\|} d v_{\perp}
$$

The normalization factor is calculated as:

$$
\operatorname{Int}=\int_{0}^{3 \Delta_{\perp}} \int_{-3 \Delta_{\|}}^{3 \Delta_{\|}} \exp \left(-\frac{\left(v_{\perp}+v_{\text {und }}\right)^{2}}{2 \Delta_{\perp}^{2}}-\frac{v_{\|}^{2}}{2 \Delta_{\|}^{2}}\right) v_{\perp} d v_{\|} d v_{\perp}
$$

To avoid overflow in calculation of the exponents, at $v_{\text {und }}>6 \cdot \Delta_{\perp}$ the recombination coefficient is calculated directly from the coherent velocity:

$$
\alpha_{r}=v_{\text {und }} \sigma\left(v_{\text {und }}\right)
$$

that corresponds to electron distribution in the form of delta function.

In the absence of the undulator field the recombination coefficient $\alpha_{\mathrm{r}}$ for flattened electron velocity distribution is calculated using formula (3.102). 


\section{Map of the cooling section}

The map of the cooler provides transformation of the ion co-ordinates from the entrance to the end of the cooling section. This transformation is based on the solution of the ion motion equation in the cooling section. The ion motion inside a storage ring is described in the canonically conjugated variables:

$$
\vec{X}=\left\{x, \theta_{x}, z, \theta_{z}, s-s_{0}, \theta_{s}\right\},
$$

where $x, z$ are the horizontal and vertical co-ordinates, $\theta_{x, z}=\frac{P_{x, z}}{P}, \theta_{s}=\frac{\Delta P}{P}, P=\beta \gamma M c$ is the longitudinal component of the reference particle momentum, $P_{x, z}$ are the transverse components of the ion momentum, $\Delta P$ is the longitudinal momentum deviation, $s-s_{0}$ is the ion longitudinal distance from the bunch center (the reference particle), $\beta=V_{0} / c, \gamma=\sqrt{1-\beta^{2}}, c$ is the speed of light, $V_{0}$ - velocity of the reference particle.

Under assumption, that transverse components of the particle momentum are substantially less than longitudinal one, the ion motion equations can be presented in the following form:

$$
\left\{\begin{array}{c}
\frac{d x}{d s}=\theta_{x} \\
\frac{d \theta_{x}}{d s}=\frac{F_{x}}{M c^{2} \beta^{2} \gamma} \\
\frac{d z}{d s}=\theta_{z} \\
\frac{d \theta_{z}}{d s}=\frac{F_{z}}{M c^{2} \beta^{2} \gamma} \\
\frac{d\left(s-s_{0}\right)}{d s}=\frac{\theta_{s}}{\gamma^{2}} \\
\frac{d \theta_{s}}{d s}=\frac{F_{s}}{M c^{2} \beta^{2} \gamma}
\end{array}\right.
$$

where $F_{x, z, s}$ are the force components in the laboratory reference frame.

The force acting on the ion inside the cooling section is the sum of Lorenz force from solenoid magnetic field, the electron beam space charge force, the friction force and the force randomly distributed around zero value, which determines the diffusion in the electron beam. The influence of the electron beam space charge and longitudinal magnetic field on the ion motion can be described in a standard way and here we will discuss only calculation of the friction force.

The friction force components are calculated in PRF using the standard list of parameters. Model of electron beam has to transform the ion velocity components to PRF and calculate all the parameters required for the friction force calculation. After calculation of the friction force in PRF the electron beam model calculates the force components in LRF that can be used in the right hand side of the system (4.2).

In presence of transverse components of the guiding magnetic field at the axis of the cooler solenoid the electron beam is characterized by co-ordinates of its center and angle between electron beam axis and ion equilibrium orbit. In this case the ion transverse angles in the frame referenced to the 
electron beam orbit have to be corrected by the values of the angular misalignment $\delta \theta$ between electron and ion beams:

$$
\theta_{\alpha}^{*}=\theta_{\alpha}-\delta \theta_{\alpha}(s), \alpha=x, z \text {, }
$$

where $\theta^{*}$ is the ion angle in the electron beam reference frame.

The space charge of the electron beam determines the electron average velocity and electron velocity spread as functions of co-ordinates inside the electron beam. Electron beam density in a general case is also a function of co-ordinates. To calculate the ion position inside the electron beam one needs to introduce transverse co-ordinates of the electron bunch center $\left(x_{c}, z_{c}\right)$ and distance between the centers of the electron and ion bunches $s_{c}$. Under assumption that $\delta \theta \cdot l_{\text {bunch }}<<a$ the ion co-ordinates inside the electron beam are:

$$
x^{*}=x+x_{c}, z^{*}=z+z_{c},\left(s-s_{0}\right)^{*}=\left(s-s_{0}\right)+s_{c}
$$

The transformation of the ion co-ordinates in accordance with the magnetic field line curvature, choice of the numerical algorithm for integration of the system (4.2) and calculation of the particle loss probability are provided by the model of the cooler. The particle loss probability is calculated by integration of expression (3.39):

$$
P_{\text {loss }}=\int_{0}^{l_{c o o l}} \frac{\alpha_{r} n_{e}}{\gamma^{2} \beta c} d s,
$$

which is performed along the particle trajectory during numerical integration of the ion motion equations.

The model of the cooler is realized as a procedure which obtains at the entrance the $6 \mathrm{D}$ vector of initial particle co-ordinates, transforms components of this vector in accordance with solution of the system (4.2) and returns the value of the ion loss probability.

All stages of the algorithm are presented in the Fig. 4.1. 


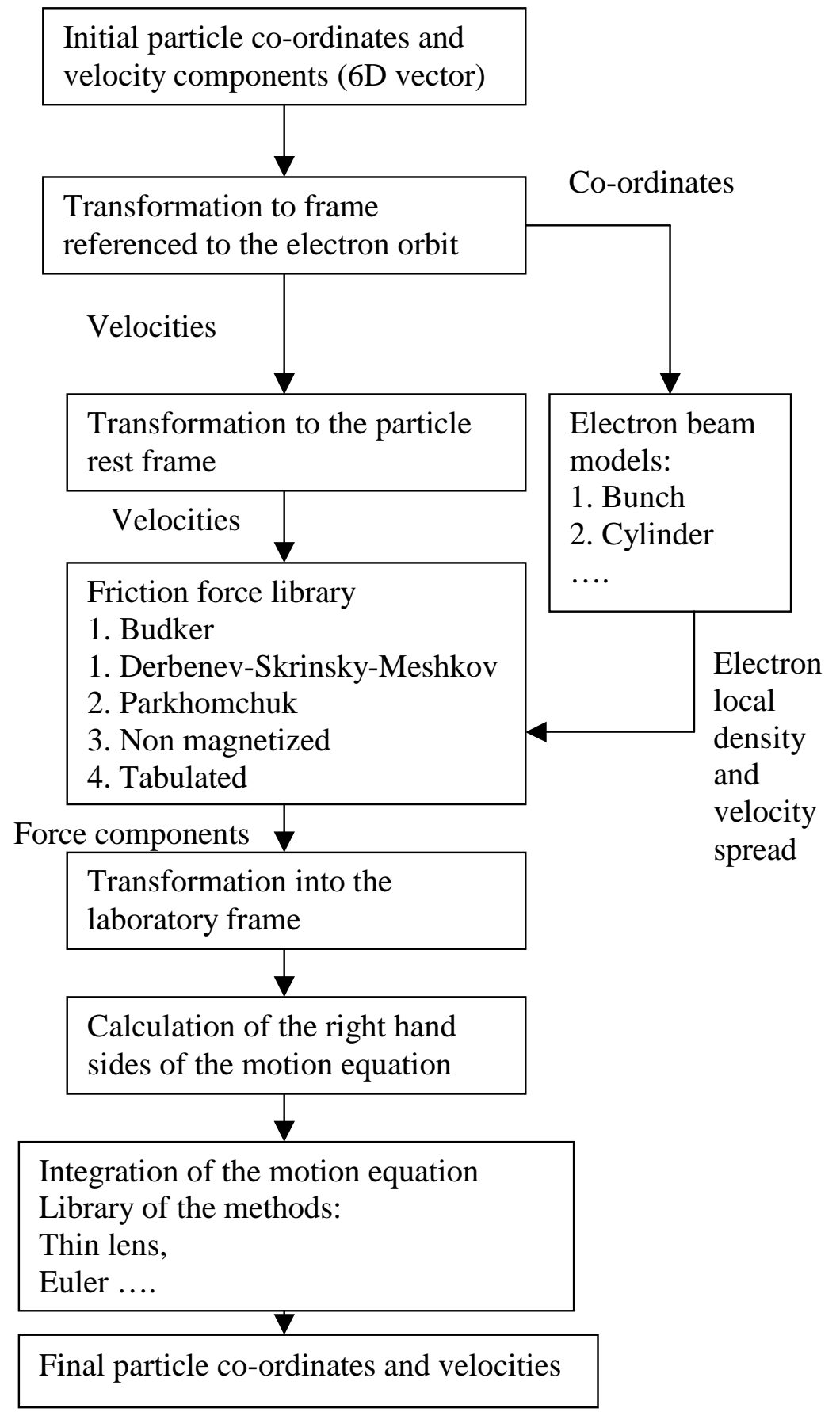

Fig. 4.1. Block scheme of the particle propagation through the cooling section.

\subsection{Models of electron beam}

The model of electron beam calculates list of parameters required for friction force procedure, transforms the ion velocity from LRF to PRF, makes a choice of the friction force procedure from library and transforms the force components from PRF to LRF. These parameters depend on the electron beam model:

the local density of electrons,

the electron velocity spread in transverse plane,

the electron longitudinal velocity spread. 
The local electron beam density is determined by geometry of the electron beam. The local velocity spread is the function of co-ordinates inside the electron beam if the space charge effects are taken into account. The space charge effects can also lead to shift of the electron mean velocity. The last effect does not change the beam velocity spread and it is taken into account by required correction of the longitudinal component of the ion velocity.

In the present version of the program various models of the electron beam are realized:

1. Uniform round cylinder,

2. Gaussian bunch of round (or elliptical) cross-section,

3. Uniform bunch,

4. Cylinder with round (or elliptical) cross-section with Gaussian distribution in transverse plane.

5. Hollow beam

6. Electron Array

The first model corresponds to electron beam of usual electron cooling system. For this model the input parameters are electron beam current and radius and electron beam density is assumed to be independent on the ion co-ordinates inside the electron beam. The local electron beam density in LRF is constant determined by the expression:

$$
n_{e}=I_{e} /\left(e \pi a^{2} \beta c\right)
$$

For this model the space charge effects in the electron beam are taken into account as described in the Chapter 4.2.

Input parameters for the Gaussian bunch model are the following:

- r.m.s. bunch dimensions,

- offset between electron and ion bunch centres,

- number of electrons in the bunch $N_{e}$.

For this model the local electron density in PRF in the point $\left(x, z, s-s_{0}\right)$ is calculated as follows:

$$
n_{e}=\frac{N_{e}}{(2 \pi)^{3 / 2} \sigma_{x} \sigma_{z} \sigma_{s} \gamma} \exp \left(-\frac{x^{2}}{2 \sigma_{x}^{2}}-\frac{z^{2}}{2 \sigma_{z}^{2}}-\frac{\left(s-s_{0}\right)^{2}}{2 \sigma_{s}^{2}}\right)
$$

where $\sigma_{x} \sigma_{z}$ are r.m.s. transverse bunch dimensions, $s-s_{0}$ is calculated taking into account offset between electron and ion bunch centers.

As an output parameter the program calculates electron peak current as:

$$
I_{e}=\frac{e N_{e} \beta c}{\sigma_{s} \sqrt{2 \pi}},
$$

where $\sigma_{s}$ is r.m.s. bunch length.

The Uniform bunch model presumes that the electron bunch has a uniform density in transverse direction and Gaussian distribution along longitudinal co-ordinate. Correspondingly the input parameters for are the following:

- the bunch transverse dimensions,

- the rms bunch length, 
- offset between electron and ion bunch centres,

- number of electrons in the bunch $N_{e}$.

For this model the local electron density in PRF in the point $\left(s-s_{0}\right)$ is determined as follows:

$$
n_{e}=\frac{N_{e}}{(2 \pi)^{1 / 2} \pi a_{x} a_{z} \sigma_{s} \gamma} \exp \left(-\frac{\left(s-s_{0}\right)^{2}}{2 \sigma_{s}^{2}}\right) \text {, }
$$

where $a_{x} a_{z}$ are the transverse bunch dimensions, $s-s_{0}$ is calculated taking into account offset between electron and ion bunch centers.

As an output parameter the program calculates electron peak current in accordance with the formula (4.8).

The model of Gaussian cylinder can be used for cooling time calculation in the case when short electron bunch moves forward and back along a long ion bunch during the time shorter than cooling time. This model is more realistic in the case of electron cooling with magnetized circulating electron beam. The model input parameters are:

- r.m.s. dimensions of the cylinder cross-section,

- number of electrons per unit of length $\lambda_{e}$.

Local electron beam density in PRF in a position $(x, z)$ is calculated in accordance with:

$$
n_{e}=\frac{\lambda_{e}}{2 \pi \sigma_{x} \sigma_{z} \gamma} \exp \left(-\frac{x^{2}}{2 \sigma_{x}^{2}}-\frac{z^{2}}{2 \sigma_{z}^{2}}\right) \text {. }
$$

Electron beam current for this model is given by the formula:

$$
I_{e}=e \lambda_{e} \beta c .
$$

The last two models of the electron beam do not take into account the space charge effects in the electron beam. However, the expressions for the self-fields of the electron beam are introduced into the program as described in the next chapter and can be introduced into calculation if necessary.

The model Electron Array is the most recent and general model of electron beam representation. In this model the 6-D distribution of electron beam is generated by some other code. This distribution can be read into the BETACOOL. In this case, the friction force can be calculated for an arbitrary distribution of electrons.

\subsection{Model of the cooler}

The model of electron cooler provides a choice of the numerical integration for the system (4.2) and takes into account displacements of the electron beam position in the cooling section. The system can be solved by assuming two different models of the cooler:

1. Electron cooler as a thin lens,

2. Electron cooler as a system of non-zero length. 
In the case, when the friction force variation along the cooling section is negligible and relative change of the particle momentum is small, the electron cooling section can be treated as a thin lens. This model presumes also that the ion transverse co-ordinates do not change during motion inside the cooler. Thus, the ion angle variation is calculated as following

$$
\Delta \theta=\frac{F}{M c^{2} \beta^{2} \gamma} l_{\text {cool }},
$$

where $l_{\text {cool }}$ is the cooling section length, $F$ is the friction force in LRF. The ion co-ordinates are not changed inside the cooler.

For the non-zero length of the cooler the ion motion equation can be solved using one of the numerical methods: Euler or Runge-Kutta. Numerical integration of the ion motion equations is necessary also in the case, when electron beam trajectory does not coincide with the ion equilibrium orbit.

One of the options in the program is to introduce the electron beam trajectory position from an external file. In this file the transverse coordinates of the electron trajectory are specified in a few points along the cooling section. In this case, the cooling section is divided by a few sub intervals in longitudinal direction. In each interval the electron trajectory assumed to be a straight line displaced from the ion trajectory and having some angle with it. Position of the electron beam trajectory is determined by its transverse co-ordinates at the entrance and at the exit of the sub interval.

The ion transverse co-ordinates relatively to the electron beam trajectory are calculated as functions of its longitudinal co-ordinate (independent variable in the system 4.2) in accordance with the angles between electron and ion beam axis:

$$
\theta_{e, i, x}=\frac{x_{f}-x_{0}}{l_{S I}}, \theta_{e, i, z}=\frac{z_{f}-z_{0}}{l_{S I}},
$$

where $x_{f}, x_{0}$ are initial and final horizontal co-ordinates of the electron beam trajectory in the sub interval correspondingly, calculated from the ion equilibrium orbit. $l_{S I}$ is the length of corresponding sub interval. The same is for vertical position. The ion velocity components in the frame referenced to the electron beam trajectory are corrected by these angles. We assume that the angles between the electron beam trajectory and the ion equilibrium orbit are sufficiently less than unit. In this case correction of the ion longitudinal velocity is not necessary.

Under assumption that the both angles are sufficiently less than the ion angle the ion co-ordinates in the sub interval are calculated as:

$$
\begin{gathered}
x=x+x_{0}+\theta_{e, i, x} s \\
z=z+z_{0}+\theta_{e, i, z} s \\
\theta_{x}=\theta_{x}+\theta_{e, i, x} \\
\theta_{z}=\theta_{z}+\theta_{e, i, z},
\end{gathered}
$$

where co-ordinate $s$ is calculated from the entrance of corresponding sub interval. 


\section{Kick of the ion momentum components in the electron cooling section}

The Model Beam algorithm presumes that the ion geometrical co-ordinates do not change after crossing the cooling section and action of the cooling leads to change of the ion momentum components only (the thin lens approximation). The kick in the ion beam momentum after crossing the cooler is calculated on the basis of the map of the cooling section. The map transforms the initial ion co-ordinates in $6 \mathrm{D}$ phase space to the final ones:

$$
\vec{X}_{i n}=\left\{x_{i n}, \theta_{x, i n}, z_{i n}, \theta_{z, i n},\left(s-s_{0}\right)_{i n}, \theta_{s, i n}\right\}->\vec{X}_{f}=\left\{x_{f}, \theta_{x, f}, z_{f}, \theta_{z, f},\left(s-s_{0}\right)_{f}, \theta_{s, f}\right\} \text {, }
$$

The Model Beam algorithm ignores the ion geometry co-ordinate variation $\left(x, z, s-s_{0}\right)$ and multiplies the ion momentum components by the factor:

$$
\theta_{\xi}=\theta_{\xi, 0} \times \exp \left(\left(\theta_{\xi, f}-\theta_{\xi, i n}\right) \frac{\Delta t}{T_{r e v}}\right)
$$

where $\xi=x, z, s-s_{0}, \Delta t-$ step over time in the dynamics simulation.

The particle losses after crossing the cooler are calculated separately for total beam and for Model Beam. In the total beam the ion number is decreased in accordance with:

$$
N=N_{0} \times \exp \left(-\frac{P_{l o s s}}{N_{M B}} \frac{\Delta t}{T_{r e v}}\right)
$$

where $P_{\text {loss }}$ is the particle loss probability calculated by the map of the cooling section in accordance with (4.5), $N_{M B}$ is the particle number in the Model Beam. For the particle in the Model Beam the program generates random number uniformly distributed in the range from 0 to 1 , if this number is smaller than $P_{\text {loss }}$ for this particle new co-ordinates are generated in accordance with current ion distribution as it described in the Chapter 2. If the random number is larger than $P_{\text {loss }}$ the particle is alive and its co-ordinates are not changed.

\section{Algorithm of the electron cooling time calculation}

For rms dynamics simulation one needs to calculate characteristic cooling times and the lifetime due to recombination in the cooling section. In the present version of the program the cooling times can be calculated for two models of the ion beam. In the frame of the first model ("single particle") the ion beam is presented by an ion having invariants of the motion corresponded to r.m.s. beam emittances. In this case a change of the ion motion invariants after crossing the cooling section is averaged over the phases of the ion betatron and synchrotron oscillations in the ring. In the second model ("Monte-Carlo") the ion beam is presented as an array of particles. The particle distribution over co-ordinates is Gaussian at corresponding r.m.s. parameters and matched with the lattice parameters of the ring in the cooling section. The beam is propagated through the cooling section particle by particle and r.m.s. parameters of the particle distribution function at the exit of the cooler are used for the cooling time calculation. 


\subsection{Calculation of the characteristic times of the ion motion invariants}

The ion co-ordinates at the entrance of the cooling section can be expressed as functions of the motion invariants and phases of the betatron and synchrotron oscillations. The ion "betatron" coordinates and momentum inside the cooling section can be calculated in accordance with

$$
x_{\beta}=\sqrt{I_{x} \beta_{x}} \sin \varphi, \quad x_{\beta}^{\prime}=\sqrt{\frac{I_{x}}{\beta_{x}}}\left(\cos \varphi+\alpha_{x} \sin \varphi\right) .
$$

where $\varphi$ - is the phase of the horizontal betatron oscillation, $\alpha_{x}$ and $\beta_{x}$ are alpha and beta functions at the entrance of the cooling section. The same expressions are used for $z$ co-ordinate with substitution of corresponding alpha and beta function values, where we introduced the notation $x^{\prime}=\frac{p_{x}}{p}$. For r.m.s. particle the mean square of the co-ordinates have to be equal corresponding standard deviations:

$$
\sigma_{x}=\sqrt{\varepsilon_{x} \beta_{x}}, \sigma_{x^{\prime}}=\sqrt{\varepsilon_{x} \gamma_{x}},
$$

where $\varepsilon_{x}$ is r.m.s. emittance and gamma function is calculated as usual $\gamma_{x}=\frac{1+\alpha_{x}^{2}}{\beta_{x}}$. To satisfy this equation the invariant of the motion $I_{x}$ is to be equal to two sigma emittance $I_{x}=2 \varepsilon_{x}$.

Longitudinal emittance of the ion beam is determined in the program as a mean square of the particle momentum deviation:

$$
\varepsilon_{l}=\left\langle\delta^{2}\right\rangle
$$

Here to simplify the notation the ion relative longitudinal momentum deviation is denoted as $\delta=\frac{\Delta p}{p}$. In the case of coasting beam the cooling time is calculated by averaging over two values of the momentum deviation:

$$
\delta= \pm \sqrt{\varepsilon_{l}} .
$$

For the bunched ion beam one can express the particle momentum deviation and its longitudinal coordinate (distance from the bunch center) as a function of the phase of synchrotron oscillations:

$$
\delta=\sqrt{I_{l}} \cos \psi, s-s_{0}=\beta_{l} \sqrt{I_{l}} \sin \psi
$$

here the "synchrotron function" is determined as:

$$
\beta_{l}=\frac{R|\eta|}{Q_{s}},
$$

where $R$ is the mean ring radius, $\eta$ - off momentum factor, $Q_{s}$ is the synchrotron tune. The standard deviations for longitudinal degree of freedom are calculated in accordance with the longitudinal emittance definition: 


$$
\sigma_{\delta}=\sqrt{\varepsilon_{l}}, \sigma_{s}=\beta_{l} \sigma_{\delta}
$$

and as in the case of transverse motion to satisfy this equation the invariant of the motion $I_{l}$ is equal to two sigma emittance $I_{l}=2 \varepsilon_{l}$.

The total values of the ion $x$-co-ordinates are equal to:

$$
x=x_{\beta}+D_{x} \delta, x^{\prime}=x_{\beta}^{\prime}+D_{x}^{\prime} \delta,
$$

where $D$ and $D^{\prime}$ are dispersion and its derivative in the cooling section. For the vertical plane all the procedures are provided by the same way.

Using these formulae at given values of the phases of betatron and synchrotron oscillations one can calculate particle co-ordinates and velocity components at the entrance of the cooling section. The map of the electron cooling section transforms the ion coordinates to the exit of the cooler and returns the probability of the ion loss. New values of the particle motion invariants are calculated in accordance with the following formulae. Invariant of the particle longitudinal motion for a coasting beam is calculated simply as a square of the relative momentum deviation, for a bunched beam

$$
I_{l}=\left(\delta^{2}+\left(\frac{\left(s-s_{0}\right)}{\beta_{l}}\right)^{2}\right) .
$$

The Courant - Snider invariants of the particle betatron motion are calculated from the "betatron" particle co-ordinates:

$$
x_{\beta}=x-D_{x} \delta, x_{\beta}^{\prime}=x^{\prime}-D_{x}^{\prime} \delta .
$$

The invariant calculated in accordance with

$$
I_{x}=\left(\beta_{x} x_{\beta}^{\prime 2}+2 \alpha_{x} x_{\beta} x_{\beta}^{\prime}+\gamma_{x} x_{\beta}^{2}\right)
$$

corresponds to 2-sigma emittance of the beam. The invariant of vertical motion is calculated with substitution $z$ instead $x$.

When the electron cooler is treated as a thin lens, the lattice parameters at the exit of the cooler coincide with that ones at the entrance. For the cooler model taking into account finite length of the cooling section the tracking of the lattice parameters through the cooler is necessary. Neglecting the influence of the cooler magnetic field on the ion motion the lattice parameters at the exit of the cooling section are calculated as:

$$
\beta_{f, x}=\beta_{x}-2 \alpha_{x} l_{\text {cool }}+\gamma_{x} l_{\text {cool }}^{2}, \alpha_{f, x}=\alpha_{x}-\gamma_{x} l_{\text {cool }} .
$$

The characteristic time of change of the rms particle invariant is calculated in accordance with:

$$
\frac{1}{\tau_{\text {cool }}}=\frac{1}{I} \frac{\langle\delta I\rangle}{T_{\text {rev }}}
$$

where $T_{\text {rev }}$ is the particle revolution period in the storage ring, the brackets mean averaging over the phases of betatron and synchrotron oscillations: 


$$
\langle\vec{I}\rangle=\frac{1}{8 \pi^{3}} \iint_{0}^{2 \pi} \int \delta \vec{I}\left(\vec{I}, \varphi_{x}, \varphi_{z}, \varphi_{s}\right) d \varphi_{x} d \varphi_{z} d \varphi_{s} .
$$

The formula (6.13) gives the cooling time for single particle, which invariants of the motion correspond to the beam r.m.s. emittances.

The ion beam life time is calculated by averaging over the phases the ion loss probability:

$$
\frac{1}{\tau_{\text {life }}}=\frac{\left\langle P_{\text {loss }}\right\rangle}{T_{\text {rev }}},
$$

where the averaging is performed by the same way as for invariants:

$$
\left\langle P_{\text {loss }}\right\rangle=\frac{1}{8 \pi^{3}} \iint_{0}^{2 \pi} \int P_{\text {loss }}\left(\vec{I}, \varphi_{x}, \varphi_{z}, \varphi_{s}\right) d \varphi_{x} d \varphi_{z} d \varphi_{s} .
$$

\subsection{Calculation of the characteristic times of emittance variation using Monte Carlo method}

Calculation of the characteristic times of the beam emittance variation is performed in the program using Monte Carlo method, which includes the following steps.

1. Generation of the beam as an array of the particles matched with the optics structure of the ring at the entrance of the cooler.

2. Propagation of the beam through the cooler particle by particle using the map of the cooling section.

3. Calculation of the new values of the beam emittances after crossing the cooler.

4. Calculation of the cooling time in accordance with the formula:

$$
\frac{1}{\tau_{\text {cool }}}=\frac{1}{\varepsilon} \frac{\Delta \varepsilon}{T_{\text {rev }}} .
$$

The beam generation is performed as described in the Chapter 2. For the beam emittance calculation one can use one of the procedures described in the Chapter 2. The result of the calculation can slightly depends on the procedure used for the emittance calculation. This fact can be illustrated on example of simplest model of the cooler. One can treat the cooling section as a thin lens - in this case the particle co-ordinates keep their initial values and components of momentum are changed in accordance with the friction force value. Therefore the beam Twiss parameters are changed in the same time when the lattice parameters are constant due to zero length of the cooler according to this model. This leads to the mismatch of the beam with the ring optics structure and, as a result, to an additional emittance growth if the emittance is calculated through rms co-ordinates of the beam.

When the electron cooler is treated as a system of non-zero length the program provides tracking of the lattice parameters through the cooling section as in the case of single particle cooling time calculation.

The ion beam lifetime is calculated with the same formula as in this case of single particle model:

$$
\frac{1}{\tau_{\text {life }}}=\frac{\left\langle P_{\text {loss }}\right\rangle}{T_{\text {rev }}},
$$


however, here, the averaging of the loss probability is provided over the particles.

\section{References}

[1] G. Budker, Atomnaya Energia, V. 22, p. 346 (1967).

[2] S. Chandrasekhar, Principles of Stellar Dynamics (U. Chicago Press, 1942).

[3] J. J. Binney, MNRAS 181, p. 735 (1977).

[4] I. Meshkov, Electron cooling: Status and Perspectives, Phys. Part. Nucl., 25, (6), 1994, 631;

Physics and Technique of Electron Cooling, RIKEN-AF-AC-2, 1997.

[5] Ya.S. Derbenev and A.N. Skrinsky, Fyzika plasmy, 1978. v.4, p.492; Part. Accelerators 8, p. 235 (1978).

[6] D.V. Pestrikov, BINP Preprint 2002-57 (Novosibirsk, Russia, 2002).

[7] V. Parkhomchuk, New insights in the theory of electron cooling, NIM A 441 (2000) 9-17, p.9

[8] A. Fedotov, D. Bruhwiler, A. Sidorin, D. Abell, I. Ben-Zvi, R. Busby, J. Cary, V. Litvinenko, Physical Review ST Accel. Beams, V. 9, 074401 (2006).

[9] A. Wolf et al., Recombination in electron coolers, NIM A 441(2000), 183

[10] A. Sidorin, I. Meshkov, T. Katayama, P. Zenkevich "Ion Bunch Stability in the Double Storage Ring", RIKEN-AF-AC-19, (February 2000).

[11] Ya. Derbenev, "Electron cooling of heavy ions in solenoid with undulator", TJNAL, 13.02.2001, unpublished.

[12] M. Bell, J.S. Bell, Capture of cooling electrons by cool protons, Particle accelerators, 1982 Vol.12 pp.49-52.

[13] C. Toepffer, Phys. Rev. A 66, 022714 (2002).

[14] V. Parkhomchuk, Russian Doctorate Dissertation (BINP, Novosibirsk, 1984); also in Proceedings of ECOOL'84.

[15] Electron cooling at RHIC: http://www.bnl.gov/cad/ecooling

[16] N.S. Dikansky, V.I. Kudelainen, V.A. Lebedev, I.N. Meshkov, V.V. Parkhomchuk, A.A. Sery, A.N. Skrinsky, B.N. Sukhina, "Ultimate possibilities of electron cooling", BINP Preprint 198861 (Novosibirsk). 


\section{Part III. Intrabeam scattering}

\section{Introduction}

Intrabeam scattering (IBS) in the ion beam causes two processes: relaxation of the beam to a thermal equilibrium between degrees of freedom and diffusion growth of $6 \mathrm{D}$ phase volume of the beam due to variation of lattice parameters along ring circumference.

For Gaussian distribution of ions over velocity, four models for IBS calculation are realized within the BETACOOL: - Piwinski [1], Martini (extended Piwinski) [2], Bjorken-Mtingwa [3] and Jie Wei [4] models. The Martini model does not require additional assumptions for calculation of the beam emittance growth times. Piwinski's model can be deduced from Martini's model neglecting a variation of dispersion and beta function along the ring orbit. In the model proposed by Jie Wei characteristic times of emittance variation are calculated for real lattice parameters of the ring under a few additional assumptions, which correspond to storage rings with ion energy higher than the transition energy (for example, RHIC operation at collision energy). The description of the models is presented in following Sections in accordance with the notation of the original papers [1 -4$]$.

For non-Gaussian distributions, a variety of approximate models were developed which are described in Section 5.2. A more rigorous treatment of IBS for an arbitrary distribution function via diffusion and friction coefficients is presently under development.

\section{Structure of the effect}

Simulation of the intrabeam scattering (IBS) process is based on calculation of the particle momentum variation due to coulomb interactions with other particles of the beam. In the BETACOOL the particle momentum variation can be calculated using analytical expressions for diffusion coefficients or, for coasting ion beam, using Molecular Dynamics (MD) technique. On the basis of both approaches each optic element of the ring is presented as a map for IBS process.

The map of the IBS process based on direct calculation of the ion coulomb interaction can be combined with the transformation map of optic element calculated from external focusing fields. In such a form the IBS process is simulated in the frame of tracking algorithm based on MD technique. This algorithm uses as input parameters the particle array presenting the ion beam and characteristics of external focusing fields.

The map of IBS process based on analytical theory calculates the growth rates for three degrees of freedom by numerical evaluation of integrals over ion distribution function assuming that the distribution function has Gaussian shape. The values of the growth rates are used for calculation of the individual particle momentum variation. The calculation of the growth rates requires as input parameters the rms beam emittance and lattice functions of the ring in given optic element.

In the frame of rms dynamics simulation the growth rates calculated in each optic element are averaged over the ring circumference. This procedure requires as input parameters the rms beam emittances and description of the ring optic structure. The ring optic structure can be presented in both variants: as a specification of the optic elements or as dependencies of the lattice functions on the longitudinal co-ordinate along the ring circumference. In the first case the program initially provides tracking of Twiss parameters along the ring and transforms the ring model to the form of the lattice functions. 
In the frame of a few models for the IBS growth rates calculation one needs mean parameters of the ring only. In this case detail description of the ring optic structure is not necessary and the IBS process can not be presented by a transformation map. Such models of the IBS can be used only in Model Beam algorithm and rms dynamics simulation.

For Model Beam algorithm a few "detailed" models of the IBS process were developed. In the "detailed" models the kick of the particle momentum components is calculated as a function of the particle co-ordinates in 6D phase space.

\section{Models of IBS}

When particles in a beam scatter within each other one needs to consider both large and small angle scattering. The effect when particles can be lost as a result of a single collision event (large-angle scattering) is called Touschek effect. When the scattering angles are small, random addition of such small scattering events can lead to a growth of beam dimensions. Such a multiple Coulomb scattering was first applied to explain emittance growth in electron beams (Bruck, Le Duff) and was called "multiple Touschek effect".

Typically, in electron machines the longitudinal beam temperature is much smaller than the transverse, an assumption which was used in original studies of the Touschek effect. Multiple Coulomb scattering was later generalized by Piwinski for proton machines without making any restrictions on the magnitude of beam temperatures, thus making it possible to transfer energy from the longitudinal into transverse via collisions. The generalized treatment of multiple small-angle Coulomb scattering was then renamed as the Intrabeam Scattering (IBS). The IBS theory was later extended to include variations of the betatron functions and momentum dispersion function along the lattice, and was summarized by Martini (referred in this report as Martini's model).

The different approach to IBS using the scattering matrix formalism from quantum electrodynamics was used by Bjorken and Mtingwa (B-M model). Both B-M and Martini's models are in good agreement with one another. In what way IBS in particle beams is different from similar scattering of gas molecules? In circular accelerators, the curvature of the orbit produces a dispersion. Because of the dispersion, a change of energy leads to change in the betatron amplitude. In other words, we have coupling of the longitudinal and transverse motion.

Another consequence of this curvature effect is the negative-mass behavior of particles so that conservation law of beam temperature leads to a simple conclusion that below transition energy one can have an equilibrium between the transverse and longitudinal temperatures while above transition (in the "negative-mass" regime) there is a continuous emittance increase in both transverse and longitudinal directions. Is this coupling effect always important in circular accelerator and one always needs to use standard IBS approach rather than the gas-relaxation formula? This question was studied in detail by Sorensen. He found that the IBS growth rates can be simply represented by a universal curve. When plotted against parameter $\varepsilon /(\Delta p / p)^{2}$ (where $\varepsilon$ in the rms beam emittance) such a universal curve shows a minimum ("brake-up") point. To the left of such a minimum the transverse growth rate dominates, while to the right - the growth rate is dominated by the longitudinal growth rate. This was explained by a collapse of the longitudinal velocity distribution with energy. The longitudinal and transverse velocities spread in the beam rest frame are given by:

$$
\Delta v_{\|}=\frac{\Delta p}{\gamma m}, \quad \Delta v_{x}=\frac{1}{m} \sqrt{\frac{\varepsilon}{\beta_{x}}} p,
$$


which gives

$$
\frac{\varepsilon}{(\Delta p / p)^{2}}=\frac{\varepsilon}{\gamma^{2}}
$$

As a result, for a high energy $\gamma>\gamma_{t r}$, the effect of collapsed velocity distribution dominates over coupling, and the longitudinal IBS can be described by the gas-relaxation formula, independent of the ring lattice (with some degree of accuracy).

\subsection{Analytical model of the multiple particle collisions}

According to Piwinski [1], the relative changes of momenta after a collision between two particles labeled by 1 and 2 are (for the longitudinal and radial axis):

$$
\begin{gathered}
\frac{\delta p}{p}=\frac{1}{2}(2 \alpha \gamma \sin \bar{\psi} \sin \bar{\phi}+\gamma \xi(\cos \psi-1)) \\
\frac{\delta p_{x}}{p}=\frac{1}{2}\left(\left[\varsigma \sqrt{\left.\left.1+\frac{\xi^{2}}{4 \alpha^{2}} \cos \phi-\frac{\xi \theta}{2 \alpha} \sin \phi\right] \sin \psi+\theta(\cos \psi-1)\right)},\right.\right.
\end{gathered}
$$

with the notation

$$
\gamma \xi=\frac{\Delta p_{1}}{p}-\frac{\Delta p_{2}}{p}, \theta=x_{1}^{\prime}-x_{2}^{\prime}, \varsigma=z_{1}^{\prime}-z_{2}^{\prime}
$$

$\gamma$ - is the particle Lorenz factor.

$\bar{\psi}$ and $\bar{\phi}$ denote the scattering axial and azimuthal angles in the center of mass (C.M.) between the particles after the collision.

$2 \alpha \ll 1$ is the angle between the momentum vectors of the colliding particles in the laboratory reference frame; this angle can be evaluated by taking the scalar product of the particle momentum vectors (Fig. 3.1).

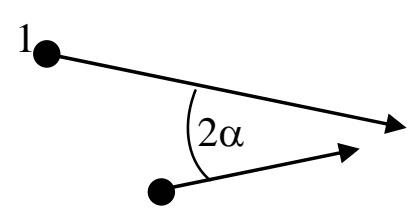

a

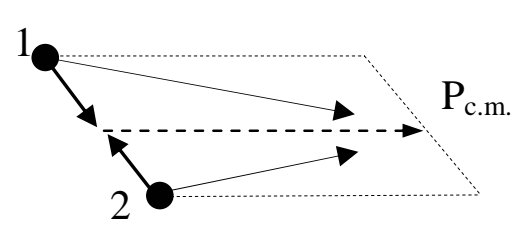

b

Fig. 3.1. Two particles collision scheme (non-relativistic case)
a) - LRF;
b) C.M. reference frame

The change of particle momentum after collision leads to the corresponding change of the particle motion invariant. It can be calculated under assumption that the radial position of the particle is not changed during the interaction, which is sufficiently short.

The radial displacement of the particle from the closed orbit is equal to the sum of the betatron and momentum change contributions, which is, to the first order in $\Delta p / p$, described as: 


$$
x=x_{\beta}+D(\Delta p / p)
$$

where $D(\mathrm{~s})$ is the dispersion function. The derivative of $x$ with respect to the co-ordinate $s$ along the equilibrium orbit is equal to the small angle the particle makes with $s$-axis:

$$
x^{\prime} \equiv \frac{P_{x}}{P}=x_{\beta}^{\prime}+D^{\prime}\left(\frac{\Delta p}{p}\right)
$$

where $p_{x}$ is the $\mathrm{x}$-component of the momentum.

The Courant and Snyder invariant of a particle transverse motion is described as the following:

$$
I_{x}=\gamma_{x} x_{\beta}^{2}+2 \alpha_{x} x_{\beta} x_{\beta}^{\prime}+\beta_{x} x_{\beta}^{\prime 2},
$$

where $\beta_{x} \gamma_{x}-\alpha_{x}^{2}=1$ and $\beta_{x}^{\prime}=-2 \alpha_{x}$.

The change of the Courant and Snyder invariant $I$ is given by:

$$
\beta_{x} \delta I_{x}=\left(1+\alpha_{x}^{2}\right)\left(2 x_{\beta} \delta x_{\beta}+\delta x_{\beta}^{2}\right)+2 \alpha_{x} \beta_{x}\left(x_{\beta}^{\prime} \delta x_{\beta}+x_{\beta} \delta x_{\beta}^{\prime}+\delta x_{\beta} \delta x_{\beta}^{\prime}\right)+\beta_{x}^{2}\left(2 x_{\beta}^{\prime} \delta x_{\beta}^{\prime}+\delta x_{\beta}^{\prime 2}\right) .
$$

In accordance with the assumption $x=$ constant one finds:

$$
\delta x_{\beta}=-D(\delta p / p), \delta x_{\beta}^{\prime}=\left(\delta p_{x} / p\right)-D^{\prime}(\delta p / p)
$$

since $\delta \Delta p / p=\delta p / p$, where $\delta \mathrm{p}$ is the full particle momentum change in the collision, it means that variation of the longitudinal component of the particle momentum leads to a change of the horizontal motion invariant, when dispersion is not equal to zero:

$$
\begin{gathered}
\frac{\delta I_{x}}{\beta_{x}}=-\frac{2}{\beta_{x}}\left(x_{\beta}\left(\frac{\left(1+\alpha_{x}^{2}\right)}{\beta_{x}} D+\alpha_{x} D^{\prime}\right)+x_{\beta}^{\prime} \widetilde{D}\right) \frac{\delta p}{p}+\frac{\left(D^{2}+\widetilde{D}^{2}\right)}{\beta_{x}^{2}}\left(\frac{\delta p}{p}\right)^{2}+2\left(x_{\beta}^{\prime}+\frac{\alpha_{x}}{\beta_{x}} x_{\beta}\right) \frac{\delta p_{x}}{p}+ \\
+\left(\frac{\delta p_{x}}{p}\right)^{2}-\frac{2 \widetilde{D}}{\beta_{x}} \frac{\delta p}{p} \frac{\delta p_{x}}{p}
\end{gathered}
$$

where $\tilde{D}=\alpha_{x} D+\beta_{x} D^{\prime}$. The changes of vertical and longitudinal invariants are equal:

$$
\begin{gathered}
\delta I_{z}=2\left(\beta_{z} \theta_{z, \beta}+\alpha_{x} z_{\beta}\right) \frac{\delta p_{z}}{p}+\beta_{z}\left(\frac{\delta p_{z}}{p}\right)^{2}, \\
\delta I_{s}=2 m \frac{\delta p}{p} \frac{\Delta p}{p}+m\left(\frac{\delta p}{p}\right)^{2}
\end{gathered}
$$

Here we assume that $D$ and $D^{\prime}$ have zero value in the vertical plane. In this case $z_{\beta}=z$.

The dependence of the horizontal invariant changes on a longitudinal momentum change leads to heating of the ion beam due to the multiple particle collisions: the particle momentum components are changed in accordance with momentum conservation law, however in the presence of dispersion 
the sum of the particle motion invariants can increase or decrease depending on collision parameters. Therefore in the frame of this model, IBS in a linear transport line leads only to relaxation between degrees of freedom of the ion beam, where the six dimensional phase space is constant (the energy source producing the heating is large value of the longitudinal component of the ion momentum in LRF). In cycling accelerators IBS leads to two effects: relaxation between degrees of freedom and change of six dimensional phase space volume of the beam in presence of dispersion.

The variation of the beam phase space volume can be calculated by averaging of the particle invariant change over the collisions in accordance with the particle distribution function. A.Piwinski introduces the time derivative in the C.M. system of the average radial emittance for all particles (denoted $\langle\varepsilon\rangle$ ) by means of the integral:

$$
\left\langle\frac{d}{d t} \frac{\langle\varepsilon\rangle}{\beta_{x}}\right\rangle=\int 2 c \beta P \int_{\psi_{m}}^{\pi} d \psi \int_{0}^{2 \pi} d \phi \frac{d \sigma}{d \Omega} \frac{\delta I_{1}}{\beta_{x}} \sin \psi d \tau
$$

where the outer brackets $\langle\ldots\rangle$ indicate the average value around the ring. The first integral extends over all phase space betatron co-ordinates, momentum spread values and azimuthal location of two interacting particles (denoted 1 and 2). $P$ is the probability density function for the betatron amplitudes and angles, the momentum errors and the azimuthal positions of the interacting particles. $\bar{P}$ is the probability written in terms of the C.M variables, while $P$ is the same probability written for the laboratory frame variables. Henceforth the bar indicates the values in the C.M. reference frame. $d \sigma / d \Omega$ is the differential cross-section in the C.M. system for the scattering into the element of solid angle $d \bar{\Omega}$ at given angles $\bar{\psi}$ and $\bar{\phi} . d \bar{t}$ is the C.M time interval which is related to the laboratory time by the equation $d t=\gamma \cdot d \bar{t}$. P may be written according to independent probability density law:

$$
P=\rho_{X_{\beta} X_{\beta}^{\prime}}\left(x_{\beta_{1}} x_{\beta_{1}}^{\prime}\right) \cdot \rho_{X_{\beta} X_{\beta}^{\prime}}\left(x_{\beta_{2}} x_{\beta_{2}}^{\prime}\right) \cdot \rho_{Z Z^{\prime}}\left(z_{1} z_{1}^{\prime}\right) \cdot \rho_{Z Z^{\prime}}\left(z_{2} z_{2}^{\prime}\right) \cdot \rho_{\eta}\left(\frac{\Delta p_{1}}{p}\right) \cdot \rho_{\eta}\left(\frac{\Delta p_{2}}{p}\right) \cdot \rho_{S}\left(s_{1}\right) \cdot \rho_{S}\left(s_{2}\right),
$$

$d \tau$ is the infinitesimal element of the phase space $d x_{\beta_{1}} d x_{\beta_{1}}^{\prime} \ldots d s_{1} d s_{2}$.

At the time when the interaction takes place we have $s_{1}=s_{2}=s, z_{1}=z_{2}$ and $x_{1}=x_{2}$ where $x_{1,2}$ satisfies the equation (3.4).

The collision cross-section can be calculated by the following way. We approximately find for $\alpha<<1$ :

$$
2 \alpha \approx\left(\left(x_{2}^{\prime}-x_{1}^{\prime}\right)^{2}+\left(z_{1}^{\prime}-z_{2}^{\prime}\right)^{2}\right)^{1 / 2}
$$

Let's consider now the distribution of the scattering angle $\bar{\psi}$, which results from the Coulomb interaction of two charged particles considering the case of non-relativistic particles in the C.M. system one can use the Rutherford formula:

$$
\frac{d \bar{\sigma}(\bar{\psi})}{d \Omega}=\frac{1}{4}\left(\frac{Z^{2} e^{2}}{T}\right)^{2} \frac{1}{\sin ^{4}(\psi / 2)}, d \bar{\Omega}=\sin \psi \cdot d \bar{\psi} \cdot d \bar{\phi}
$$


$T$ is the kinetic energy of each colliding particle in the C.M. system.

Let $\bar{\psi}_{m}$ be the smallest scattering angle, its corresponding impact parameter $\bar{b}$ is given by the equation:

$$
\bar{b}=\frac{e^{2}}{2 T} \operatorname{ctg} \frac{\bar{\Psi}_{m}}{2} .
$$

Alternatively, equation (3.13) may be expressed as follows:

$$
\frac{d \bar{\sigma}(\bar{\psi})}{d \Omega}=\left(\frac{\frac{z^{2}}{A} r_{p}}{4 \beta^{2}}\right) \frac{1}{\sin ^{4}(\Psi / 2)},
$$

where $r_{p}=\frac{Z^{2} e^{2}}{A m_{p} c^{2}}$ is the ion classical radius, $m_{p}$ is the proton mass, $Z$ and $A$ are the ion charge and atomic numbers, $\bar{\beta} c$ is the particle velocity in the C.M. system and $\bar{\beta}<<1$ by our assumption. In a similar fashion one gets:

$$
\operatorname{tg} \bar{\Psi}_{m}=\frac{r_{i}}{2 \bar{\beta}^{2} \bar{b}}
$$

To evaluate $\bar{\beta}$ we express the two particle momenta difference, which is roughly equal to $2 m_{i} \bar{\beta} c$, in terms of their components in the C.M. reference frame. Then, using the Lorentz transformation for the momenta, we come back to the LRF. With all the calculations done we find in a first approximation:

$$
\bar{\beta} \approx \frac{1}{2} \beta \gamma\left(\frac{1}{\gamma^{2}}\left(\frac{\Delta p_{1}}{p}-\frac{\Delta p_{2}}{p}\right)^{2}+\left(x_{1}^{\prime}-x_{2}^{\prime}\right)^{2}+\left(z_{1}^{\prime}-z_{2}^{\prime}\right)^{2}\right)^{1 / 2}, \Delta P_{1,2}=\left(\vec{P}_{1,2}-\vec{P}_{0}\right)_{s}
$$

where $\beta c$ is the average particle velocity in the LRF and $\gamma$ is the associated Lorenz factor, $P$ corresponds to the particle with the velocity of $\beta c$. The integral required for calculation of formula (3.10) can be written as following:

$$
\text { Int }=\int_{\bar{\psi}_{m}}^{\pi} d \psi \int_{0}^{2 \pi} d \phi \frac{\delta \varepsilon}{\beta_{x}} \frac{d \bar{\sigma}}{d \Omega} \sin \Psi
$$

It is evaluated by substitution of the expressions given in equations $(3.1,3.2,3.8,3.15)$, which leads to the following result:

$$
\begin{gathered}
\text { Int }=\frac{\pi r_{o}^{2}}{16 \beta^{4}} \int_{\Psi_{m}}^{\pi} d \psi \frac{\sin \psi}{\sin ^{4}(\psi / 2)}\left\{( 1 - \operatorname { c o s } \psi ) \left(\frac{2 x_{\beta}}{\beta_{x}}\left(\frac{\left(1+\alpha_{x}^{2}\right)}{\beta_{x}} D \gamma \xi+\alpha_{x}\left(D^{\prime} \gamma \xi-\theta\right)\right)+\right.\right. \\
\left.\quad+2 x_{\beta}^{\prime}\left(\frac{\breve{D} \gamma \xi}{\beta_{x}}-\theta\right)+\frac{\left(D^{2}+\breve{D}^{2}\right)}{\beta_{x}^{2}} \gamma^{2} \xi^{2}+\theta^{2}-\frac{2 D}{\beta_{x}} \gamma \xi \theta\right)+
\end{gathered}
$$




$$
\left.+\sin ^{2} \Psi\left(\frac{\left(D^{2}+\breve{D}^{2}\right)}{4 \beta_{x}^{2}} \gamma^{2}\left(\theta^{2}+\varsigma^{2}-2 \xi^{2}\right)+\frac{1}{4}\left(\xi^{2}+\varsigma^{2}-2 \theta^{2}\right)+\frac{3 \breve{D}}{2 \beta_{x}} \gamma \xi \theta\right)\right\} .
$$

The definite integrals over the scattering angle $\Psi$ can be calculated analytically:

$$
\int_{\Psi_{m}}^{\pi} \frac{\sin \bar{\psi}(1-\cos \bar{\psi})}{\sin ^{4}(\Psi / 2)} d \bar{\psi}=-\left.4 \ln \left(\frac{1-\cos \bar{\psi}}{2}\right)\right|_{\bar{\psi}_{m}} ^{\pi}=4 \ln \left(1+\frac{4 \bar{\beta}^{4} \bar{b}^{2}}{r_{i}^{2}}\right),
$$

since $1-\cos \psi=2 \operatorname{tg}^{2}(\psi / 2) /\left(1+\operatorname{tg}^{2}(\psi / 2)\right)$ and $\operatorname{tg} \psi_{\mathrm{m}}$ with the Formula (3.16).

In a similar way one finds:

$$
\int_{\psi_{m}}^{\pi} \frac{\sin ^{3} \psi}{\sin ^{4}(\psi / 2)} d \psi=-\left.8\left(\cos ^{2}(\psi / 2)+\ln \left(\frac{1-\cos \psi}{2}\right)\right)\right|_{\bar{\psi}_{m}} ^{\pi}=8 \ln \left(1+\frac{4 \bar{\beta}^{4} \bar{b}^{2}}{r_{0}^{2}}\right)
$$

under the assumption $4 \bar{\beta}^{4} b^{2} / r_{0}^{2}>>1$.

Taking advantage of these results one gets:

$$
\begin{gathered}
\text { Int }=\frac{\pi \cdot r_{i}^{2}}{8 \bar{\beta}^{4}}\left\{\frac{4 x_{\beta}}{\beta_{x}}\left(\frac{\left(1+\alpha_{x}^{2}\right)}{\beta_{x}} D \gamma \xi+\alpha_{x}\left(D^{\prime} \gamma \xi-\theta\right)\right)+4 x_{\beta}^{\prime}\left(\frac{\breve{D}}{\beta_{x}} \gamma \xi-\theta\right)+\xi^{2}+\varsigma^{2}+\right. \\
\left.+\frac{\left(D^{2}+\breve{D}^{2}\right)}{\beta_{x}^{2}} \gamma^{2}\left(\theta^{2}+\varsigma^{2}\right)+\frac{2 \bar{D}}{\beta_{x}} \gamma \xi \theta\right\} \ln \left(1+\frac{4 \bar{\beta}^{4} \bar{b}^{2}}{r_{i}^{2}}\right) .
\end{gathered}
$$

Let us perform the substitutions into (3.22) (in full accordance with Formula (3.2)):

$$
x_{\beta_{1,2}}=x_{\beta} \pm \frac{D \gamma \cdot \xi}{2}, x_{\beta_{1,2}}^{\prime}=x_{\beta}^{\prime} \pm \frac{\theta-D^{\prime} \gamma \cdot \xi}{2}, z_{1,2}^{\prime}=z^{\prime} \pm \frac{\xi}{2}, \quad\left(\frac{\Delta p}{p}\right)_{1,2}=\eta \pm \frac{\gamma \cdot \xi}{2}
$$

(Note that $\eta$ is not here the usual eta function $-(\Delta f / f) /(\Delta p / p)$ ).

Hence, by virtue of the theorem on functions of random variables, the joint probability density law $P$ expressed in terms of these new variables takes the following form:

$$
P\left(x_{\beta}, x_{\beta}^{\prime}, z, z^{\prime}, \eta, s, \xi, \theta, \zeta\right)=|J| \cdot P\left(x_{\beta_{1}}, x_{\beta_{1}}^{\prime}, x_{\beta_{2}}^{\prime}, z_{1}, z_{1}^{\prime}, z_{2}^{\prime}, \frac{\Delta p_{1}}{p}, \frac{\Delta p_{2}}{p}, s\right)
$$

and $|J|=\gamma$, where $\mathrm{J}$ is the Jacobian of the transformation (3.23).

In accordance with calculations carried out earlier (eq.3.22), we obtain: 


$$
\begin{aligned}
& \frac{d}{d \bar{t}} \cdot \frac{\langle\varepsilon}{\beta_{X}}=\frac{\pi}{4} \cdot c r_{0}^{2} \gamma \int P\left\{\frac{4 x_{\beta_{1}}}{\beta_{X}}\left(\frac{\left(1+\alpha_{X}^{2}\right)}{\beta_{X}} \cdot D \cdot \gamma \cdot \xi+\alpha_{X}\left(D^{\prime} \cdot \gamma \cdot \xi-\theta\right)\right)+\right. \\
& \left.4 x_{\beta_{1}}^{\prime}\left(D^{\prime} \gamma \cdot \xi-\theta\right)+\xi^{2}+\zeta^{2}-2 \theta^{2}+\frac{\left.\left(D^{2}\right)+\widetilde{D}^{2}\right)}{\beta_{x}^{2}} \gamma^{2}\left(\zeta^{2}+\theta^{2}-2 \xi^{2}\right)+\frac{6 \gamma}{\beta_{X}} \widetilde{D} \xi \cdot \theta\right\} . \\
& \cdot \ln \left(1+\frac{4 \bar{\beta}^{4} \bar{b}^{2}}{r_{0}^{2}}\right) \frac{d \tau}{\bar{\beta}^{3}}
\end{aligned}
$$

where $d \tau=d x_{\beta} d x_{\beta}^{\prime} d z d z^{\prime} \cdot d \eta \cdot d s \cdot d \xi \cdot d \theta \cdot d \zeta$.

Clearly, $P$ is symmetrical with respect to $\xi, \theta$ and $\zeta$. Therefore, the integral vanishes for the linear terms in $\xi, \theta, \zeta$ of the integrand. Consequently, we are left with:

$$
\begin{aligned}
& \frac{d}{d t} \cdot \frac{\langle\varepsilon\rangle}{\beta_{X}}=\frac{\pi}{4} \cdot c r_{0}^{2} \gamma \int P\left\{\xi^{2}+\zeta^{2}-2 \theta^{2}+\frac{\left.\left(D^{2}\right)+\widetilde{D}^{2}\right)}{\beta_{x}^{2}} \gamma^{2}\left(\zeta^{2}+\theta^{2}-2 \xi^{2}\right)+\frac{6 \gamma}{\beta_{X}} \widetilde{D} \xi \cdot \theta\right\} . \\
& \cdot \ln \left(1+\frac{4 \beta^{4} b^{2}}{r_{0}^{2}}\right) \frac{d \tau}{\beta^{3}}
\end{aligned}
$$

This formula is obtained without an assumption about particle distribution, hence this integral can be calculated for an arbitrary distribution function. All the analytical models described below are based on an assumption that all betatron amplitudes and angles as well as the momentum deviations obey Gaussian distribution, while the particle distribution in the longitudinal direction is assumed to be uniform if the beam is unbunched, otherwise it is assumed to be Gaussian as well.

\section{Analytical models of IBS}

\subsection{Piwinski model}

In his model [1], A. Piwinski derives the formulae for the variations of the mean radial and vertical emittances and the mean momentum spread per unit time due to a scattering event, neglecting the derivative of the beta and dispersion functions with respect to the longitudinal beam axis. For the smoothed focusing approximation only the mean values of the lattice functions are used and they are determined as follows:

$$
\beta_{h, v}=\frac{R}{Q_{h, v}}, D=\frac{R}{Q_{h}^{2}}, \alpha_{h, v}=0, D^{\prime}=0
$$

where $R$ is the ring mean radius, $Q_{h, v}$ are horizontal and vertical betatron tunes. In this model the growth rates are calculated in accordance with the following expressions:

$$
\begin{gathered}
\frac{1}{\tau_{p}}=\frac{1}{2 \sigma_{p}^{2}} \frac{d \sigma_{p}^{2}}{d t}=n A \frac{\sigma_{h}^{2}}{\sigma_{p}^{2}} f(a, b, c), \\
\frac{1}{\tau_{x}}=\frac{1}{2 \sigma_{x \beta}^{2}} \frac{d \sigma_{x \beta}^{2}}{d t}=A\left[f\left(\frac{1}{a}, \frac{b}{a}, \frac{c}{a}\right)+\frac{D^{2} \sigma_{p}^{2}}{\sigma_{x \beta}^{2}} f(a, b, c)\right],
\end{gathered}
$$




$$
\frac{1}{\tau_{z}}=\frac{1}{2 \sigma_{z}^{2}} \frac{d \sigma_{z}^{2}}{d t}=A f\left(\frac{1}{b}, \frac{a}{b}, \frac{c}{b}\right),
$$

where $n=1$ for a bunched beam and $n=2$ for an coasting beam,

$$
A=\frac{r_{i}^{2} c N_{b}}{64 \pi^{2} \sigma_{s} \sigma_{p} \sigma_{x \beta} \sigma_{z} \sigma_{x^{\prime}} \sigma_{z^{\prime}} \beta^{3} \gamma^{4}}-\text { for a bunched beam }
$$

For coasting beam one has to use the substitution $\frac{N_{b}}{\sigma_{s}} \rightarrow \frac{2 \sqrt{\pi} N}{C}$.

The standard deviations are determined here as follows:

$$
\sigma_{x_{\beta}, z}=\sqrt{\varepsilon_{x, z} \beta_{x, z}}, \sigma_{x_{\beta}^{\prime}, z^{\prime}}=\sqrt{\frac{\left(1+\alpha_{x, z}^{2}\right) \varepsilon_{x, z}}{\beta_{x, z}}},
$$

and $\sigma_{p}$ is the r.m.s. momentum spread. The function $f(a, b, c)$ is the following integral:

$$
f(a, b, c)=8 \pi^{2} \int_{0}^{1}\left[\ln \left(\frac{c^{2}}{2}\left(\frac{1}{\sqrt{p}}+\frac{1}{\sqrt{q}}\right)\right)-0.577\right]\left(1-3 x^{2}\right) \frac{d x}{\sqrt{p q}} .
$$

The following relations determine normalized parameters used in the Formula (4.5):

$$
\begin{aligned}
& a=\frac{\sigma_{h}}{\gamma \sigma_{x^{\prime}}}, b=\frac{\sigma_{h}}{\gamma \sigma_{z^{\prime}}}, c=\beta \sigma_{h} \sqrt{2 \frac{\rho_{\max }}{r_{i}}}, \frac{1}{\sigma_{h}^{2}}=\frac{1}{\sigma_{p}^{2}}+\frac{D^{2}}{\sigma_{x \beta}^{2}}, p=a^{2}+x^{2}\left(1-a^{2}\right), \\
& q=b^{2}+x^{2}\left(1-b^{2}\right)
\end{aligned}
$$

and the maximum impact parameter $\rho_{\max }$ is about 0.5 beam vertical size. Integral (4.5) is calculated numerically.

In the article [2] it was shown that the integrals (4.9) under assumptions (4.1) are reduced to the Piwinski integral (4.5) and Piwinski's model [1] is one of the simplifications of a more general model [2].

\subsection{Martini model}

In accordance with the Martini model [2] for Gaussian probability law the r.m.s parameter growth rates can be inserted into the calculations in the form of the corresponding characteristic times:

$$
\begin{gathered}
\frac{1}{\tau_{p}}=\left\langle\frac{n A}{2}\left(1-d^{2}\right) f_{1}\right\rangle \\
\frac{1}{\tau_{x^{\prime}}}=\left\langle\frac{A}{2}\left[f_{2}+\left(d^{2}+\widetilde{d}^{2}\right) f_{1}\right]\right. \\
\frac{1}{\tau_{z^{\prime}}}=\left\langle\frac{A}{2} f_{3}\right\rangle
\end{gathered}
$$


where angular brackets mean averaging over the ring circumference, $n=1$ for a bunched beam and $n=2$ for coasting beam,

$$
A=\frac{\sqrt{1+\alpha_{x}^{2}} \sqrt{1+\alpha_{z}^{2}} c r_{i}^{2} \lambda}{16 \pi \sqrt{\pi} \sigma_{x_{\beta}} \sigma_{x_{\beta}^{\prime}} \sigma_{z} \sigma_{z^{\prime}} \sigma_{p} \beta^{3} \gamma^{4}}
$$

$\lambda$ is the linear ion density:

$$
\lambda=\left\{\begin{array}{cl}
N / L, & \text { for coasting beam } \\
N_{b} /\left(2 \sqrt{\pi} \sigma_{s}\right), & \text { for a bunched beam }
\end{array}\right.
$$

The functions $f_{i}$ are integrals of the following form:

$$
f_{i}=k_{i} \int_{0}^{\infty} \int_{0}^{\pi} \int_{0}^{2 \pi} \sin \mu g_{i}(\mu, v) \exp [-D(\mu, v) z] \ln \left(1+z^{2}\right) d v d \mu d z
$$

with the coefficients $k_{1}=1 / c^{2}, k_{2}=a^{2} / c^{2}, k_{3}=b^{2} / c^{2}$, and

$$
\begin{gathered}
D(\mu, v)=\frac{\left\lfloor\sin ^{2} \mu \cos ^{2} v+\sin ^{2} \mu(a \sin v-\tilde{d} \cos v)^{2}+b^{2} \cos ^{2} \mu\right\rfloor}{c^{2}} \\
g_{1}(\mu, v)=1-3 \sin ^{2} \mu \cos ^{2} v \\
g_{2}(\mu, \nu)=1-3 \sin ^{2} \mu \sin ^{2} v+6 \tilde{d} \sin \mu \sin v \cos v / a \\
g_{3}(\mu, \nu)=1-3 \cos ^{2} \mu .
\end{gathered}
$$

The normalized parameters are to be calculated from the following expressions:

$$
a=\frac{\sigma_{y}}{\sigma_{x_{\beta}^{\prime}}} \sqrt{1+\alpha_{x}^{2}}, b=\frac{\sigma_{y}}{\sigma_{z^{\prime}}}, c=q \sigma_{y}, d=\frac{\sigma_{p}}{\sigma_{x}} D, \widetilde{d}=\frac{\sigma_{p}}{\sigma_{x}} \widetilde{D},
$$

where $\widetilde{D}=\alpha_{x} D+\beta_{x} D^{\prime}, \sigma_{x}^{2}=\sigma_{x_{\beta}}^{2}+D^{2} \sigma_{p}^{2}, \sigma_{y}=\frac{\sigma_{p} \sigma_{x_{\beta}}}{\gamma \sigma_{x}}$ and $q=2 \beta \gamma \sqrt{\frac{\sigma_{z}}{r_{i}}}$.

The integration over $z$ - variable can be performed numerically or one can use one of the simplified results. If the integration is approximately performed as:

$$
\int_{0}^{\infty} \exp [-D(\mu, v) z] \ln \left(1+z^{2}\right) d z \approx \frac{2 L_{C}}{D(\mu, v)}
$$

where $L_{C}$ is the Coulomb logarithm, which is approximately equal to 20 , this model is reduced to the Bjorken - Mtingwa model [3]. Other possible way is to use a few first terms of the integral expansion into series. For instance in accordance with the book by Abramowitz \& Stegun the integral: 


$$
\int_{0}^{\infty} \exp [-D(\mu, v) z] \ln \left(1+z^{2}\right) d z \approx
$$

at $D \geq 1$ can be approximated by the expression

$$
\approx \frac{2\left(\left(\left(\left(\frac{a_{4}}{D^{2}}+a_{3}\right) \frac{1}{D^{2}}+a_{2}\right) \frac{1}{D^{2}}+a_{1}\right) \frac{1}{D^{2}}+1\right)}{D^{3}\left(\left(\left(\left(\frac{b_{4}}{D^{2}}+b_{3}\right) \frac{1}{D^{2}}+b_{2}\right) \frac{1}{D^{2}}+b_{1}\right) \frac{1}{D^{2}}+1\right)},
$$

here $a_{1}=42.242855, a_{2}=302.757865, a_{3}=352.018498, a_{4}=21.821899, b_{1}=48.196927$, $b_{2}=482.485984, b_{3}=1114.978885, b_{4}=449.690326$.

And at $D<1$

$$
\begin{gathered}
\approx\left(\left(\left(\left(\left(a_{6} D+a_{5}\right) D+a_{4}\right) D+a_{3}\right) D+a_{2}\right) D+a_{1}\right) D+a_{0}+\frac{a_{m}}{D}+ \\
+\left(\left(\left(b_{5} D^{2}+b_{3}\right) D^{2}+b_{1}\right) D+\frac{b_{m}}{D}\right) \ln D
\end{gathered}
$$

here $a_{m}=-2.0 E, a_{0}=\pi, a_{1}=-1.5+E, a_{2}=-\pi / 6.0, a_{3}=(25.0-12.0 E) / 144.0, a_{4}=\pi / 120.0$, $a_{5}=(-49.0+20.0 * E) / 7200.0, a_{6}=-0.818 \pi / 5040.0, b_{m}=-2.0, b_{1}=1.0, b_{3}=-12.0 / 144.0$, $b_{5}=20.0 / 7200.0$ and $E=0.57721566490153286061$ is the Euler constant.

In any case, the integral is approximately proportional to $1 / D(\mu, v)$, and numerical integration over $\mu$ and $v$ - variables has to be performed accurately because the $D(\mu, v)$ value can be close to zero for some beam parameters. Correspondingly, number of the integration steps required to obtain good accuracy of the calculation depends on a specific task.

\subsection{Jie Wei model}

The simplified model proposed by Jie Wei [4] is based on the same model of the interparticle collisions as Piwinski and Martini models (section \{4.1\}). In the notation of [5] the characteristic times of r.m.s. beam parameter variation are expressed as follows $[4,5]$ :

$$
\left[\begin{array}{c}
\frac{1}{\sigma_{p}} \frac{d \sigma_{p}}{d t} \\
\frac{1}{\sigma_{x}} \frac{d \sigma_{x}}{d t} \\
\frac{1}{\sigma_{z}} \frac{d \sigma_{z}}{d t}
\end{array}\right]=\frac{A_{0}}{2} \int e^{-D z} \ln \left(1+C^{4} z^{2}\right)\left[\begin{array}{c}
n_{b}\left(1-d^{2}\right) g_{1} \\
a^{2} g_{2}+\left(d^{2}+\bar{d}^{2}\right) g_{1} \\
b^{2} g_{3}
\end{array}\right] \sin \theta d \theta d \varphi d z
$$


where $A_{0}=\frac{c r_{p}^{2} N Z^{4} \beta_{x} \beta_{z}}{32 \pi A^{2} \sigma_{x}^{2} \sigma_{z}^{2} \sigma_{p} \sigma_{s} \beta^{3} \gamma^{4}}$, for a bunched beam $N$ is the number of particles per bunch, $\sigma_{\mathrm{s}}$ is the rms bunch length. For coasting beam $N$ is the total number of particles and $\sigma_{s}=C / 2 \sqrt{\pi}$, where $\mathrm{C}$ is the ring circumference. $r_{p}=\frac{e^{2}}{m_{p} c^{2}}, d=\frac{D_{p} \sigma_{p}}{\sqrt{\sigma_{x}^{2}+D_{p}^{2} \sigma_{p}^{2}}}$ is the effective ratio of the "dispersion" amplitude $D_{p} \delta_{p}$ to the horizontal total one, $D_{p}$ is horizontal dispersion, $\bar{d}=\frac{\bar{D}_{p} d}{D_{p}}$, $\bar{D}_{p}=\alpha_{x} D_{p}+\beta_{x} D_{p}^{\prime}, a=\frac{\beta_{x} d}{D_{p} \gamma}, b=\frac{\beta_{z} \sigma_{x}}{\beta_{x} \sigma_{z}} a$,

$D=\cos ^{2} \theta+b^{2} \sin ^{2} \theta \sin ^{2} \varphi+(a \sin \theta \cos \varphi-\bar{d} \cos \theta)^{2}$,

$C=2 \beta \sigma_{p}\left[\sigma_{z}\left(1-d^{2}\right) / r_{p}\right]^{1 / 2}$,

$g_{1}=1-3 \cos ^{2} \theta$,

$g_{2}=\cos ^{2} \theta-2 \sin ^{2} \theta \cos ^{2} \varphi+\sin ^{2} \theta \sin ^{2} \varphi+6 \bar{d} \cos \theta \sin \theta \cos \varphi / a$,

$g_{3}=\cos ^{2} \theta+\sin ^{2} \theta \cos ^{2} \varphi-2 \sin ^{2} \theta \sin ^{2} \varphi$.

The actual growth rate, observed over a time which is long compared to the revolution period, is calculated by averaging over the circumference.

Apart from the different notation, the expression (4.17) coincides with the expression (4.6). The following simplifications of the (4.17) were proposed in [4]:

- the quantity $\ln \left(1+C^{4} z^{2}\right)$ can be substituted by a constant $2 L_{C}$, where $L_{C}$ is about 20 (this simplification is common for Jie Wei and Bjorken - Mtingwa models),

- for accelerator consisting of regular cells the variation in $D_{p} / \beta_{x}^{1 / 2}$ is small along the ring circumference, thus the terms including $\bar{D}_{p}$ and $\bar{d}$ can be neglected,

- for simplification of the integration over $\theta$ and $\varphi$ the $\sin ^{2} \varphi$ and $\cos ^{2} \varphi$ are replaced by their average value of $1 / 2$.

Then the formula (4.17) can be reduced to the following form:

$$
\left[\begin{array}{cc}
\frac{1}{\sigma_{p}} & \frac{d \sigma_{p}}{d t} \\
\frac{1}{\sigma_{x}} & \frac{d \sigma_{x}}{d t} \\
\frac{1}{\sigma_{z}} & \frac{d \sigma_{z}}{d t}
\end{array}\right]=4 \pi A_{0} L_{C} F(\chi)\left[\begin{array}{c}
n_{b}\left(1-d^{2}\right) \\
-a^{2} / 2+d^{2} \\
-b^{2} / 2
\end{array}\right]
$$

where $\chi=\left(a^{2}+b^{2}\right) / 2 \geq 0$

$$
F(\chi)=\frac{-3+(1+2 \chi) I(\chi)}{1-\chi}
$$




$$
I(\chi)= \begin{cases}\frac{1}{\sqrt{\chi(\chi-1)}} \operatorname{Arth} \sqrt{\frac{\chi-1}{\chi}}, \quad \chi \geq 1, \\ \frac{1}{\sqrt{\chi(1-\chi)}} \arctan \sqrt{\frac{1-\chi}{\chi}}, \quad \chi<1 .\end{cases}
$$

To calculate characteristic times one needs to average the expressions (4.18) over the ring circumference.

In terms of the normalized transverse emittance and longitudinal bunch area $S=\pi m_{0} c^{2} \gamma \sigma_{s} \sigma_{p} / \beta^{3} c A$ in phase space, Eq.(4.18) can be rewritten as

$$
\left[\begin{array}{c}
\frac{1}{\sigma_{p}} \frac{d \sigma_{p}}{d t} \\
\frac{1}{\sigma_{x}} \frac{d \sigma_{x}}{d t} \\
\frac{1}{\sigma_{y}} \frac{d \sigma_{y}}{d t}
\end{array}\right]=\frac{Z^{4} N}{A^{2}} \frac{r_{0}^{2} m_{0} c^{2} L_{c}}{8 \beta^{4} \gamma \varepsilon_{x} \varepsilon_{y} S} F(\chi)\left[\begin{array}{c}
n_{b}\left(1-d^{2}\right) \\
-a^{2} / 2+d^{2} \\
-b^{2} / 2
\end{array}\right]
$$

Except for the beam factors $\chi, \mathrm{d}$, $\mathrm{a}$ and $\mathrm{b}$ that depend on the ratio of the beam amplitudes in different dimension, the rates are linearly proportional to the density in the six-dimensional phase space, and are strongly dependent on the charge state of the particle.

The coupling between the horizontal and vertical motion averages the growth rates in the transverse dimension. If the motion is fully coupled within time periods much shorter than the IBS diffusion time, the average rates become:

$$
\left[\begin{array}{c}
\frac{1}{\sigma_{p}} \frac{d \sigma_{p}}{d t} \\
\frac{1}{\sigma_{x, y}} \frac{d \sigma_{x, y}}{d t}
\end{array}\right]=\frac{Z^{4} N}{A^{2}} \frac{r_{0}^{2} m_{0} c^{2} L_{c}}{8 \beta^{4} \gamma \varepsilon_{x} \varepsilon_{y} S} F(\chi)\left[\begin{array}{c}
n_{b}\left(1-d^{2}\right) \\
\left(-\chi+d^{2}\right) / 2
\end{array}\right]
$$

In a typically circular accelerator, the transition energy $\gamma_{\mathrm{T}}$ is approximately equal to the average value of $\beta_{x} / D_{p}$ in the regular cells. When the beam energy is high $\gamma>\gamma_{\mathrm{T}}$, the growth in horizontal direction results mostly from the variation of the betatron closed orbit during the exchange of the particle momentum $\left(a^{2}<<d^{2}\right)$. The growths in horizontal and longitudinal amplitudes are therefore proportional to each other.

In a typical storage ring like the Relativistic Heavy Ion Collider (RHIC) the beams are stored at energies much higher than the transition energy. Due to the coupling and injection conditions, the horizontal and vertical betatron amplitudes are about the same. The growth rates can be explicitly written from Eq. (4.18) using the expression for $F(\chi)$ : 


$$
\left[\begin{array}{c}
\frac{1}{\sigma_{p}} \frac{d \sigma_{p}}{d t} \\
\frac{1}{\sigma_{x, y}} \frac{d \sigma_{x, y}}{d t}
\end{array}\right]=\frac{Z^{4} N}{A^{2}} \frac{r_{0}^{2} m_{0} c^{2} L_{c}}{16 \beta^{4} \gamma_{T} \varepsilon_{x} \varepsilon_{y} S} F(\chi)\left[\begin{array}{c}
n_{b}\left(1-d^{2}\right) / d \\
d / n_{c}
\end{array}\right]
$$

Their dependence on the energy of the beam, which appears only in the form factor $\mathrm{d}$, is usually weak. After the initial stage of the storage, the asymptotic configuration

$$
n_{b} n_{c} \sigma_{x}^{2} \approx D_{p}^{2} \sigma_{p}^{2}, \quad \gamma>>\gamma_{T}
$$

will be approximately reached $\left(d \approx n_{b} n_{c} /\left(1+n_{b} n_{c}\right)\right)$.

\subsection{Bjorken - Mtingwa model}

To take into account dispersion in vertical plane as well as in horizontal the IBS growth rates are calculated in accordance with [7]:

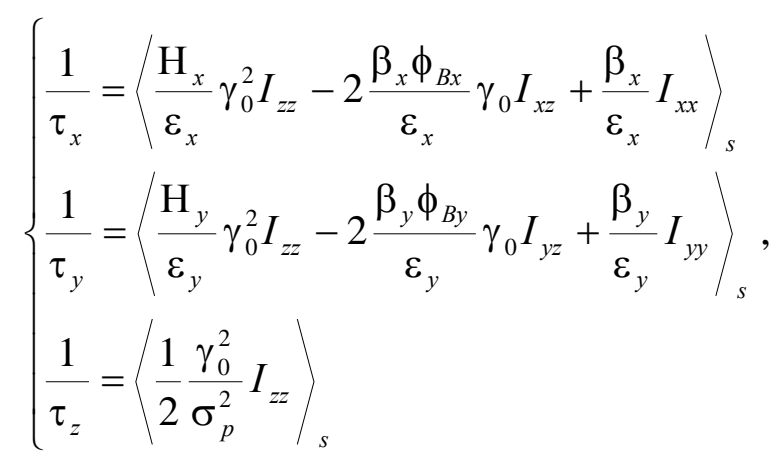

where $\phi_{B i}=D_{i}^{\prime}+\alpha_{i} D_{i} / \beta_{i}, \mathrm{H}_{i}=\beta_{i} D_{i}^{\prime 2}+2 \alpha_{i} D_{i} D_{i}^{\prime}+\gamma D_{i}^{2}$ and $\alpha_{i}, \beta_{i}, \gamma_{i}$ - lattice functions in the horizontal $(i=x)$ and vertical $(i=y)$ plane, $\varepsilon_{x, y}$ are the horizontal and vertical emittances, $\sigma_{p}-\mathrm{rms}$ momentum spread. Angular brackets mean averaging over the ring circumference. At zero vertical dispersion these formulae coincide with original Bjorken-Mtingwa theory [3]. The diffusion coefficients $I_{i j}$ are calculated in each position of the ring by numerical evaluation of the following integrals

$$
I_{i j}=A \int_{0}^{\infty} d \lambda \frac{\lambda^{1 / 2}}{\sqrt{\operatorname{det} \Lambda}}\left(\delta_{i j} \operatorname{Tr} \Lambda^{-1}-3 \Lambda_{i j}^{-1}\right),
$$

where the matrix $\Lambda=I \lambda+L, I-$ unit matrix, and matrix $L$ is calculated via beam rms parameters and ring lattice functions in accordance with: 


$$
L=\left[\begin{array}{ccc}
\frac{\beta_{x}}{\varepsilon_{x}} & 0 & -\gamma_{0} \frac{\beta_{x} \phi_{B x}}{\varepsilon_{x}} \\
0 & \frac{\beta_{y}}{\varepsilon_{y}} & -\gamma_{0} \frac{\beta_{y} \phi_{B y}}{\varepsilon_{y}} \\
-\gamma_{0} \frac{\beta_{x} \phi_{B x}}{\varepsilon_{x}} & -\gamma_{0} \frac{\beta_{y} \phi_{B y}}{\varepsilon_{y}} & \frac{\gamma_{0}^{2} \mathrm{H}_{x}}{\varepsilon_{x}}+\frac{\gamma_{0}^{2} \mathrm{H}_{y}}{\varepsilon_{y}}+\frac{\gamma_{0}^{2}}{\sigma_{p}^{2}}
\end{array}\right] .
$$

The IBS constant $A$ is determined as in other IBS models:

$$
A=\frac{c r_{i}^{2} N L_{c}}{8 \pi \beta^{3} \gamma_{0}^{2} \varepsilon_{x} \varepsilon_{y} \sigma_{p} \sigma_{s}} .
$$

Here $\beta$ and $\gamma_{0}$ are the Lorenz parameters, $r_{i}$ is the ion classical radius, $\mathrm{N}$ is the ion number, $L_{\mathrm{c}}$ is the Coulomb logarithm, which is introduced as an input parameter.

\subsection{Gas Relaxation model}

Diffusion coefficients for a spatially uniform gas of density $\mathrm{n}$ and isotropic Maxwellian distribution are well known. The rate of such diffusion can be written as

$$
\tau^{-1}=\frac{4 \pi n(Z e)^{4}}{m^{2}} \frac{\Lambda}{\Delta^{3}}
$$

where $\Delta$ is the one-dimensional rms velocity and $\Lambda$ is the Coulomb logarithm. The heating rate is determined by the 6-D phase-space density of the gas $\mu=n /\left(m^{3} \Delta^{3}\right)$, where $\mathrm{n}$ is the spatial density. Similarly, the diffusion coefficients can be derived for a longitudinally collapsed velocity distribution:

$$
f(\vec{r}, \vec{v})=\frac{n}{\pi \sqrt{\pi} \Delta_{\perp}^{2} \Delta_{\|}} e^{-\left(v_{x}^{2}+v_{y}^{2}\right) / \Delta_{\perp}^{2}} e^{-v_{z}^{2} /\left(2 \Delta_{\|}^{2}\right)},
$$

with $\Delta_{\|}<<\Delta_{\perp}$. The resulting coefficient is [8]:

$$
D_{z z}=\frac{4 \pi n(Z e)^{4}}{m^{2}} \frac{\Lambda}{\Delta_{\perp}}\left[\sqrt{\pi} e^{\left.-u^{2}\right) /\left(2 \Delta_{\perp}^{2}\right)} I_{0}\left(\frac{u^{2}}{2 \Delta_{\perp}^{2}}\right)\right]
$$

Due to a slow decrease of function in the square brackets with its argument, one can replace expression in the square brackets by 1 . As a result, one gets

$$
D_{z z} \approx \frac{4 \pi n(Z e)^{4}}{m^{2}} \frac{\Lambda}{\Delta_{\perp}}
$$

The growth rate in the longitudinal directions is then given by 


$$
\tau_{\|}^{-1}=\frac{1}{\Delta_{\|}^{2}} \frac{d \vec{v}_{z}^{2}}{d t}=4 \pi m(Z e)^{4} \mu \Lambda \frac{\Delta_{\perp}}{\Delta_{\|}},
$$

where the 6-D phase-space density $\mu$ is defined as $\mu=n /\left(m^{3} \Delta_{\perp}^{2} \Delta_{\|}\right)$.

One can rewrite diffusion coefficient

$$
D_{z z} \approx \frac{4 \pi n(Z e)^{4}}{m^{2}} \frac{\Lambda}{\Delta_{\perp}}
$$

in terms of beam parameters. In the laboratory system it becomes [9]:

$$
D_{z z}=\frac{2 r_{i}^{2} c N_{i} \Lambda}{\beta^{3} \gamma^{3} \varepsilon_{\perp}^{3 / 2} \beta_{\perp}^{1 / 2} C}
$$

where for the 6-D phase-space density we used $\mu=n /\left(m^{3} \Delta_{\perp}^{2} \Delta_{\|}\right)=N / V_{6}$, with the 6-D volume being $V_{6}=(2 \pi)^{3}(\beta \gamma)^{3} m^{3} \varepsilon_{x} \varepsilon_{y} \varepsilon_{z}$. Here, the formula is written for coasting beam with $\mathrm{C}$ being the ring circumference. Note that the normalization factor in velocity space was already taken into account in the derivation of (4.34). One then needs only the 3-D spatial contribution to the normalization coefficient to get (4.35). Expression given in (4.35) was used in the original version of the SimCool code to represent diffusion rate due to the IBS. The longitudinal heating rate for a bunched beam with $\mathrm{C}$ being replaced by $2 \sqrt{\pi} \sigma_{s}$ (following the standard definition in the IBS theory, which corresponds to $92 \%$ of a Gaussian beam in longitudinal direction) is then [9]:

$$
\tau_{\|}^{-1}=\frac{1}{\sigma_{p}^{2}} \frac{d \sigma_{p}^{2}}{d t}=\frac{r_{i}^{2} c N_{i} \Lambda}{8 \beta^{3} \gamma^{3} \varepsilon_{x}^{3 / 2}\left(\beta_{\perp}^{1 / 2}\right) \sqrt{\pi / 2 \sigma_{s} \sigma_{p}^{2}}}
$$

The transverse growth rate can be simply expressed through the longitudinal growth rate as

$$
\tau_{\perp}^{-1}=\frac{\sigma_{p}^{2}}{\varepsilon_{x}}\left(\frac{D_{x}^{2}+\left(D_{x}^{\prime} \beta_{x}+\alpha_{x} D_{x}\right)^{2}}{\beta_{x}}\right) \tau_{\|}^{-1},
$$

where $\langle>$ is an average value of the ring lattice. For the RHIC lattice the contribution of the term with dispersion derivatives and alpha-function is small (smooth lattice) and can be neglected so that only $\left\langle D_{x}^{2} / \beta_{x}\right\rangle$ may be considered. The transverse heating rate based on (4.37) were implemented in the version of the SimCool code updated by the BNL group and in BETACOOL code. They were benchmarked vs standard IBS formalism for the RHIC parameters.

\subsubsection{High energy approach}

For high energies at RHIC (when approximation of flattened velocity distribution in the beam rest frame becomes valid), simple gas-relaxation formula gives reasonable approximation for description of the longitudinal heating rate, compared to the involved IBS treatment with complicated dependence on the lattice parameters [9].

The standard IBS formulas can be simplified for the high-energy case. For example, BjorkenMtingwa (B-M) model can be approximated for a round beam at high-energy as 


$$
\tau_{\|}^{-1}=\frac{r_{i}^{2} c N_{i} \Lambda}{8 \beta^{3} \gamma^{3} \varepsilon_{x}^{3 / 2}\left(\beta_{\perp}^{1 / 2}\right\rangle \sigma_{s} \sigma_{p}^{2}}
$$

The high-energy approximation of B-M agrees very well with the one obtained using the GasRelaxation model in Eq. (4.36). This confirms that the main effect in IBS diffusion at high energy is determined by a degree of a collapse of velocity distribution in the beam moving frame of reference. Treatment of the IBS in such a case is then extremely simplified. To describe applicability region of the high-energy approximation one typically introduces parameter $g_{f}$ which describes a degree of a collapse of ion velocity distribution, and is defined as $g_{f}=\left(\frac{\Delta_{\|}}{\Delta_{\perp}}\right)^{2}=\frac{\left.\beta_{\perp}\right) \sigma_{p}^{2}}{\gamma^{2} \varepsilon_{\perp}}$.

For typical parameters of Au ions at RHIC store energy $g_{f}$ is in the range 0.1-0.2 which justifies the use of high-energy approximation treatment of the IBS for simple estimates.

\subsection{Calculations of IBS in the case of transverse coupling}

In the case when transverse motion is completely coupled within one revolution over the ring the invariant change after interparticle collision has to be calculated using bilinear forms of the lattice in the collision position instead of formulae (3.8) and (3.9). Theory of the IBS for coupled motion was done by Piwinski in [6]. When transverse coupling in the ring has small value the IBS rates can be calculated using formulae for uncoupled motion. At RHIC parameters IBS growth times are rather long - a few tens of minutes. At such a long time the transverse emittances are connected together even at a small coupling. If the motion is completely coupled within time period much shorter than IBS diffusion time it is possible to use average value of the transverse rates for both degrees of freedom:

$$
\frac{1}{\tau_{x, z, \text { coupled }}}=\frac{1}{2}\left(\frac{1}{\tau_{x}}+\frac{1}{\tau_{z}}\right)_{\text {uncoupled }}
$$

Such a manner of the coupling insertion into calculation is used in our code for all the models of IBS. For instance, in Jie Wei model the average rates become:

$$
\left[\begin{array}{cc}
\frac{1}{\sigma_{p}} & \frac{d \sigma_{p}}{d t} \\
\frac{1}{\sigma_{x, z}} & \frac{d \sigma_{x, z}}{d t}
\end{array}\right]=4 \pi A_{0} L_{C} F(\chi)\left[\begin{array}{c}
n_{b}\left(1-d^{2}\right) \\
\left(-\chi+d^{2}\right) / 2
\end{array}\right] .
$$

\section{Kick of the ion momentum components due to IBS}

\subsection{Mean growth rates}

In the case of IBS calculation using mean growth rates, the mean growth rates are calculated in accordance with one of the analytical model developed in BETACOOL and the ring structure 
loaded from output MAD file. When the growth rates are known one can calculate mean square of the scattering angle taking into account multiplication factor. The mean square angle after one revolution in the ring can be calculated under assumption that the alpha function and dispersion in the position of calculation are zero. In this case initial beam emittance is equal to:

$$
\varepsilon_{x}=\sqrt{\left.(x-\langle x\rangle)^{2} /\left(x^{\prime}-\left\langle x^{\prime}\right\rangle\right)^{2}\right\rangle}
$$

After the scattering of all the particles on the randomly distributed angle $\theta$ the new emittance value is

$$
\varepsilon_{x, \text { new }}=\sqrt{\left.\left.(x-\langle x\rangle)^{2}\right\rangle\left(x^{\prime}-\left\langle x^{\prime}\right\rangle+\theta\right)^{2}\right\rangle} \text {. }
$$

New r.m.s. angular spread value can be calculated as follows

$$
\left\langle\left(x^{\prime}-\left\langle x^{\prime}\right\rangle+\theta\right)^{2}\right\rangle=\left\langle\left(x^{\prime}-\left\langle x^{\prime}\right\rangle\right)^{2}\right\rangle+2\left(x^{\prime}-\left\langle x^{\prime}\right\rangle\right) \theta+\left\langle\theta^{2}\right\rangle
$$

The second is equal zero because $\mathrm{x}^{\prime}$ and $\theta$ are independent random values, therefore the emittance can be expressed as:

$$
\varepsilon_{x, \text { new }}=\varepsilon_{x, 0} \sqrt{1+\frac{\left\langle\theta^{2}\right\rangle}{\left\langle\left(x^{\prime}-\left\langle x^{\prime}\right\rangle\right)^{2}\right\rangle}}
$$

Assuming that the square scattering angle is less than the beam r.m.s. angular spread one can expand this expression into Tailor series with accuracy to the first term. It gives for the scattering angle such an equality:

$$
\left\langle\theta^{2}\right\rangle=2 \frac{\Delta \varepsilon_{x}}{\varepsilon_{x, 0} \beta_{x}}
$$

Using the rate value definition one can writes for the scattering angle:

$$
\left\langle\theta^{2}\right\rangle=2 \frac{\varepsilon}{\beta \tau} T_{r e v},
$$

where $\beta$ is the beta function in the point of the particle array generation, $\tau$ is characteristic growth time in corresponding degree of freedom. Here the angular deviation of the particle trajectory means relative momentum components: $\theta_{x, y}=\frac{p_{x, y}}{p}, \theta_{s}=\frac{\Delta p}{p}$. After $N_{t u r n}$ revolutions in the ring the square of the scattering angle is equal to the sum of the square angles at each revolution:

$$
\left\langle\theta^{2}\right\rangle=2 \frac{\varepsilon}{\beta} \frac{T_{r e v}}{\tau} N_{t u r n} .
$$

The variation of the particle trajectory angular deviation is calculated in accordance with 


$$
\Delta \theta=\sqrt{\theta^{2} \xi \xi}
$$

where $\xi$ is the random value with Gaussian distribution at unit dispersion.

\subsection{Detailed calculation of the momentum kick}

\subsubsection{Burov's model}

In the case of a detailed calculation of the growth rates the algorithm is similar to the previous case. However, the characteristic times are calculated for each particle individually. For this purpose one needs to know not only the r.m.s. parameters of the particle array distribution function, but the amplitudes of the betatron and synchrotron oscillations of the particle. The inverse growth times for each particle can be calculated by numerical evaluation of the scattering integrals. Under some additional assumption the integration can be performed analytically for one degree of freedom. For instance, in the frame of Burov's model of detailed IBS calculation, the longitudinal IBS rate is presented as [10]:

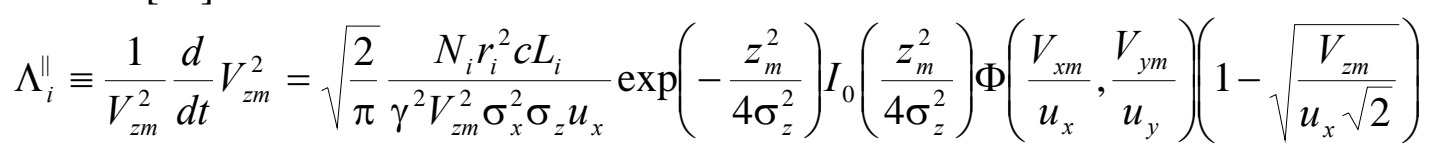

Here $u_{x}$ are rms velocities in PRF, $s_{x, y}$ - rms beam sizes. $z m$ - amplitude of the ion oscillations over longitudinal co-ordinate, $V_{x m, y m}$ are the amplitudes of oscillations over transverse velocities, $I_{0}-$ modified Bessel function. The form-factor $\Phi$ is given by the expression:

$$
\Phi(x, y) \equiv \frac{2}{\pi} \exp \left(-\frac{x^{2}+y^{2}}{2}\right) \iint_{0} \frac{d \xi d \eta}{\sqrt{\xi^{2}+\eta^{2}}} \exp \left(-\frac{\xi^{2}+\eta^{2}}{2}\right) I_{0}(\xi x) I_{0}(\eta y)
$$

Horizontal IBS rate relates to the longitudinal rate as:

$$
\Lambda_{i}^{x} / \Lambda_{i}^{\|}=\frac{\overline{D^{2}+\left(D^{\prime} \beta_{x}+\alpha_{x} D\right)^{2}}}{x_{m}^{2}} V_{z m}^{2},
$$

where the bar stands for averaging over the ring.

The formula (5.8) is derived on the basis of plasma relaxation model and does not contain averaging over the ring lattice parameters. The formula is based under assumption of Gaussian distribution of the ions flattened in velocity space. To test the model at the same model of IBS process at initial step of the simulations in the program the following combination of r.m.s. rates calculation and detailed formula is used. Square of the particle scattering angle is calculated as

$$
\left\langle\theta^{2}\right\rangle=\left\langle\theta_{\text {mean }}^{2}\right\rangle F\left(V_{z m}, V_{x m}, V_{y m}\right),
$$

where $\left\langle\theta_{\text {mean }}^{2}\right\rangle$ is calculated using analytical model of IBS in accordance with expression (2.24). The form factor $F$ is calculated accordingly to (5.8) as 


$$
F\left(V_{z m}, V_{x m}, V_{y m}\right)=\exp \left(-\frac{z_{m}^{2}}{4 \sigma_{z}^{2}}\right) I_{0}\left(\frac{z_{m}^{2}}{4 \sigma_{z}^{2}}\right) \Phi\left(\frac{V_{x m}}{u_{x}}, \frac{V_{y m}}{u_{y}}\right)\left(1-\sqrt{\frac{V_{z m}}{u_{x} \sqrt{2}}}\right) \times C .
$$

Normalization constant $C$ is calculated numerically to satisfy the condition: variation of r.m.s. beam parameters with time has no depends on the way of IBS calculation - mean or detailed.

\subsubsection{Core-tail model}

Model Beam algorithm can simulate beam dynamics with the distribution of model particles.

Standard models of IBS assume that the beam has Gaussian distribution. In the case of Model Beam algorithm in BETACOOL the particles can have an arbitrary distribution.

To address this problem, the "core-tail" approach was introduced [11]. In this approach, the parameters of the core and tails of the distribution could be defined based on either FWHM model or by fitting two Gaussians. The corresponding methods in BETACOOL are called "FWHM" and "bi-Gaussian", respectively.

When the distribution function can be accurately presented as a sum of two Gaussian functions for core and tail particles, the "bi-Gaussian" model provides an accurate fit. In this case

$$
f\left(x_{i}\right)=f^{\text {tail }}\left(x_{i}\right)+f^{\text {core }}\left(x_{i}\right)=a_{i}^{\text {tail }} \exp \left[-\frac{1}{2}\left(\frac{x_{i}}{\sigma_{i}^{\text {tail }}}\right)^{2}\right]+a_{i}^{\text {core }} \exp \left[-\frac{1}{2}\left(\frac{x_{i}}{\sigma_{i}^{\text {core }}}\right)^{2}\right],
$$

where $a^{\text {tail }}, a^{\text {core }}, \sigma^{\text {tail }}, \sigma^{\text {core }}-$ amplitudes and widths of beam profile, $i$ - corresponds to degrees of freedom: 1 - horizontal, 2 - vertical, 3 - longitudinal. Powell method [12] is used in the code to find of Gaussian function parameters. This method minimizes the deviation between the model beam profile and Bi-Gaussian distribution:

$$
\sum_{i=1}^{N}\left[y_{i}-f\left(x_{i}\right)\right]^{2} \rightarrow \text { Minimum }
$$

where $\left(x_{i}, y_{i}\right)$ - points of the beam profile distribution, $N$ is the total particle number. RMS parameters of ion beam $E_{i}^{r m s}$ (emittances and momentum spread) are calculated in the suggestion that distribution of model particles has Gaussian shape. Beam parameters and particle number for Bi-Gaussian distributions are:

$$
E_{i}^{\text {tail }}=E_{i}^{\text {rms }}\left(\sigma_{i}^{\text {tail }}\right)^{2}, \quad E_{i}^{\text {core }}=E_{i}^{\text {rms }}\left(\sigma_{i}^{\text {core }}\right)^{2}, \quad N_{i}^{\text {core }}=N \times\left[1+\frac{\sigma_{i}^{\text {tail }}}{\sigma_{i}^{\text {core }}} \cdot \frac{a_{i}^{\text {tail }}}{a_{i}^{\text {core }}}\right]^{-1}, \quad N_{i}^{\text {tail }}=1-N_{i}^{\text {core }}
$$

Heating growth rates $\tau_{i}^{\text {tail }}\left(E_{i}^{\text {tail }}, N\right)$ and $\tau_{i}^{\text {core }}\left(E_{i}^{\text {core }}, N\right)$ of Bi-Gaussian distributions are calculates with standard procedure as described above in section $\{4\}$ on the base of choosen IBS model for Gaussian distribution. Model particles in the tail get the kick with standard procedure as described in Section 5.1 using of tail heating growth rates.

$$
\Delta \theta_{1,2}^{\text {tail }}=\sqrt{2 \frac{\varepsilon_{1,2}^{\text {tail }}}{\beta_{1,2}} T_{1,2}^{\text {tail }}} N_{\text {turn }} \times \xi, \quad \Delta \theta_{3}^{\text {tail }}=\sqrt{\left(\frac{\Delta p^{\text {tail }}}{p}\right)^{2} \frac{T_{\text {rev }}}{\tau_{3}^{\text {tail }}} N_{\text {turn }} \times \xi,}
$$


where $\varepsilon_{1,2}$ - horizontal and vertical emittances, $\beta_{1,2}$ - horizontal and vertical beta-functions, $(\Delta \mathrm{p} / \mathrm{p})-$ momentum spread, $T_{\text {rev }}$ - revolution period, $N_{\text {turn }}$ - number of turns, $\xi$ - the random value with Gaussian distribution at unit dispersion. Particles in the core get the kick from core and tail heating rates:

$$
\begin{gathered}
\frac{1}{\tau_{i}^{\text {sum }}}=\frac{1}{\tau_{i}^{\text {core }}}\left(\frac{N_{i}^{\text {core }}}{N}\right)^{2}+\frac{1}{\tau_{i}^{\text {tail }}}\left(\frac{N_{i}^{\text {tail }}}{N}\right)^{2} \frac{E_{i}^{\text {tail }}}{E_{i}^{\text {core }}} . \\
\Delta \theta_{1,2}^{\text {core }}=\sqrt{2 \frac{\varepsilon_{1,2}^{\text {core }}}{\beta_{1,2}} \frac{T_{\text {rev }}}{\tau_{1,2}^{\text {sum }}} N_{\text {turn }} \times \xi} \\
\Delta \theta_{3}^{\text {core }}=\sqrt{\left(\frac{\Delta p^{\text {core }}}{p}\right)^{2} \frac{T_{\text {rev }}}{\tau_{3}^{\text {sum }}} N_{\text {turn }} \times \xi}
\end{gathered}
$$

\subsubsection{Parzen's model for rms rates of bi-Gaussian distribution}

The model for IBS growth rates calculation proposed by G. Parzen [13] is based on presentation of the ion distribution function as a sum of two Gaussian distributions. $N f(x, p)$ gives the number of particles in $d^{3} x d^{3} p$, where $N$ is the number of particles in a bunch. For a bi-Gaussian distribution, $f(x, p)$ is given by

$$
f(x, p)=\frac{N_{c}}{N} \frac{1}{\Gamma_{c}} \exp \left[-S_{c}(x, p)\right]+\frac{N_{t}}{N} \frac{1}{\Gamma_{t}} \exp \left[-S_{t}(x, p)\right]
$$

Here $N_{c}$ and $N_{t}$ are the particle number in the first (corresponding tu the core of total distribution) and second Gaussian (describing the tail of the total distribution). $\Gamma_{c, t}=\int \exp \left(-S_{c, t}(x, p)\right) d^{3} x d^{3} p$ are the corresponding normalization factors,

$$
S=S_{x}+S_{y}+S_{s}
$$

where

$$
S_{i}=\frac{I_{i}}{\varepsilon_{i}}, i=x, y, s
$$

is the ratio of the particle invariant of the motion $I$ to 2 -sigma emittance $\varepsilon$ in the corresponding plane.

Because of $N_{c}+N_{t}=N$ the distribution (5.18) is described by 7 independent parameters: $\varepsilon_{x, c}, \varepsilon_{y, c}, \varepsilon_{s, c}, \varepsilon_{x, t}, \varepsilon_{y, t}, \varepsilon_{s, t}$ and $N_{c}$. To find these parameters for arbitrary array of particles one needs to solve 7-D optimization problem, which looks unrealistic. To avoid this difficulty the 7-D optimization problem was replaced by solution of 3 independent 3-D optimization problem. For each degree of freedom from smoothed beam profile the program calculates rms emittances for core and tail of distribution function using Powel method [12]. As a result of the calculations one has a set of 9 parameters: 


$$
\begin{aligned}
& \varepsilon_{x, c}, \varepsilon_{x, t}, N_{x, c}, \\
& \varepsilon_{y, c}, \varepsilon_{y, t}, N_{y, c}, \\
& \varepsilon_{s, c}, \varepsilon_{s, t}, N_{s, c},
\end{aligned}
$$

the emittances are used as corresponding parameters of Bi-Gaussian distribution, and intensity of core Gaussian is calculated as:

$$
N_{c}=\frac{N_{x, c}+N_{y, c}+N_{s, c}}{3} .
$$

Thereafter the program calculates additional four parameters, required for evaluation of growth rates for total beam:

$$
\varepsilon_{x, c t}=\frac{\varepsilon_{x, c} \varepsilon_{x, t}}{\varepsilon_{x, c}+\varepsilon_{x, t}}, \varepsilon_{y, c t}=\frac{\varepsilon_{y, c} \varepsilon_{y, t}}{\varepsilon_{y, c}+\varepsilon_{y, t}}, \varepsilon_{s, c t}=\frac{\varepsilon_{s, c} \varepsilon_{s, t}}{\varepsilon_{s, c}+\varepsilon_{s, t}} \text { and } N_{c t}=\frac{N_{c} N_{t}}{N^{2}}
$$

The diffusion coefficients for core and tail Gaussians are calculated in accordance with BjorkenMtingwa model:

$$
\begin{aligned}
I_{i j, c} & =A_{c} \int_{0}^{\infty} d \lambda \frac{\lambda^{1 / 2}}{\sqrt{\operatorname{det} \Lambda_{c}}}\left(\delta_{i j} \operatorname{Tr} \Lambda_{c}^{-1}-3 \Lambda_{i j, c}^{-1}\right), \\
I_{i j, t} & =A_{t} \int_{0}^{\infty} d \lambda \frac{\lambda^{1 / 2}}{\sqrt{\operatorname{det} \Lambda_{t}}}\left(\delta_{i j} \operatorname{Tr} \Lambda_{t}^{-1}-3 \Lambda_{i j, t}^{-1}\right), \\
I_{i j, c t} & =A_{c t} \int_{0}^{\infty} d \lambda \frac{\lambda^{1 / 2}}{\sqrt{\operatorname{det} \Lambda_{c t}}}\left(\delta_{i j} \operatorname{Tr} \Lambda_{c t}^{-1}-3 \Lambda_{i j, c t}^{-1}\right),
\end{aligned}
$$

where matrixes $\Lambda_{c}, \Lambda_{t}, \Lambda_{c t}$ are calculated in accordance with (4.27) with substitution of corresponding emittances. The IBS constants are calculated as usual:

$$
A_{i}=\frac{c r_{i}^{2} N_{i} L_{c}}{8 \pi \beta^{3} \gamma_{0}^{2} \varepsilon_{x, i} \varepsilon_{y, i} \sigma_{p, i} \sigma_{s, i}} .
$$

Diffusion coefficients for the total beam are calculated as:

$$
I_{i j}=I_{i j, c} \frac{N_{c}}{N}+I_{i j, t} \frac{N_{t}}{N}+2 I_{i j, c t} .
$$

This procedure is repeated in each optics element of the ring, and resulting heating rates are averaged over the ring circumference.

The described algorithm does not coincide exactly with the original paper [13]. However, it uses the same final formula (5.28) and is based on the same modification of the Bjorken-Mtingwa model, which simplifies benchmarking of the model. 


\section{References}

[1] A. Piwinski, Proc. $9^{\text {th }}$ Int. Conf. on High Energy Accelerators, p. 105 (1974).

[2] M. Martini "Intrabeam scattering in the ACOOL-AA machines", CERN PS/84-9 AA (1984).

[3] J.D. Bjorken, S.K. Mtingwa, Particle Accelerators, Vol. 13, p.115 (1983).

[4] J. Wei, Proceedings of 1993 PAC, p.3651 (1993).

[5] G. Parzen, Nuclear Instr. Meth. A256, 231 (1987); Proc. 1988 EPAC, Rome, p.281.

[6] A. Piwinski, Proc CERN Accelerator School, 92-01, p.405 (1992).

[7] M. Venturini, Proceedings of 2001 PAC, Chicago, p. 2961 (2001).

[8] A. Sorensen, CERN Acc. School (1987).

[9] A. Fedotov, BNL Tech Note C-AD/AP/168 (2004).

[10] A. Burov. FERMILAB-TM-2213 (2003).

[11] A. Fedotov et al., Proc. of 2005 PAC (Knoxville, TN, 2005), p. 4263.

[12] W. Press, S. Teukolsky, W. Vetterling, B. Flannery. Numerical Recipes in C. The Art of

Scientific Computing (Second Edition). Cambridge University Press (1992)

[13] G. Parzen, BNL Tech Note C-AD/AP/169 (2004). 